\section{Oral Communications with posters}

Published online: 11 August 2006

\section{HEADACHES: PATHOGENETIC ASPECTS}

\section{EFFECT OF NITROGLYCERIN ON TEMPORAL SUMMATION THRESHOLD OF NOCICEPTIVE FLEXION REFLEX IN MIGRAINE PATIENTS}

${ }^{1}$ M. Serrao, ${ }^{2} A$. Perrotta, ${ }^{2} G$. Sandrini, ${ }^{3} P$. Rossi, ${ }^{2} G$. Sances, ${ }^{2}$ E. Guaschino, ${ }^{1}$ F. Pierelli, ${ }^{2,4}$ G. Nappi

${ }^{1}$ UO Riabilitazione, Polo Pontino-ICOT, University of Rome "La Sapienza", Latina, Italy; ${ }^{2}$ University Centre for Adaptive Disorders and Headache, IRCCS “C. Mondino" Institute of Neurology Foundation, University of Pavia, Pavia, Italy; ${ }^{3}$ Headache Clinic, INI Grottaferrata, Grottaferrata, Rome, Italy; ${ }^{4}$ Department of Neurology and Otolaryngology, University of Rome "La Sapienza", Rome, Italy; e-mail: armando.perrotta@mondino.it

Background and aims In migraineurs, nitroglycerin (NTG) induces severe delayed headache, resembling spontaneous migraine attacks. The temporal summation threshold (TST) of the nociceptive flexion reflex (NFR) is considered an objective method that offers the potential to examine pain processing in the central nervous system. The aim of the present study was to evaluate the NFR-TST during NTGinduced migraine.

Methods Twenty-nine patients (19 females and 10 males, mean age $36.2 \pm 7.67$, range $18-55$ years), suffering from migraine without aura (1.1 ICHD-II, 2004) were selected. Headache was induced by sublingual administration of $0.9 \mathrm{mg}$ of NTG. NFR-TST and the subjective painful sensation were measured before and 30, 60, 120 and $240 \mathrm{~min}$ after drug administration.

Results All patients exhibited a significant $(p<0.01)$ reduction of the NFR-TST 120 min after drug administration. In particular, a sub-group of patients experiencing severe attack showed a significant $(p<0.01)$ reduction of NFR-TST at both 60 and 120 minutes.

Conclusions NTG appears to support a reliable experimental model of migraine, based on the neuronal effects on the integrative-nociceptive structures. The NFR-TST facilitation during NTG-induced attack reflects an extracephalic generalized hypersensitivity of pain pathways during migraine attack that provides the susceptibility for triggering migraine attack and probably its persistence.

CAN HIGH-FREOUENCY rTMS RESTORE NORMAL INTRACORTICAL EXCITABILITY IN MIGRAINE WITH AURA? EFFECTS OF PRIMING STIMULATION ON 1 HZ rTMS

${ }^{I}$ F. Brighina, ${ }^{1}$ G. Giglia, ${ }^{1}$ A. Palermo, ${ }^{2}$ A. Aloisio, ${ }^{1}$ V. Saia, ${ }^{1}$ B. Fierro ${ }^{I}$ Department of Neurology, Ophthalmology, Otorhinolaryngology and Psychiatry, University of Palermo, Palermo, Italy; ${ }^{2}$ Neurology Unit, ARNAS Civic Hospital, Palermo, Italy; e-mail: fbrighina@unipa.it

Introduction We showed that motor intracortical inhibition (ICI) is reduced in patients affected by migraine with aura. Consequently, $1 \mathrm{~Hz}$ rTMS-induced paradoxical effects increase motor intracortical facilitation (ICF) in these patients. In conditions of enhanced excitability due to reduced inhibition, high frequency rTMS acts by potentiating intracortical inhibition $[1,2]$

On this basis, we used high frequency priming stimulation of the motor cortex to enhance intracortical inhibition and reverse paradoxical facilitation induced by $1 \mathrm{~Hz}$ rTMS in migraine with aura patients. To differentiate the possible different effects on ICF or ICI, we applied the double-pulse TMS technique that allows evaluation of the intracortical circuits.
Methods Seven patients with migraine with aura and six controls underwent a paired-pulse TMS paradigm to evaluate ICI and ICF of the motor cortex before and after a conditioning high frequency rTMS followed by $1 \mathrm{~Hz}$ rTMS train. Patients were examined interictally at least $48 \mathrm{~h}$ before or after an attack. The hot-spot for the right abductor pollicis brevis (ABP) was checked by mean of a figure-of-eight coil and motor threshold (MT) on this recorded point. ICI and ICF were assessed by means of a conditioning stimulus (CS) at $80 \%$ MT followed by a test stimulus (TS) at $120 \%$ MT, with two different interstimulus intervals: inhibitory: $2 \mathrm{msec}$ and facilitatory $10 \mathrm{msec}$. Priming rTMS (900 stimuli at $10 \mathrm{~Hz}$ frequency, in trains of 50 stimuli separated by $45 \mathrm{~s}$ intervals) was followed by a single 15 -min-long train (900 stimuli) at $1 \mathrm{~Hz}$ rTMS. rTMS was delivered at $90 \%$ of MT over the hotspot. Motor evoked potential (MEP) amplitude of TS post-rTMS was expressed as percentage of baseline MEP. Amplitude of MEPs recorded at 2 and $10 \mathrm{~ms}$ ISIs before and after rTMS were expressed as percentage of change from the respective mean TS alone.

Results At baseline, ICI was significantly lower in migraineurs with respect to controls. In healthy controls, MEP amplitude and ICF were significantly reduced by priming $1 \mathrm{~Hz}$ treatment with respect to baseline values. In contrast, the priming stimulation significantly increased ICI in migraineurs, leaving quite unchanged MEP amplitude and ICF values with respect to baseline.

Conclusions In migraineurs, rTMS-priming potentiates intracortical inhibition and reverses the paradoxical increase in ICF observed with $1 \mathrm{~Hz}$ rTMS [1]. These findings strengthen the hypothesis of reduced inhibition in migraine with aura and might open perspectives for new treatment strategies.

References

1. Fierro B, Brighina F, Vitello G et al (2005) Modulatory effects of low- and high-frequence repetitive transcranial magnetic stimulation on visual cortex of healthy subjects undergoing light deprivation. J Physiol 565:659-665

2. Siebner HR, Mentschel C, Auer C et al (2000) Repetitive transcranial magnetic stimulation causes a short-term increase in the duration of the cortical silent period in patients with Parkinson's disease. Neurosci Lett 284:147-150

\section{EFFECTS OF REMOTE CUTANEOUS PAIN ON TRIGEMINAL LASER EVOKED POTENTIALS IN NORMAL SUBJECTS AND MIGRAINE PATIENTS}

M. de Tommaso, M. Sardaro, C. Pecoraro, O. Difruscolo, P. Livrea

Neuropathophysiology Unit of Chronic Pain, Department of Neurological and Psychiatric Sciences, University of Bari, Bari, Italy; e-mail:m.detommaso@neurol.uniba.it

Introduction In a previous study a different modulation of trigeminal laser evoked potentials (LEPs) was detected in migraine patients vs controls during topical application of capsaicin on the supraorbital skin. The inhibiting central effect exerted in controls by capsaicin on trigeminal LEPs was less evident in migraine, suggesting a disturbed pattern of pain modulation at the cortical level, which may subtend the onset and persistence of migraine [1].

Objective The aim of the present study was to detect the functional changes of trigeminal LEPs during topical application of capsaicin over a remote site of the body in migraine patients outside the attacks $v s$. non migraine healthy controls.

Methods Ten patients suffering from migraine without aura (ICHD-II, 2004), free from pain and symptomatic drugs for at least 72 hours and not taking preventive treatment for migraine were selected for the study. Eight age- and sex-matched healthy controls were also examined. LEPs were recorded by 31 scalp electrodes, referred to the nasion, stimulating the dorsum of the right hand and the right supraorbital zone in basal conditions and 30 minutes after application of $3 \mathrm{~mL}$ of $3 \%$ capsaicin in a cream base (Teofarma), which was applied topically on the entire surface of the dorsum of the left hand, within an area 
of at least $6 \mathrm{~cm}^{2}$. The subjective pain induced by both laser stimulus and capsaicin was rated by a $0-100$ visual analogue scale (VAS).

Results In normal subjects, the N2-P2 vertex complex showed $32.3 \% \pm 3.4 \%$ rate of amplitude reduction at the trigeminal level and $30.2 \% \pm 7.8 \%$ at the hand level, caused by remote capsaicin application. In migraine, the rate of N2-P2 amplitude reduction at the hand level was similar to controls $(31.3 \pm 9.8 ; \mathrm{F}=0.67$, n.s), while it was significantly lower at the face level $(2.1 \% \pm 4.5 \% \mathrm{~F}=16.5, p<0.001)$. The N1 amplitude was not significantly modified by capsaicin, when both the face and the hand were stimulated. N2-P2 amplitude modifications were reversed after capsaicin removal in both patients and controls. Discussion The N2-P2 amplitude reduction induced in normal subjects at both the hand and face levels could be due to a diffuse noxious inhibitory control and to the distraction induced on LEPs by concurrent capsaicin-induced pain. Both the pain-inhibiting mechanisms appeared inadequate in reducing experimental trigeminal pain in migraine patients.

Conclusions In migraine patients trigeminal pain is not inhibited by the anti-nociceptive endogenous control system.

Reference

1. de Tommaso M, Losito L, Difruscolo O et al (2005) Changes in cortical processing of pain in chronic migraine. Headache 45:1208-1218

\section{SLEEP STUDIES IN PRIMARY HEADACHES}

C. Vollono, A. Capuano, D. Ferraro, D. Mei, G. Della Marca, G. Di Trapani

Headache Centre, "A. Gemelli" Hospital, Rome, Italy; e-mail: catello.vollono@rm.unicatt.it

Introduction Primary headaches have an intimate relationship with sleep. Although strictly sleep-related headaches, such as hypoxemiarelated headache, cluster headache $(\mathrm{CH})$ and hypnic headache $(\mathrm{HH})$ are less prevalent and more easily recognized, other primary headaches are often impacted to some degree by sleep.

Objective The aim of the study was to describe the results of sleep studies performed in different forms of primary headache and their pathophysiological correlates.

Methods Patients underwent a full-night laboratory nocturnal videopolysomnography following adaptation. Patients slept in a sound-proof room. A wrist actigraphic study was also performed in patients with $\mathrm{HH}$ and $\mathrm{CH}$.

Results Hypnic Headache: The most relevant finding regarding sleep structure was the quantitative reduction of rapid eye movement (REM) sleep. Actigraphic recordings showed that the index of motor activity was consistently increased following the start of effective drug treatment. This might constitute further support for the hypothesis of hypoarousal as a pathogenic mechanism for $\mathrm{HH}$.

Cluster Headache: No modifications of polysomnographic arousal parameters across the recordings were observed in the acute phase, when compared with the interictal phase, except for the arousal index in REM sleep. At a microstructural level, the arousal index calculated in REM was much lower in the nights in which attacks occurred. Actigraphic recordings revealed, moreover, that the patient's sleepwake schedule was irregular during the $\mathrm{CH}$ period and progressively modified, becoming more regular after remission. All the observed modifications are consistent with an abnormal function of the posterior hypothalamus.

Sleep-related migraine: Evaluation of the pattern of arousal from sleep in a group of patients affected by sleep-related migraine showed a lower continuous airway pressure (CAP) rate in non REM sleep and, in particular, a lower number of A1 phases (low-frequency, high-amplitude EEG bursts) compared with controls. Migraineurs also showed a lower index of high-frequency EEG arousals during REM sleep. Reduction in the CAP rate indicates a lower level of arousal fluctuation in non REM sleep. The reduced arousal index in REM sleep suggests a dysfunction in neural structures involved in both the control of REM sleep and the pathophysiology of migraine, such as the hypothalamus and the brainstem.

Conclusions We believe that sleep studies, including both macro- and microstrucural analysis of sleep and actigraphy, may be useful in clarifying the pathogenic mechanisms of primary headaches.

\section{DESTABILIZING CONDITIONS IN MIGRAINE PATIENTS EVALUATED BY COMPUTERIZED STATIC STABILOMETRY} ${ }^{I}$ C. Rossi, ${ }^{l}$ P. Sarchielli, ${ }^{I}$ F. Coppola, ${ }^{l}$ P. Calabresi, ${ }^{2}$ M. Faralli, ${ }^{2}$ G. Ricci, ${ }^{2}$ A. Frenguelli

${ }^{1}$ Headache Centre, Department of Medical Surgical Specialties and Public Health, ${ }^{2}$ Otolaryngology Clinic, University of Perugia, Perugia, Italy; e-mail: mentira@libero.it

Objective The aim of this study was to assess the presence of destabilizing inputs that, by acting on vestibulospinal pathways, are able to induce postural changes and imbalance conditions in migraine patients during the interictal period, by computerized static stabilometry.

Material and methods Twenty patients were studied (14 F and $6 \mathrm{M}$; mean age 32.6 years) affected by migraine without aura (MO) according to ICHD-II, 2004 criteria and were compared with 35 controls. The stabilometric parameters considered were: statokinesigram surface (S) in eyes open (EO) and closed (EC) conditions, EC with head retroflexion (ECR), EC condition with occlusal bite, and optokinetic stimulation $(\mathrm{OKN})$. Regarding the $\mathrm{OKN}$ condition, we used two different kinds of stimulation: the "look" (active) type, in which the patient must follow with the eyes the visual stimulation running through the monitor, and the "stare" (passive) type, in which the patient must look at a fixed point on the monitor and not the visual stimulation crossing the monitor.

Results We found a significant statistical difference $(p<0.045)$ between patients and controls in only one stabilometric condition, the OKN stimulation. In particular, the OKN "stare" stimulation was more destabilizing for migraine patients than for controls, inducing conditions of imbalance.

Conclusions In MO patients, the alterations appear under optokinetic stimulation and support a control impairment in involuntary oculomotility of central origin. This is suggested by recent stabilometric findings of our group [1].

Reference

1. Rossi C, Alberti A, Sarchielli P et al (2005) Balance disorders in headache patients: evaluation by computerized static stabilometry. Acta Neurol Scand 111:407-413

\section{AUTONOMIC DYSFUNCTION IN MIGRAINE WITH AURA}

${ }^{1}$ A. Fiorentini, ${ }^{1}$ L. Tubani, ${ }^{l} R$. Valente, ${ }^{2}$ E. Celletti, ${ }^{l} L$. Baratta, ${ }^{1}$ A. Perciaccante, ${ }^{2} M$. Granata

${ }^{1}$ Cardiology and ${ }^{2}$ Headache Centre, Department of Clinical Medicine, University of Rome "La Sapienza", Rome, Italy; e-mail: massimo.granata@uniromal.it

Introduction Some studies suggested that migraine is associated with an impaired activity of the autonomic nervous system [1]. It is known that the subject with migraine has a reduction in R-R intervals and a reduction in the increase of the heart rate during "handgrip" in comparison with the control group [2].

Objective The objective of our study was to analyse in subjects with migraine with aura the heart rate variability (HRV), evaluated in time and frequency domains by means of ECG holter during 24 hours.

Patients and methods We recruited subjects with migraine with aura during headache-free periods, without treatment. The control group consisted of age- and sex-matched healthy control subjects. Autonomic nervous system function was evaluated by HRV analysis during 24-hour ECG recording, SDNN (millisec, total autonomic 
activity), RMS-SD (millisec parasympathetic component), low frequency (LF, modulated by the sympathetic system) and high frequency (HF, mediated by the parasympathetic system) were calculated. The day was divided into two periods: diurnal (7-12 a.m.) and nocturnal (0-6 a.m.).

Results Autonomic function measures: SDNN day (millisec): migraine 146.16 \pm 28.41 , control 175.92 $\pm 30.13, p=0.38$; SDNN night: migraine $116.26 \pm 22.23$, control 121.98 $\pm 26.59, \quad p=0.63$; RMS-SD day: $121.98 \pm 26.59$, control $121.98 \pm 26.59, p<0.001$; RSM-SD night: migraine $48.19 \pm 15.28$, control $48.19 \pm 15.28, p=0.75$; LF day (normalized unit): migraine $61.20 \pm 2.27$, control $69.90 \pm 8.34, p=0.003$; HF day: migraine 38.80 \pm 2.27 , control 22.95 $\pm 7.31, p=0.002$; LF night: migraine $55.13 \pm 10.72$, control $34.92 \pm 5.99, p=0.03$; HF night: migraine $44.95 \pm 10.69$, control 58.62 $\pm 6.77, p=0.03$.

Conclusions In subjects with migraine, the data showed the increase of parasympathetic activity during the day and the predominance of the sympathetic component at night with loss of circadian rhythms. It remains unclear if the alteration of the autonomic nervous system is a risk factor for migraine, or instead, is a consequence of the pathology.

References

1. Peroutka SJ (2004) Migraine: a chronic sympathetic nervous system disorder. Headache 44:53-64

2. Benjelloun H, Birouk N, Slaoui I et al (2005) Autonomic profile of patients with migraine. Neurophysiol Clin 35:127-134

\section{HIGH-FREQUENCY OSCILLATION SOMATOSENSORY EVOKED POTENTIALS: EVIDENCE OF A REDUCED THALA- MO-CORTICAL ACTIVATION IN MEDICATION-OVERUSE HEADACHE}

${ }^{1}$ G. Coppola, ${ }^{2}$ A. Ambrosini, ${ }^{3} J$. Schoenen, ${ }^{4}$ F. Pierelli

${ }^{I}$ Research Foundation for the Study of Ophthalmology "G. Bietti", Rome, Italy; ${ }^{2}$ IRCCS-Neuromed, Pozzilli, Isernia, Italy; ${ }^{3}$ Headache Research Unit, University Departments of Neurology and Neuroanatomy, University of Liège CHR Citadelle, Belgium; ${ }^{4}$ University of Rome "La Sapienza", Polo Pontino, I.C.O.T., Latina, Italy; e-mail: gianluca.coppola@uniroma1.it

Background Recently, studies using FDG-PET techniques have reported hypoactivation of nuclei belonging to the "pain network" in patients with medication-overuse headache $(\mathrm{MOH})$. In particular, it has been shown that the ventral posteromedial thalamic nuclei were hypometabolic in migraineurs suffering from $\mathrm{MOH}$ [1]. Since the high-frequency oscillations (HFOs) embedded in the common somatosensory evoked potentials (SSEPs) recording reflect spike activity in thalamo-cortical cholinergic fibers (early HFOs phase) and cortical inhibitory GABA-ergic interneurons (late HFOs phase), we studied a group of $\mathrm{MOH}$ patients with this neurophysiological approach.

Materials and methods Right median nerve SSEPs were recorded from the contralateral parietal area in 9 patients affected by medication-overuse headache (MOH, ICHD-II 8.2) and in 9 healthy volunteers (HV). Digital off-line filtration (band-pass between 450 and 750 $\mathrm{Hz}$ ) was employed in order to extract the high frequency $(600 \mathrm{~Hz})$ oscillations embedded in the broad-band SSEPs.

Results The amplitudes and latencies of conventional broad-band SSEPs showed no between-group differences. After applying the digital filter, we found a significant decrease of maximal amplitude $(p=0.030)$ and area under the rectified curve $(p=0.038)$ of the early SSEP HFO component in patients compared to HV. By contrast, no difference was found for the late SSEP HFO component.

Discussion These results show thalamo-cortical hypoactivity in patients with $\mathrm{MOH}$. This is in line with the previous FDG-PET study showing thalamic hypometabolism. The absence of SSEP HFO late component changes suggests normal intracortical inhibition at the level of the somatosensory cortex in this patient group.

\section{Reference}

1. Fumal A, Laureys S, Di Clemente L et al (2006) Orbitofrontal cortex involvement in chronic analgesic-overuse headache evolving from episodic migraine. Brain 129:543-550, Epub 2005 Dec 5

\section{INCREASED CEREBROSPINAL FLUID LEVELS OF NERVE GROWTH FACTOR AND BRAIN-DERIVED NEUROTROPHIC FACTOR ARE NOT SPECIFIC FOR CHRONIC MIGRAINE: COMPARISON WITH FIBROMYALGIA}

M.L. Mancini, C. Rossi, F. Coppola, A. Baldi, I. Corbelli, P. Calabresi, P. Sarchielli

Headache Centre, Neurologic Clinic, Department of Medical Surgical Specialties and Public Health, Univeristy of Perugia, Perugia, Italy; $e$ mail:mlmancini@tautech.it

Background Experimental findings from animal pain models support the role of nerve growth factor (NGF) and brain-derived neurotrophic factor (BDNF) as putative candidates intervening in the pathogenesis of chronic pain. Few studies have been carried out to establish their role in maintaining the pain states in humans and their involvement in chronic migraine (CM) and fibromyalgia $[1,2]$.

Objective The present study was aimed at investigating cerebrospinal fluid (CSF) levels of NGF and glutamate measured by sensitive immunoassay and by HPLC respectively in CM patients and patients affected by primary fibromyalgia syndrome (PFMS), comparing values with those of 20 age-matched control subjects.

Material and methods Twenty consecutive patients suffering from $\mathrm{CM}$ according to ICHD-II classification and attending the Headache Centre of the Neurologic Clinic of the University of Perugia were admitted to the study.

A further patient group included 20 patients affected by PFMS. Diagnosis of PFMS was made according to the American College of Rheumatology Criteria (1990).

All patients were admitted to the Neurologic Clinic to undergo lumbar puncture. Control CSF specimens were obtained from 20 age-matched subjects who underwent lumbar puncture for diagnostic purposes. In all subjects CSF and blood tests excluded CNS or systemic diseases. All control subjects were drug-free for at least two months and none of them were taking any medication at the time of CSF sampling nor had a personal or family history of migraine nor suffered from tension-type headache. None of the above controls developed post-lumbar puncture headache.

Results Significantly higher levels of both neurotrophins were found both in patients with CM $(p<0.0001, p<0.0005)$ and PFMS $(p<0.001$ and $p<0.001)$ compared with control subjects, without significant differences between the two patient groups. Both patient groups had significantly higher CSF levels of glutamate than controls $(p<0.001$ and $p<0.003$, respectively). A significantly positive correlation emerged between CSF values of BDNF and those of NGF $(r=0.61, p<0.001$; $r=0.53, p<0.01)$ and glutamate $(r=0.44, p<0.02 ; r=0.51, p<0.01)$ in both CM and PFMS patients, respectively.

Conclusions These findings suggest the possibility of an NGF-mediated up-regulation of BDNF in central sites involved in long-term sensitization, which plays a key role in persistent, chronic painful conditions, such as CM and fibromyalgia. NGF might indirectly exert its effect through enhancement of glutamatergic transmission via BDNF. The above mechanisms could account for sustained central sensitization in both chronic pain states but cannot be considered specific for just one of these two disorders. They may rather be considered expression of the pathogenic mechanisms underlying chronic pain per se.

\section{References}

1. Sarchielli P, Alberti A, Floridi A, Gallai V (2001) Levels of nerve growth factor in cerebrospinal fluid of chronic daily headache patients. Neurology 57:132-134

2. Giovengo SL, Russell IJ, Larson AA (1999) Increased concentrations of nerve growth factor in cerebrospinal fluid of patients with fibromyalgia. J Rheumatol 26:1564-1569 


\section{ENDOCANNABINOIDS IN PLATELETS OF MEDICATION- OVERUSE HEADACHE PATIENTS AND CHRONIC MIGRAINE PATIENTS: CORRELATION WITH SEROTONIN LEVELS}

${ }^{1}$ C. Rossi, ${ }^{2}$ L.A. Pini, ${ }^{1}$ P. Calabresi, ${ }^{1}$ P. Sarchielli

${ }^{1}$ Neurologic Clinic, Department of Medical and Surgical Specialties and Public Health, University of Perugia, Perugia, Italy; ${ }^{2}$ Institute of Clinical Pharmacology, University of Modena and Reggio Emilia, Modena, Italy; e-mail: mentira@libero.it

Background Based on experimental evidence of the antinociceptive action of endocannabinoids [1], we hypothesized a dysfunction of this system in medication-overuse headache $(\mathrm{MOH})$ and chronic migraine $(\mathrm{CM})$ Objective To test this hypothesis, we determined the levels of the endogenous cannabinoid 2-arachidonoylglycerol (2-AG) and arachidonoylethanolamide (anandamide, AEA) in platelets of 15 patients affected by $\mathrm{MOH}$ according to ICHD-II and 15 patients with CM without medication overuse, as well in 15 age-matched control subjects. We also investigated the intra-platelet levels of serotonin.

Methods Endogenous cannabinoids were purified from platelet pellets by high-performance liquid chromatography (HPLC), and quantified by isotope dilution gas-chromatography/mass-spectrometry. Serotonin levels were also measured by HPLC.

Results In both patients and controls levels of 2-AG were about 20fold greater than that of AEA. 2-AG and AEA levels were significantly lower in medication overuse and CM patients than in controls $(p<0.02$ and $p<0.04$, respectively), without significant differences between the two groups. Serotonin levels were strongly reduced in both patient groups $(p<0.001$ and $p<0.002$, respectively) and were significantly correlated with $2-\mathrm{AG}$ and serotonin levels $(r=0.48$, $p<0.01$ and $r=0.46, p<0.02$ ).

Conclusions The finding of reduced 2-AG and AEA in platelets correlated with reduced serotonin levels may reflect an imbalance in the endocannabinoid system, which occurs in parallel with serotonergic dysfunction in these two chronic head pain conditions. The parallelism in the failure of these two systems is in line with experimental findings of mutual interaction of 2-AG and 5-HT in modulating different signalling pathways, which can also be relevant for pain control Variations in activity of AEA transporter and AEA hydrolase in peripheral platelets, as recently shown in female but not male migraineurs [2], remain to be established in $\mathrm{MOH}$ and $\mathrm{CM}$.

This study has been partially supported by the Lega Italiana Cefalalgici.

References

1. Walker JM, Hohmann AG (2005) Cannabinoid mechanisms of pain suppression. Handb Exp Pharmacol (168):509-554

2. Cupini LM, Bari M, Battista N et al (2006) Biochemical changes in endocannabinoid system are expressed in platelets of female but not male migraineurs. Cephalalgia 26:277-281

\section{THE WOLFRAMIN HIS611ARG POLYMORPHISM INFLU- ENCES MEDICATION-OVERUSE HEADACHE}

${ }^{1,2}$ C. Di Lorenzo, ${ }^{3}$ G. Di Lorenzo, ${ }^{1}$ C. Rengo, ${ }^{4}$ G. Sances, ${ }^{4}$ N. Ghiotto, ${ }^{4}$ E. Guaschino, ${ }^{5}$ F.M. Santorelli, ${ }^{1}$ G.S. Grieco, ${ }^{3}$ A. Siracusano, ${ }^{2}$ F. Pierelli, ${ }^{4,6}$ G. Nappi, ${ }^{1,2}$ C. Casali

${ }^{I}$ Molecular Genetics Laboratory, IRCCS Neurological Institute "C. Mondino" Foundation, IRCCS "San Raffaele”, Rome, Italy, ${ }^{2}$ Department of Neurology and ORL, and University Centre for Adaptive Disorders and Headache (UCADH), University of Rome "La Sapienza", Polo Pontino, Latina, Italy; ${ }^{3}$ Psychiatric Clinic, Department of Neuroscience, University of Rome "Tor Vergata", Rome, Italy; ${ }^{4}$ Headache Unit, and University Centre for Adaptive Disorders and Headache (UCADH), IRCCS Neurological Institute "C. Mondino" Foundation, Pavia, Italy; 5 Molecular Medicine, IRCCS "Bambino Gesù", Rome, Italy; ${ }^{6}$ Department of Neurology and Otolaryngology, University of Roma "La Sapienza”, Rome, Italy;e-mail: cherub@inwind.it
Introduction The wolframin gene (WFS1) is mentioned as being possibly responsible for a heterogeneous combination of psychiatric disorders; in a number of studies it has been suggested that common variants in WFS1 are associated with psychiatric illnesses. For instance, homozygous carriers of the His611Arg polymorphism (R/R genotype) are at increased risk of attempted suicide and generally score higher than non-R/R individuals on both novelty seeking (NS), impulsivity and depression scales. It is well known that psychiatric comorbidity is widely represented in medication-overuse headache $(\mathrm{MOH})$. In the present study we investigated the wolframin His611 Arg polymorphism in a sample of $\mathrm{MOH}$ patients who submitted to psychiatric, psychometric and clinical assessment.

Materials and methods Fifty-two consecutive, unrelated $\mathrm{MOH}$ patients (42 women and 10 men) were recruited. Mean age was $46.37 \pm 11.35$ years; mean analgesic-drug consumption was $44.81 \pm 28.24$ doses/month. All the patients were interviewed using the Structured Clinical Interview for DSM-IV Axis I Disorders (SCID-I), to obtain a categorical psychiatric diagnosis, and completed the Beck Depression Inventory (BDI), a self-reported questionnaire for a dimensional assessment of depressive symptoms. The BDI mean score was $13.15 \pm 10.01$. Thirty-seven lifetime and 36 current psychiatric disorders were diagnosed. After obtaining the patients' written informed consent, the genotype for the wolframin His611Arg polymorphism was determined.

Results R/R genotype carriers (10 patients) showed significantly higher drug consumption $(62.3 \pm 35.35$ vs. $40.64 \pm 25.00$ doses/month; $\mathrm{t}=2.266, p=0.028)$ and more severe depressive symptoms on the BDI questionnaire $(19.40 \pm 10.57$ vs. $11.66 \pm 9.40 ; \mathrm{t}=2.285, p=0.026)$ than non-R/R individuals (42 patients). No significant correlation was found between monthly drug consumption and depression score in our MOH patients $(r=0.137, p=0.334)$, even when their genotype was considered (R/R $r=0.172, p=0.635$; non-R/R $r=0.004, p=0.979)$.

Discussion Even if it has been reported that depression predicts disability and increased drug consumption in headache patients, in our $\mathrm{MOH}$ patients we found no direct correlation between depression scores and monthly intake of analgesic drugs. We believe that the increased drug consumption observed in $\mathrm{R} / \mathrm{R}$ carriers is a consequence of increased impulsivity and NS traits, irrespective of depression. Conclusions WFS1 R/R genotype appears to be an aggravating factor in drug overuse in $\mathrm{MOH}$ patients.

\section{HEADACHES: CLINICAL ASPECTS}

\section{ANALYSIS OF PATIENTS ATTENDING A SPECIALIZED HEADACHE CENTRE OF THE CALABRIA REGION OVER A ONE-YEAR PERIOD}

\section{${ }^{I}$ R. Iannacchero, ${ }^{2}$ V. Rispoli, ${ }^{1}$ E. De Caro}

${ }^{I}$ Regional Headache Centre, "Pugliese-Ciaccio" Hospital, Catanzaro, Italy; 'Department of Pharmacobiological Science, University "Magna Gracia" of Catanzaro; Catanzaro, Italy; e-mail: rosarioiann@tiscali.it

Objective Our aim was to analyse the diagnosis, prescribed medications and examinations conducted on patients attending a specialized Headache Centre of the Calabria Region over a one-year period.

Methods An audit of clinical records of patients attending the Headache Centre of the "Pugliese-Ciaccio" Hospital in Catanzaro, from January 2005 to January 2006, was conducted. Data regarding diagnosis, current medications, prescribed medications, and examinations conducted were collected.

Results Four hundred and fifteen patients attended the clinic during the audit period. Two hundred $(48 \%)$ of them were diagnosed with chronic daily headache (CDH), $160(39 \%)$ with migraine, $32(8 \%)$ with cluster headache, and $19(5 \%)$ with neuralgia headache TACs. All other diagnoses involved only one patient $(<1 \%)$ in each case. Most common medications used by patients with $\mathrm{CDH}$ at the time of initial consulta- 
tion were: analgesics (64\%), prophylactic drugs (17\%), triptans (14\%), and diazepam $(3 \%)$. Following the consultation, prophylaxis was prescribed for $51 \%$ of patients while triptans for only $1 \%$. At the time of initial consultation, patients with migraine were taking: analgesics $(59 \%)$, triptans $(26 \%)$, prophylactic drugs $(19 \%)$, and ergotamine (2\%). Following the consultation, prophylactic drugs was recommended for $75 \%$ of patients, triptans for $49 \%$, manipulation for $13 \%$, and analgesics for only $10 \%$. Examinations were arranged for 19 patients $(9 \%)$ and were negative in all cases.

Conclusions Most patients attending our headache centre were suffering from $\mathrm{CDH}$ or migraine. Cluster headache was rarely diagnosed. Despite this being a secondary care clinic, most patients were taking analgesics and relatively few specific acute therapies or prophylactic drugs. In contrast, triptan consumption markedly increased following consultation. In all clinical cases, further examinations were negative and were primarily conducted to reassure the patients.

\section{A VALIDATION STUDY OF AN ITALIAN VERSION OF THE "ID MIGRAINE"}

${ }^{I}$ F. Brighina, ${ }^{1} G$. Salemi, ${ }^{1} B$. Fierro, ${ }^{2} A$. Gasparro, ${ }^{3} A$. Balletta, ${ }^{3}$ A. Aloisio, ${ }^{4}$ G.B. La Pegna, ${ }^{5}$ G. Randisi, ${ }^{6}$ V. Saporito, ${ }^{7} G$. Calagna, ${ }^{8}$ F. Lanaia, ${ }^{8}$ R. Morana

${ }^{I}$ Department of Clinical Neurosciences, University of Palermo, Palermo, Italy; ${ }^{2}$ Neurology Unit, Villa Sofia Hospital, Palermo, Italy; ${ }^{3}$ Neurology Unit, Civic Hospital, Palermo, Italy; ${ }^{4}$ Department of Neurosciences, Caltanissetta, Italy; ${ }^{5}$ Neurology Unit, S. Elia Hospital, Caltanissetta, Italy; ${ }^{6}$ Headache Centre, ASL 6, Bagheria, Palermo, Italy; ${ }^{7}$ Headache Centre, Hospital of Partinico, Partinico, Palermo, Italy; ${ }^{8}$ Department of Neurosciences, University of Catania, Catania, Italy; e-mail: fbrighina@unipa.it

Introduction Migraine is a highly prevalent and disabling disease that is substantially underdiagnosed in primary care. Recently, the ID Migraine, a self-administered questionnaire, consisting of only three items, was shown to be a valid and reliable screening instrument for migraine in primary care in the U.S.A. The aim of the present study was to validate an Italian version of the "ID Migraine" questionnaire. Methods A total of 222 consecutive headache patients referring to eight headache centres in Sicily (Italy) completed an Italian version of the ID Migraine. The responses to the questionnaire were compared with the diagnosis of headache made by a headache specialist who did not know the results of the questionnaire. Sensitivity, specificity, positive and negative predictive value and accuracy level for migraine were calculated. Results The statistical analysis on 222 patients examined showed a very good performance of the ID Migraine with high sensitivity: 0.95 (95\% CI, 0.91-0.98), specificity: 0.72 (95\% CI, 0.62-0.82) and positive predictive value: 0.88 (95\% CI, 0.82-0.93). ID migraine also showed a very good accuracy level: 0.87 (95\% CI, 0.83-0.92).

Conclusions This study demonstrated "ID Migraine" to be a valid tool for migraine screening also in Italian patients referring to headache centres. If confirmed in a primary care setting, these results would establish the "ID Migraine" as a valid screening instrument for migraine in the Italian population.

\section{PATIENT COMPLIANCE TO COMPILATION OF THE HEADACHE DIARY}

L. Savi, P. De Martino, E. Zampella, L. Pinessi

Headache Centre, Neurology II, Department of Neurosciences, University of Turin, San Giovanni Battista Hospital, Turin, Italy; e-mail:lsavi@molinette.piemonte.it

Introduction One of the most important tools in the approach to a headache patient is the headache diary, whose compilation is a fundamental step in the formulation of the definitive diagnosis and in the organization of a therapy, which must be appropriate to the individual patient. The Headache Centre of the University of Turin began many years ago to use such a diary which, though modified and improved in the course of time, is still fundamentally the same.

Objective The purpose of this study was to evaluate the patient compliance to the compilation of the headache diary.

Materials and methods A total of 575 patients (133 males and 442 females, age range 18-85 years) attending for the first time the Headache Centre of the University of Turin in the period January 1 December 31, 2004, were enrolled in the study. They all suffered from various kinds of primary headache diagnosed according to the International Headache Society criteria (ICHD-II).

The usual diagnostic approach to the patients at the Headache Centre of the University of Turin starts with a first visit, in the course of which as much information as possible is collected from the patient's interview, and a symptomatic therapy is suggested. This is followed after three months by a second visit, when a more definitive diagnosis is formulated and, if it is deemed necessary, prophylactic therapy is suggested to the patient.

The patients are given the headache diary at the first visit. They are instructed how to complete it and are asked to be as careful as possible in its compilation.

They have to record many different characteristics of their headache (pain, duration, intensity, localization, aggravation by routine physical activity, presence of other symptoms, and drugs used).

It was evaluated how the diary had been filled in by all the patients who came to the second visit, considering their age, level of instruction, type of work, and comprehension of the language.

Results After the first visit 393 patients came back, while 182 did not. Among those who came back, $324(82.45 \%)$ filled the diary completely, while the remaining $69(17.55 \%)$ filled it in only partially or did not fill it in at all.

Discussion and conclusions On the basis of these data it is possible to affirm that the majority of patients showed a very satisfactory compliance to the diary compilation.

\section{PATIENT COMPLIANCE TO THE DIAGNOSTIC APPROACH IN HEADACHE}

L. Savi, P. De Martino, E. Zampella, L. Pinessi

Headache Centre, Neurology II, Department of Neurosciences, University of Turin, San Giovanni Battista Hospital, Turin, Italy; e-mail:lsavi@molinette.piemonte.it

Introduction The usual diagnostic approach to the patients at the Headache Centre of the University of Turin starts with an initial visit, in the course of which as much information as possible is collected from the patient's interview, and a symptomatic therapy is suggested. This is followed after three months by a second visit, when a more definitive diagnosis is formulated and, if it is deemed necessary, prophylactic therapy is suggested to the patient.

According to some critics, this diagnostic approach is too long, and the patients do not like it.

Objective The purpose of this study was to evaluate how many patients do not come back for the second visit that forms the usual pattern of our approach, and why they do not return, to clarify if this criticism is correct.

Materials and methods A total of 575 patients (133 males and 442 females, age range 18-85 years), attending for the first time the Headache Centre of the University of Turin in the period January 1 December 31, 2004, were enrolled in the study. All patients suffered from various kinds of primary headache diagnosed according to the International Headache Society criteria ICHD-II. It was determined if they had returned for the second visit or not. All patients who did not return were contacted by phone to explain their reasons.

Results After the first visit $393(68.35 \%)$ patients returned, while 182 $(31.65 \%)$ did not. The principal reasons for their not returning, as reported by those same patients were: the therapy was effective at the first attempt or viceversa; headache attacks reduced their number after the 
first visit; the diary compilation was too complicated (it was a very difficult task for the patients with chronic headache); the diary was a nuisance because the headache attacks were so few; the fee paid for the visit was too high and the visit itself did not offer a solution to their problem; and the patient did not like the doctor's approach at the first visit. Discussion and conclusions On the basis of these data it is possible to note that the majority of the patients accept and understand this diagnostic approach, so the criticism towards it does not seem to be realistic. Considering the most frequent motivations reported by the patients who did not come back, it appears likely that providing more information could improve the situation.

\section{PREVALENCE OF ARTERIAL HYPERTENSION IN THE REL- ATIVES OF MIGRAINE PATIENTS}

${ }^{I}$ F. De Cesaris, ${ }^{1}$ U. Pietrini, ${ }^{1}$ B. Anselmi, ${ }^{2}$ A. Del Bene, ${ }^{1}$ E. Del Bene ${ }^{1}$ Headache Centre, Department of Internal Medicine and ${ }^{2}$ Department of Neurological and Psychiatric Sciences, University of Florence, Florence, Italy; e-mail:f.decesaris@dmi.unifi.it

Introduction Migraine and some cardiovascular disorders should have common pathogenic mechanisms, and genetic factors should play an important role in the possible development of migraine in a subject with a positive gender for cardiovascular disorders [1]. Moreover, in the clinical history of patients affected by migraine the presence of arterial hypertension in their relatives is frequently observed.

Objective The aim of our study was to assess how common this phenomenon is in a population of children and adults.

Materials and methods The study sample included 190 patients affected by episodic migraine without aura (according to ICHD-II criteria, 2004 [2]), without other forms of headache and without other comorbidity. Seventy-four subjects $(38.94 \%, 50 \mathrm{~F}, 24 \mathrm{M}$, age range 10-72 years, mean age 39.05 years - Group A) did not refer gender positivity for arterial hypertension, while $116(61.06 \%, 86 \mathrm{~F}, 30 \mathrm{M}$, range 6-77 years, mean age 33.97 years - Group B) referred the presence of arterial hypertension in almost one relative of first (parents), second (sister, brother, grandparents) or third degree (uncles or aunts). Before the visit, a dedicated, specific and simple questionnaire was given to the patient for compilation.

Results In Group B, 83 subjects referred the presence of arterial hypertension in one or both first degree relatives, 76 in second (with or without first) degree relatives, 20 in third (with or without second and first) degree relatives. The mean number of attacks per month was 4.51 in Group A and 5.49 in Group B.

Conclusions This study has two limitations, the small patient cohort and the absence of a control group (without migraine). Nonetheless, we can suppose that migraineurs have a remarkable prevalence of relatives with arterial hypertension, according to previous studies, and that the number of attacks seems to be higher in the group with gender positivity for arterial hypertension.

References

1. Lanzi G, Termine C, Rossi M et al (2003) Are vascular disorders more prevalent in the relatives of children and adolescents with migraine? Cephalalgia 23:887-891

2. Headache Classification Subcommittee of the International Headache Society (2004) The International Classification of Headache Disorders, 2nd edn. Cephalalgia 24[Suppl 1]:9-160

\section{FOOD INTOLERANCE IN MIGRAINE}

${ }^{I}$ V. Pizza, ${ }^{2}$ M. Mainenti, ${ }^{1}$ G. Volpe, ${ }^{1}$ E. Lamaida, ${ }^{I}$ S. Iannuzzi, ${ }^{l}$ A. Agresta, ${ }^{3}$ A. Capasso, ${ }^{1,3}$ A. Bisogno, ${ }^{3}$ A. Bianchi, ${ }^{4}$ C. Colucci d'Amato ${ }^{I}$ Neurophysiopathology Service, Headache Centre, S. Luca Hospital and ${ }^{2}$ Endocrinology Service, ASL SA/3, Vallo della Lucania, Salerno, Italy; ${ }^{3}$ Pharmacology Department, University of Salerno, Salerno, Italy; ${ }^{4}$ Neurosciences Department, Second University of Naples, Naples, Italy; e-mail:vpizza@libero.it
Objective Several factors can trigger migraine; among them, dietary factors play a very important role in the onset of migraine attacks. The aim of our study was to evaluate the incidence of food intolerances in a group of migraineurs, by using the Cytotoxic test.

Materials and methods Thirty consecutive patients suffering from migraine presenting to the Headache Centre of S. Luca Hospital, Vallo della Lucania (SA) were examined. Twenty-three were women, mean age was 28.2 years, range $13-47$ years; 7 were men, mean age was 39.33 years, range $28-62$ years. The Cytotoxic test is capable of identifying the presence of specific food intolerances by observing the appearance, the size, the shape or the integrity of leukocytes exposed to extracted food antigens or other materials derived from specific foods. Results An intolerance was found to: tyramine in 12 women $(52.17 \%)$ and 3 men (42.85\%); milk, 3 women (13.05\%); yeast, 4 women $(17.39 \%)$ and 1 man $(14.28 \%)$; solanaceae, 4 women $(17.39 \%)$ and 1 man (14.28\%); coffee, 5 women $(21.74 \%)$; cocoa, 5 women $(21.74 \%)$; tea, 2 women (8.69\%); eggs, 1 woman $(4.35 \%)$ and 1 man $(14.28 \%)$; pork, 1 woman $(4.35 \%)$; and sugar, 1 woman $(4.35 \%)$.

Conclusions Our study showed a high incidence of food intolerance in migraineurs (in women more than in men). The dietary factors which gave more significant results were tyramine, yeast, solanaceae, coffee and cocoa. These results are in agreement with those of other studies found in the literature, proposing tyramine, coffee and cocoa as very important migraine-precipitating factors. Conversely, there is little evidence of a comorbidity between migraine and intolerance to solanaceae. For this reason, further studies are warranted to confirm this hypothesis.

\section{ALTERATIONS IN GLUCOSE-INSULIN METABOLISM IN MIGRAINEURS: PROSPECTIVE STUDY ON THE CLINICAL IMPACT OF DIET ON MIGRAINE SEVERITY AND META- BOLIC DATA IN A MIGRAINEUR POPULATION - PRELIMI- NARY RESULTS}

${ }^{1,2}$ C. Cavestro, ${ }^{3}$ A. Rosatello, ${ }^{4}$ G.M. Micca, ${ }^{1}$ G. Asteggiano

${ }^{1}$ Department of Neurology and ${ }^{2}$ Headache Centre, "San Lazzaro" Hospital, A.S.L. 18, Alba, Cuneo, Italy; ${ }^{3}$ Department of Endocrinology, "San Lazzaro" Hospital, Alba, Italy; ${ }^{4}$ Laboratory of Biochemistry, "San Lazzaro" Hospital, Alba, Cuneo, Italy; e-mail: cicaves@ tin.it

Introducion Migraine is characterized by a complex biochemical dysfunction attributed to a disorder of the trigeminal and hypothalamic pathways. Impairment of glucose and insulin metabolism has been reported in migraine [1], and insulin alterations seem to be specific to migraineurs. Objective The main aim was to verify if a proper diet, eventually associated with a insulin-sensitizing drug where indicated, could modify the headache severity together with the glucose-insulin metabolic alteration in migraineurs who presented alterations in glucose-insulin metabolism.

Patients and methods The study population included all migraineurs consecutively seen at the headache centre of our hospital during a oneyear period; all the recruited people underwent a general blood test and a standard oral glucose tolerance test (OGTT) after a 12-hour fasting period, measuring levels of glucose and insulin. A daily fragmented low-percentage carbohydrate diet was suggested to those patients who showed an alteration in glucose and or insulin metabolism. Each patient was followed-up during a 3-6 month period, and another standard OGTT was performed, following a 3-6 month period of diet after a 12-hour fasting period, measuring levels of glucose and insulin. Evaluation of headache severity was expressed in severity index, calculated on the basis of number of days with headache and each day headache severity in 1-3 severity scale (1 mild, 2 moderate, 3 severe). Results In a 12-month period, we recruited 173 migraineurs (138 women, 35 men), 132 patients had a metabolic glucose-insulin alteration (102 women, 30 men), and 41 patients had normal metabolic profile. Until now, 64 patients have been followed-up for at least 3-6 months. Headache severity index of the studied population was 31 
$(\mathrm{SD} \pm 27)$ before diet and 12 after diet $(\mathrm{SD} \pm 21)(p<0.000)$. Fasting blood glucose levels (FBG) and after OGTT (in $\mathrm{mg} / \mathrm{dL}$ ) were slighty lower after dieting (before diet, FBG: 96, at 30 minutes: 143, at 60 minutes: 113, at 120 minutes: 93 ; after dieting, FBG: 99 , at 30 minutes: 131, at 60 minutes: 104 , at 120 minutes: $83 ; p<0.3$ ). Migraineurs after dieting presented significantly lower insulin levels at any time of the test, both fasting and after glucose loading (before dieting, FBG: 10, at $30 \mathrm{~min}-$ utes: 100, at 60 minutes: 99, at 120 minutes: 55; after dieting, FBG: 7, at 30 minutes: 74, at 60 minutes: 75, at 120 minutes: 38; $p<0.1$ ).

Discussion and conclusions A daily fragmented low percentage carbohydrate diet can be useful as migraine non-pharmacological therapy. A clinically positive impact of diet correlates with glucose and even more with insulin levels, both fasting and after glucose loading. High insulin levels are recorded in migraineurs, and these data seem to be specific to this headache type. These findings are in keeping with recent reports on the effects of insulin on brain functions and lend support to the possible involvement of insulin in the pathogenesis of migraine [2].

References

1. Rainero I, Limone P, Ferrero M et al (2005) Insulin sensitivity is impaired in patients with migraine. Cephalalgia 25:593-597

2. Porte D, Baskin DG, Schwartz MW (2005) Insulin signaling in the central nervous system: a critical role in metabolic homeostasis and disease from C. elegans to humans. Diabetes 54:1264-1276

\section{POST-PRANDIAL MOTILITY INDEX IS SIGNIFICANTLY REDUCED IN MIGRAINE PATIENTS WITH DYSPEPTIC SYMPTOMS}

${ }^{l}$ E. Pucci, ${ }^{2}$ M. Di Stefano, ${ }^{1,3}$ M. Bartolo, ${ }^{1} S$. Buscone, ${ }^{2}$ E. Miceli, ${ }^{2}$ G.R. Corazza, ${ }^{1}$ G. Sandrini, ${ }^{1,3}$ G. Nappi

${ }^{1}$ University Centre for Adaptive Disorders and Headache (UCADH), University of Pavia, IRCCS “C. Mondino" Foundation, Pavia, Italy; ${ }^{2}$ I Department of Medicine, University of Pavia, IRCCS "S. Matteo" Hospital, Pavia, Italy; ${ }^{3}$ Department of Neurology and ORL, University of Rome "La Sapienza”, Rome, Italy; e-mail: ennio.pucci@mondino.it

Background and aims We have recently shown that in patients with migraine and dyspepsia the intake of a low-caloric low-fat meal induces a significant reduction of post-prandial discomfort thresholds. This abnormality is not evident in patients with functional dyspepsia, thus suggesting that post-prandial reduction of discomfort thresholds may characterize migraine patients. As variations of gastric motility index (MI) were described in a subset of dyspeptic patients, the aim of this study was to evaluate whether abnormalities of this parameter may have a role in the pathophysiology of dyspeptic symptoms in migraine patients.

Materials and methods Eighteen patients with migraine without aura, diagnosed according to ICHD-II criteria and dyspeptic symptoms (11 $\mathrm{F}$, mean age $32 \pm 5$ years), 16 patients with functional dyspepsia, diagnosed according to Rome II criteria ( $10 \mathrm{~F}$, mean age $39 \pm 5$ years) and 16 healthy volunteers (HV) $(9 \mathrm{~F}$, mean age $28 \pm 6$ years) underwent gastric barostat test, which monitors gastric motor activity (contraction or relaxation) as changes in intraballoon volume (reduction or increase, respectively) at a constant intrabag pressure. After an overnight fast, a double lumen polyvinyl tube with an adherent, infinitely compliant plastic bag (1200 mL capacity), finely folded, was inserted into the stomach through the mouth. The polyvinyl tube was connected to a computer-driven programmable volume-displacement barostat device. During the test we evaluated gastric accommodation reflex and phasic contractility, quantified using MI during fasting and during the first and second 30-min period after administration of a low-caloric liquid meal. A baseline reconstruction was performed using a computerised algorithm and MI was calculated as the area between the signal and the baseline normalised over time.

Results Both in dyspeptic patients with migraine and without migraine, the mean increase of post-prandial gastric volume was indicative of a physiological accommodation. Fasting MI was similar in patients (migraine $27.5 \pm 7.1 \mathrm{~mL}$ x min; dyspepsia $26.9 \pm 4.6 \mathrm{~mL} \mathrm{x} \mathrm{min)} \mathrm{and} \mathrm{HV}$ $(24.8 \pm 4.3 \mathrm{~mL} \mathrm{x} \mathrm{min})$. On the contrary, during the first post-prandial $30-$ min period, post-prandial MI was significantly reduced $(p<0.003$, ANOVA) in patients with migraine $(13.4 \pm 4.8 \mathrm{~mL} \times \mathrm{min})$ than in $\mathrm{HV}$ $(19.6 \pm 6.6 \mathrm{~mL} \times \mathrm{min})$ and in dyspeptic patients $(22.6 \pm 7.4 \mathrm{~mL} \mathrm{x} \mathrm{min})$. Conclusions Our results show that patients with migraine and dyspeptic symptoms suffer from an alteration of post-prandial gastric sensorimotor function. In addition to the previously shown post-prandial hypersensitivity, a reduction of post-prandial MI is also present.

\section{EPIGONE MIGRAINE VERTIGO (EMV): A LATE MIGRAINE EQUIVALENT}

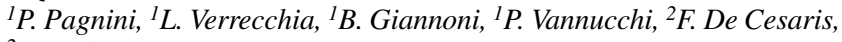
${ }^{2}$ E. Del Bene

${ }^{I}$ Audiology Unit, Department of Surgical Oto-neuro-ophthalmological Sciences, ${ }^{2}$ Headache Centre, Department of Internal Medicine, University of Florence, Florence, Italy; e-mail: audiologia@dfc.unifi.it

Introduction The term Migrainous Vertigo (MV) [1] describes all the vertiginous and postural disturbances that are variably expressed by migrainous subjects. In 1988, Pagnini [2] proposed a classification of the vestibular symptoms in migraine using a specific criterion: the temporal relationship between headache and vertigo. According to this classification we can find two large groups of MV: the associated and the equivalent. The first represents the vestibular disorder that adds to headache. The second represents the vestibular crisis that substitutes the single spell of headache. The equivalent form can occur before the onset of headaches (precocious equivalent). It could appear during the headache period (intercritical equivalent), or it can develop after the headache ceases (epigone equivalent). This study concerns the latter form.

Subjects and methods In a clinical observation of 13 years at the Audiological Clinic of the University of Florence, we studied 28 migrainous patients with some features of recurrent vestibular disorders: they suffered from late vertigo with onset only after headache had ceased; their vertigo was not specific for other recurrent otoneuropathies; there were no specific radiological signs or blood risk factors. With a follow-up at least of two years, we studied this patient group with audiovestibular and neurological examinations and imaging, focusing on the characteristics of headache and vertigo and how they interchanged between each other.

Results and discussion Females represented $82 \%$ of the study group, mean age of headache onset was 18 years and for vertigo was 38 years. Migraine without aura was present in $78 \%$ of patients, kinetosis in $78 \%$. The vertigo described by the patients was variable: $64 \%$ of patients referred exclusively rotatory crises (mean duration 15 hours); $14 \%$ described dizziness or postural instability (mean duration 73 hours); $22 \%$ suffered from a combination of these features. The headache passed to vertigo with a free interval in $26 \%$ of patients. A period of alternation of headache and vertigo was present in $29 \%$ of patients. In $45 \%$ of patients headache passed directly into vertigo. There were no specific signs from audiovestibular examination during intercritical periods, but in $36 \%$ we found a vestibular hyperreflectivity during stimulation. We saw a specific highly repetitive behaviour for this new entity: the response to migraine prophylaxis. Vertigo improved in all patients, furthermore, full recovery from vestibular symptoms was obtained in $68 \%$ of patients.

Conclusions With this clinical observational study, we attempted to characterize a new clinical picture of migraine, the epigone migrainous vertigo, which should be considered when considering vertigo in migraine.

References

1. Kayan A, Hood JD (1984) Neuro-otological manifestations of migraine. Brain 107:1123-1142

2. Pagnini P, Verrecchia L, Giannoni B, Vannucchi P (2003) [Hemicrania-related vertigo (HV)]. Acta Otorhinolaryngo Ital 23[5 Suppl 75]:19-27 (Article in Italian) 


\section{MUSCLE TENDERNESS IN CRANIOMANDIBULAR AND CERVICAL REGIONS AND PSYCHOMETRIC CORRELATES IN A POPULATION OF MIGRAINE PATIENTS WITH AND WITHOUT CHRONIC TENSION-TYPE HEADACHE AND HEALTHY CONTROLS \\ ${ }^{1} V$. D'Agostino, ${ }^{2} G$. Bruti, ${ }^{3} A$. Rivaroli, ${ }^{2} G$. Fiocco, ${ }^{1} F$. Fattapposta,} ${ }^{3} P$. Cascone, ${ }^{3} G$. Iannetti, ${ }^{2} R$. Cerbo

${ }^{1}$ Department of Neurology and Ear, Nose and Throat Specialist, ${ }^{2}$ Headache Section, ${ }^{3}$ Oromaxillo-facial Pain Section of the Pain Centre "Enzo Borzomati", Policlinico Umberto I, University of Rome "La Sapienza”, Rome, Italy; e-mail: gianluca.bruti@uniromal.it

Background A relationship has been shown between pericranial muscle tenderness and psychiatric disorders in migraine patients. In particular, this association has been observed in patients with both migraine and chronic tension-type headache (CTTH).

Objective The aim of the study was: 1) to evaluate the function of the temporomandibular system and of the cervical spine in a population of migraineurs with and without CTTH; 2) to evaluate the relationship between muscle tenderness, personality profile and psychiatric symptoms in migraine patients with and without CTTH.

Materials and methods Migraineurs with and without CTTH were consecutively recruited according to IHS criteria (ICHD-II). A healthy control group was also recruited. All subjects of the study were submitted to: 1) clinical investigation of the temporomandibular system according to the "Craniomandibular Index" (CMI); 2) pericranial muscle evaluation according to "Total Tenderness Score" (TTS); 3) physical examination of the cervical spine (PECS); and 4) electrognathography (EGG) of the temporomandibular joint. As regards personality profile and psychiatric symptoms, the following tests were administered: Tridimensional Personality Questionnaire (TPQ), Toronto Alexithymia Scale - 20 items (TAS-20), State and Trait Anxiety Inventory (STAI-12 ), and Beck Depression Inventory (BDI). All migraineurs underwent Migraine Disability Assessment (MIDAS) and Headache Impact Test 6 items (HIT-6) for evaluation of migraine disability.

Results Forty-two migraineurs (mean age $35 \pm 10$ years), 35 migraineurs with CTTH (mean age $34 \pm 12$ years) and 32 healthy controls (mean age $32 \pm 11$ years) were admitted to the study. All migraineurs showed significantly higher scores in CMI, TTS and PECS than healthy controls $(p<0.05)$. Comparing EGG data between migraineurs and healthy controls, we observed in the migraine population a reduction of mandibular movement associated with muscle rigidity $(p<0.05)$. In regard to the psychometric data, migraineurs showed higher scores in BDI and STAI, Harm Avoidance dimension of the TPQ and TAS-20 than healthy controls $(p<0.05)$. All clinical and psychometric findings cited above were more evident in migraineurs with CTTH than those without $(p<0.05)$ Duration of illness, frequency of attacks as well as MIDAS and HIT-6 scores were positively correlated with the total number of craniomandibular and cervical tender points $(p<0.05)$.

Conclusions Migraine patients present subclinical temporomandibular and cervical spine disorders. These clinical findings are more evident in migraineurs with CTTH and are associated with psychiatric symptoms and with a peculiar personality profile. It might be important to identify these clinical features not only for the therapeutic implications but also to prevent and understand the pathophysiological mechanisms that may lead to chronic migraine.

\section{MIGRAINE AS PRESENTING SYMPTOM OF CEREBRAL AUTOSOMAL DOMINANT ARTERIOPATHY WITH SUBCOR- TICAL INFARCTS AND LEUKOENCEPHALOPATHY (CADASIL)}

${ }^{I}$ C. Sarti, ${ }^{1}$ F. Pescini, ${ }^{1}$ V. Palumbo, ${ }^{1} A$. Poggesi, ${ }^{2} S$. Bianchi, ${ }^{2}$ M.T. Dotti, ${ }^{2}$ A. Federico, ${ }^{1}$ D. Inzitari, ${ }^{1}$ L. Pantoni

${ }^{1}$ Department of Neurological and Psychiatric Sciences, University of Florence, Florence, Italy; ${ }^{2}$ Department of Neurological and Behavioural Sciences, University of Siena, Siena, Italy; e-mail: pantoni@unifi.it
Background CADASIL is an inherited (autosomal dominant) vascular disease clinically characterized by recurrent transient ischemic attacks and/or strokes, migraine, mood disorders, cognitive impairment, and seizures. Radiological hallmarks are diffuse white matter abnormalities and/or lacunar infarcts visible on MRI or CT. Genetic mutations are located on chromosome 19p13.1 on the Notch3 gene.

Migraine is the symptom of onset of the disease in $40 \%$ of cases [1] and has been described in up to $77 \%$ of patients in the advanced phase of the disease. Migraine with aura has been reported in up to $40 \%$ of cases. About $30 \%$ of patients have atypical aura, and atypical plus typical aura has been described in $27 \%$ of cases [2].

Methods We describe the clinical characteristics of 14 consecutive CADASIL patients ( 5 males) belonging to 9 unrelated families.

Results The time of the first neurological evaluation was at an advanced age (mean $51 \pm 15$ years). Onset of the disease was retrospectively judged to be earlier (mean age 29 years \pm 16 ) with migraine as the presentation symptom in $64 \%$ of patients. On presentation no patient had migraine with aura. Migraine was present in $86 \%$ of patients in the advanced phase of the disease. Three patients (21\%) had migraine with aura, 2 of them (14\%) had typical and prolonged aura. The other clinical features were TIA/strokes (57\%), cognitive deficits $(71 \%)$, and mood disturbances $(79 \%)$.

All patients presented confluent or partly-confluent MRI white matter changes with the exception of 2 young patients ( 26 and 28 years) clinically characterized by isolated migraine. Involvement of the temporal lobes was variable in terms of severity. Family history was positive for at least one CADASIL clinical hallmark in all cases. Notch3 mutations involved exon 11 (3 families), 19 (2 families), and exons 3, 4, 6, and 10 in one family each.

Conclusions CADASIL appears to be an underestimated disease. The phenotypic spectrum is variable ranging from pauci-symptomatic cases to severe disease. Migraine is frequently the earliest symptom of the disease occurring at a relatively young age. CADASIL should be suspected in patients with migraine who have MRI white matter alterations of moderate to severe degree and a positive family history for at least one clinical hallmark of the disease.

References

1. Desmond DW, Moroney JT, Lynch T et al (1999) The natural history of CADASIL: a pooled analysis of previously published cases. Stroke 30:1230-1233

2. Vahedi K, Chabriat H, Levy C et al (2004) Migraine with aura and brain magnetic resonance imaging abnormalities in patients with CADASIL. Arch Neurol 61:1237-1240

\section{PRIMARY CHRONIC HEADACHES IN ITALY: ANALYSIS OF DIAGNOSES WITH THE AID OF A DEDICATED SOFTWARE BASED ON ICHD-II CRITERIA}

A. Baldi, F. Coppola, I. Corbelli, M.L. Mancini, C. Rossi, P. Calabresi, P. Sarchielli

Headache Centre, Neurologic Clinic, Department of Medical Surgical Specialties and Public Health, University of Perugia, Perugia, Italy; $e$ mail:baldianto@libero.it

Background We recently developed a software, the Primary Headaches Analyser 1.0 INT (PHA), to analyse the clinical data from patients affected by primary headaches [1]. This programme is based exclusively on the International Classification of Headache Disorders 2nd edition (ICHD-II) criteria [2]. This software examines all the diagnoses of primary headaches on the basis of the variables needed to fulfil these mandatory criteria.

Methods We tested the diagnoses of chronic primary headaches with the aid of the software PHA, by entering and analyzing data reported in the clinical chart and headache diaries from 200 consecutive patients affected by primary chronic headaches and evaluating the corresponding output diagnoses. 
Results The diagnosis of 1.5.1 Chronic migraine was obtained in 68 cases $(34 \%)$, and that of probable 1.6.5 Chronic migraine +8.2 .8$ Probable medication-overuse headache in 46 (23\%). 2.3 Chronic tension-type headache and 2.4.3 Probable chronic tension-type headache +8.2 .8 Probable medication-overuse headache were diagnosed in 24 $(12 \%)$, and $2(1 \%)$ patients, respectively. Probable medication-overuse headache $(n=46)$ included combination analgesics $(n=12)$, analgesics $(n=11)$, triptans $(n=9)$, a combination of acute medications $(n=7)$, opioids $(n=4)$, and ergot derivatives $(n=3)$. In the two patients with a diagnosis of 2.4.3 Probable chronic tension-type headache + 8.2.8 Probable medication-overuse headache, combination analgesics were responsible for medication overuse. Moreover, 4 and 12 patients, respectively, received both the diagnosis of 1.5.1 Chronic migraine +2.3 Chronic tension-type headache and of 1.6.1 Probable migraine without aura +2.3 Chronic tension-type headache. In the remaining 44 cases $(22 \%)$, none of the chronic primary headaches defined by ICHD-II were given as an output diagnosis by our software. This was mainly due to the fact that insufficient criteria were fulfilled for the diagnoses of 1.1 Migraine without aura + 1.5.1 Chronic migraine or, more infrequently, 2.3 Chronic tension-type headache.

Output diagnoses included 1.1 Migraine without aura, 1.6.1 Probable migraine without aura, 2.4.1 Probable infrequent episodic tension-type headache + 2.4.2 Probable frequent episodic tension-type headache, and 14.1 Headache not elsewhere classified. None of the patients whose clinical sheets were examined received a diagnosis of 4.7 Hemicrania continua or 4.8 New daily-persistent headache.

Conclusions Our software (PHA) allowed the diagnoses of chronic migraine or chronic tension-type headache or their probable forms in the case of medication overuse in $78 \%$ of the patients with more than 15 days per month examined. In the remaining cases, the inability to provide the above diagnoses can be explained in about one quarter of cases by the fact that the mandatory criteria for both diagnoses are too stringent and do not reflect modifications of the headache patterns in these chronic forms.

References

1. Sarchielli P, Pedini M, Alberti A et al (2005) Application of ICHD 2nd edition criteria for primary headaches with the aid of a computerised, structured medical record for the specialist. J Headache Pain 6:205-210

2. Headache Classification Subcommittee of the International Headache Society (2004) The International Classification of Headache Disorders, 2nd edn. Cephalalgia 24[Suppl 1]:9-160

\section{DISABILITY AND CHRONIC DAILY HEADACHES: WHO-DAS II VERSUS OTHER SPECIFIC AND NON SPECIFIC DIS- ABILITY TOOLS}

${ }^{1}$ M.G. Buzzi, ${ }^{1}$ D. Cologno, ${ }^{3}$ D. Mei, ${ }^{3}$ G. Di Trapani, ${ }^{1,2}$ C. Caltagirone, ${ }^{1,2}$ U. Nocentini

${ }^{1}$ Headache Centre, IRCCS Santa Lucia Foundation, Rome, Italy; ${ }^{2}$ Institute of Neurology, Neuroscience Department, University of Rome "Tor Vergata”, Rome, Italy; ${ }^{3}$ Headache Centre, Institute of Neurology, Catholic University, Rome, Italy; e-mail: mg.buzzi@hsantalucia.it

Introduction The present study was conducted in order to compare the sensitivity of the newly developed World Health Organization Disability Assessment Schedule II for detecting disability due to chronic pain with two established instruments, one generic (SF-36) [1] and one specific for headache (HIT-6) in patients affected by chronic daily headache $(\mathrm{CDH})$ with and without analgesics overuse. WHO-DAS II consists of 36 items, grouped into 6 domains, 5 being related to personal activities and 1 to social life events: D1 communication abilities; D2 mobility impairment; D3 self care; D4 interpersonal relationship; D5 familial/working daily activities; D6 social life participation rate. Methods Forty consecutive patients (30 females and 10 males), affected by $\mathrm{CDH}$ with or without analgesic overuse and observed during a 6-month period were interviewed through SF-36, HIT-6 and the 36-item version of WHO-DAS II scales, each one in its validated
Italian version. Headache diagnosis was made according to ICHD-II criteria [2]. No patient had taken prophylactic medications for headache during the 6 months preceding the observation. Statistical analysis included partial correlation tests between items of the three instruments and alfa Cronbach parameter for evaluation of WHODAS II internal consistency.

Results A positive correlation was found between all items of SF-36 and some items of WHO-DAS II, in D4, D5 and D6 domains, with statistically significant differences $(p<0.01)$. D5 and D6 WHO-DAS II domains correlated positively with all HIT-6 items $(p<0.01)$, D4 domain correlated with items $3,4,5,6$ of HIT-6 $(p<0.01)$, D1 domain with items $4,5,6(p<0.05)$ and D2 domain with item 3 only $(p<0.05)$. Discussion and conclusions The above findings show that WHO-DAS II is comparable to SF-36 and HIT-6 as a tool for disability measurement. No correlations were found between WHO-DAS II items and headache clinical features, such as disease duration or duration of analgesic overuse. Some SF-36 items, instead, showed a statistically significant correlation with duration of illness, but not with the duration of analgesic overuse. To identify internal consistency between all items of WHO-DAS II, we used alfa Cronbach parameter. Its value was between 0.8 and 0.9 for all domains analysed indicating a high internal consistency, which supports the use of WHO-DAS II as a sensitive instrument for detecting disability in headache patients, In particular, it may prove to be a valuable measurement tool for disability in chronic headache patients, and it could be useful for indicating effectiveness of therapy and guiding clinical management in these patients.

References

1. Apolone G, Mosconi P (1998) The Italian SF-36 Health Survey: translation, validation and norming. J Clin Epidemiol 51:1025-1036

2. Headache Classification Subcommittee of the International Headache Society (2004) The International Classification of Headache Disorders, 2nd edn. Cephalalgia 24[Suppl 1]:9-160

\section{CLINICAL CHARACTERISTICS OF MEDICATION-OVERUSE HEADACHE}

G. Relja, A. Granato, M. Zorzon, Z. Bisin, G. Nider, G. Pizzolato

Department of Clinical Medicine and Neurology, Headache Centre, University of Trieste, Trieste, Italy; e-mail: antonio_granato@hotmail.com

Introduction The International Classification of Headache Disorders (ICDH-II, 2004) remarkably modified the diagnostic criteria of medication-overuse headache ( $\mathrm{MOH})$, and also proposed clinical criteria to differentiate headache between different overused medications. Subsequently, these clinical criteria were excluded in the revision of the International Headache Seminar held in Copenhagen in March 2004, due to lack of reliable data from the literature.

Objective The aim of the present study was to analyse, for the first time in a systematic way, the clinical characteristics of headache in a sample of patients suffering from $\mathrm{MOH}$.

Patients and methods We studied 51 patients with chronic daily headache who took analgesics $\geq$ once per day for more than three months, and were treated for detoxification as in-patients. The study included 46 women $(90.2 \%)$ and 5 men $(9.8 \%)$, with a mean age of 53 [SD 12] years. The patients were divided according to the medication overused. The quality, the site, the severity, and the accompanying phenomena were evaluated, taking into account also the characteristics of the pre-existing headache and the response to the medication used. The data were analysed using the Statistical Package for the Social Sciences (SPSS 12.0).

Results The overused medications were: combination analgesics in 20 patients $(39.2 \%)$, simple analgesics in 17 patients $(33.3 \%)$, triptans in 10 patients $(19.6 \%)$, and ergotamine in 4 patients $(7.8 \%)$. The more common pre-existing headache was migraine without aura (39 patients, $76.5 \%)$. The pain was more frequently pulsating (28 patients, $54.9 \%)$, bilateral (29 patients, 56.9\%), and of severe intensity (34 patients, $66.7 \%$ ). Accompanying phenomena were present in 43 patients 
(84.3\%), the more frequent being nausea associated with phonophobia and photophobia. The quality, site and intensity of pain, and the type of accompanying phenomena did not differ significantly among overusers of different medications $(p=\mathrm{NS})$. The overused medications were effective in reducing headache and the mean time to the resolution of pain was $72[\mathrm{SD}=87]$ minutes.

Conclusions The headache in this sample of patients with $\mathrm{MOH}$ was more frequently pulsating, bilateral, severe and associated with nausea and phonophobia and photophobia. The clinical characteristics were not different according to the various types of overused medications, supporting the revision of the International Headache Seminar of Copenhagen (2004). The pre-existing headache and the response to overused medications did not influence the characteristics of MOH.

\section{SUICIDE RISK IN CHRONIC DAILY HEADACHE}

S. de Filippis, E. Salvatori, E. Nittoli, G. Coloprisco, I. Farinelli, P. Martelletti

University of Rome "La Sapienza", II School of Medicine, Regional Referral Headache Centre, Sant'Andrea Hospital, Rome, Italy; e-mail: sergio.defilippis@uniroma1.it

In the Western world, $2 \%-4 \%$ of deaths are due to suicide, incidents and murders. Suicide is experienced as a predictable, and therefore preventable death; its occurrence causes a sense of anguish in relatives and frustration and impotence in physicians. Chronic somatic pathologies, such as diabetes, cardiopathy, cancer, stroke and correlated problems like pain, weight loss, insomnia etc. increase vulnerability and predispose to the risk of suicide. Our aim in this study was to evaluate the presence of both humoral disorders and suicide risk in 300 patients affected by chronic daily headache and medication-overuse headache, who were observed for 24 months in our Centre.

We based our work relying upon interviews, collection of anamnestic data related to the premorbid period, information given by patients concerning the sufferers' behaviour during the day, attitudes towards others, maintaining of previous interests, and modifications of the biological rhythm.

Moreover, psychometric tests were administered, revealing a remarkable correlation between humoral disorders and headache, impairment of working activity, relationships and family life.

Through structured interviews with patients and family, the presence of both depressive symptomatology and suicide ideation before the appearance of chronic daily headache has been evaluated.

Finally, our purpose was also to establish if, unlike other pathologies, suicide risk was high or remained just intentional.

Preliminary data indicate 42 patients $(21 \%)$ with positive results; more specific tests (SSI, BRFL) were performed in order to quantify the importance, intensity and pervasiveness of suicide ideation.

\section{CAse Reports}

\section{DIFFICULTIES IN MIGRALEPSY DIAGNOSIS: A CASE REPORT}

${ }^{I}$ G. Sances, ${ }^{1} N$. Ghiotto, ${ }^{l}$ E. Guaschino, ${ }^{I} M$. Loi, ${ }^{I} N$. Arce Leal, ${ }^{1}$ F. Balsamo, ${ }^{2}$ R. Manni, ${ }^{1,3}$ G. Nappi

${ }^{l}$ Headache Unit, University Centre for Adaptive Disorders and Headache (UCADH), Pavia, Italy; ${ }^{2}$ Unit of Sleep Medicine and Epilepsy, IRCCS “C. Mondino" Institute of Neurology Foundation, Pavia, Italy; ${ }^{3}$ Department of Clinical Neurology and ORL, University of Rome "La Sapienza”, Rome, Italy; e-mail: grazia.sances@mondino.it

Migraine and epilepsy are paroxysmal disorders showing clinical and epidemiological comorbidity whose possible relationship remains to be clarified. When a seizure begins during or close to post-aura symp- toms, we refer to migralepsy. This phenomenon is described in subjects suffering from migraine with aura; the ICHD-II (2004) diagnostic criteria codify this new entity at 1.5.5 (in the chapter on migraine). A 36year-old man was referred to our headache centre with aura symptoms. The patient's history (birth, physiological history and psycho-physical development) was normal. His history of migraine (without aura and showing a weekend pattern) started at age 20 . At 34 , he began experiencing frequent visual bilateral symptoms lasting 10 minutes and never followed by headache (12 episodes/month). These occurred, in particular, in the evening, after work. He described flashing lights, diffuse in the visual hemifield on the headache side, lasting 5-10 minutes. He also recalled two episodes of dejà-vu symptoms associated with nausea. At age 36, he reported an episode of his typical visual symptoms associated with a dejà-vu perception, perception of a "clean" smell (of fresh laundry), and nausea, lasting about 10 minutes. He then lost consciousness, fell to the ground, and displayed tonic seizure of the limbs with laceration of the tongue. After about five minutes, he resumed normal respiration, but remained rather unresponsive and confused until the arrival of rescue workers. Apparently, this episode was not followed by headache. MRI and basal EEG were normal, whereas sleep deprivation EEG showed a diffuse and large theta frequency in the left cerebral hemisphere. The diagnosis was tonic seizure in migraine with/without aura. The patient began treatment with topiramate (200 mg daily).

This patient was referred to us with recurrent migraine attacks and the appearance of visual symptoms sometimes followed by headache. We investigated possible comorbidities of the aura: patent foramen ovale was excluded by transcranial Doppler; we found heterozygous C677T mutation in the methylene tetrahydrofolate reductase gene. We prescribed acetylsalicylic acid (100 mg daily).

At follow-up (the most recent was three years after the first visit), he reported a reduction of aura symptoms (about five episodes/month) not always followed by headache and no migraine attack without aura. No other seizure occurred and the most recent EEG was normal.

Migralepsy is a rare form with very few reported cases. Even in the cases that have been described, the diagnosis is not clear-cut. The difficulty of arriving at a precise differential diagnosis of visual disturbance (aura vs. occipital partial seizures and elementary hallucinations) does not support the hypothesis that migralepsy is a primary entity in its own right.

\section{ACUTE MANIA INDUCED DURING A CLUSTER HEADACHE EPISODE: A CASE REPORT}

${ }^{1,2} R$. Iannacchero, ${ }^{2}$ M. Gallo, ${ }^{2}$ A. Scardamaglia, ${ }^{2}$ M.A. Massimo, ${ }^{I}$ E. De Caro

${ }^{1}$ Regional Headache Centre, "Pugliese-Ciaccio" Hospital, Catanzaro, Italy; ${ }^{2}$ Department of Mental Health, A.S. 6, Lamezia Terme, Italy; e-mail: rosarioiann@ tiscali.it

Background Cluster headache often leads to mood changes in patients, in particular, to depressive reactions and sometimes to suicide. However, there are no controlled studies on the comorbidity of cluster headache and affective disorders such as manic-depressive illness. We report on a patient who developed his first manic episode while suffering from a cluster episode.

Case report A 34-year-old male patient has been suffering from episodic cluster headache according to IHS criteria for 8 years. He experienced one cluster episode per year with a duration of about 2 months. The left-sided cluster attacks occurred about 4 times a day with a single duration of about half an hour. Oxygen inhalation, and subcutaneous sumatriptan promptly aborted an attack. In recent episodes, verapamil at a dosage of 360 and $480 \mathrm{mg}$ per day was effective as prophylactic agent. At the beginning of his last cluster episode, he received steroids from his general practitioner at a daily dosage of $125 \mathrm{mg}$ per week, which was progressively decreased. At first the attacks stopped, but then recurred after 10 days; thus the patient was 
admitted to our Headache Centre. However, a few days later, while still under steroids, the patient rapidly developed manic symptoms and had to be admitted to a psychiatric isolation ward. Treatment with quetiapine 300-600 mg per day was started, and after 10 days the manic symptoms disappeared; cluster attacks occurred occasionally under this monotherapy.

Conclusions To our knowledge, this is the first report on the onset of manic symptoms during a cluster episode. Since the patient had some endogenous depressive episodes in the past, the diagnosis of manicdepressive illness was made according to the International Classification of Disease, 10th edition (ICD-10). The manic episodes were probably induced by steroids or by steroid withdrawal. We conclude that cluster headache patients with a history of affective diseases should not be treated with steroids but with quetiapine or lithium, which are effective in both conditions.

\section{HYPNIC HEADACHE WITH A SPORADIC PATTERN}

${ }^{1}$ F. Mainardi, ${ }^{2}$ C. Lisotto, ${ }^{3}$ F. Maggioni, ${ }^{1}$ F. Paladin, ${ }^{3}$ G. Zanchin ${ }^{1}$ Headache Centre, Neurological Unit, SS Giovanni and Paolo Hospital, Venice, Italy; ${ }^{2}$ Headache Unit, San Vito al Tagliamento Hospital, San Vito al Tagliamento, Pordenone, Italy; ${ }^{3}$ Headache Centre, Department of Neurosciences, University of Padua, Padua, Italy; e-mail: fmainardi@iol.it

Hypnic headache $(\mathrm{HH})$ is a primary headache disorder, classified in the fourth chapter of ICHD-II, which occurs exclusively during sleep and usually begins after the age of 60 years [1]. The natural evolution of $\mathrm{HH}$ is not well known, but it is assumed that it tends to be chronic unremitting. A follow-up of at least 2 years is described for only 25 patients among the 85 cases reported in the literature; according to the outcome data obtained from these patients, we recently proposed to distinguish between chronic and episodic $\mathrm{HH}$ [2].

A 63-year-old woman complained of a dull, severe headache in the vertex, which developed only during sleep and awakened the patient, without any accompanying signs or symptoms; the headache completely resolved 30 minutes after awakening. She referred the first episode two years before our visit and its recurrence with a very low rate (one attack every six months, for a total of four episodes, the last one just before the observation). Due to the short duration of the attack, the patient did not take any drug. A detailed interview revealed the presence of two more forms of primary headache (migraine without aura and infrequent episodic tension-type headache). General and neurological examinations, laboratory tests, and brain MRI with gadolinium were normal.

To our knowledge, this is the first case of a headache fulfilling ICHDII criteria for $\mathrm{HH}$ with an infrequent pattern, and it supports our previous observations regarding the possibility of an episodic evolution of this rare primary headache.

\section{References}

1. Lisotto C, Maggioni F, Zanchin G (1999) Sindrome della cefalea ipnica: prima descrizione di un caso italiano. In: Gallai $\mathrm{V}$ et al (eds) Le cefalee verso il terzo millennio. Atti del XIV Congresso Nazionale SISC, Perugia pp 277-279

2. Lisotto C, Mainardi F, Maggioni F, Zanchin G (2004) Episodic hypnic headache? Cephalalgia 24:681-685

\section{PRIMARY STABBING HEADACHE IN THE ELDERLY: A CASE REPORT}

${ }^{I}$ F. Mainardi, ${ }^{l} F$. Dainese, ${ }^{2}$ P. Pagan, ${ }^{3} C$. Palestini, ${ }^{1} F$. Paladin, ${ }^{4}$ G. Zanchin

${ }^{1}$ Headache Centre, Neurological Unit, ${ }^{2}$ Radiology Department, SS Giovanni and Paolo Hospital, Venice, Italy; ${ }^{3}$ Department of Medicine, Adria Hospital, Adria, Rovigo; Italy; ${ }^{4}$ Headache Centre, Department of Neurosciences, University of Padua, Padua, Italy; e-mail: fmainardi@iol.it
We present the case of a 74-year-old man referred because of the onset in the previous month of a severe head pain occurring as a series of stabs (generally 3-5) in the left parietal area, lasting up to two seconds and recurring with irregular frequency without any accompanying signs or symptoms. He never experienced a recurrent headache before. The pain occurred every hour during the morning, and two or three times in the afternoon. The stabs presented with an average frequency of four days per week. His medical history revealed the presence of ischemic heart disease with atrial fibrillation and hypertension. Because of his heart condition, a cardiac pacemaker was implanted and he regularly took anticoagulants and hypotensive drugs. The general and neurological investigations were normal. A brain CT scan with contrast medium revealed no alteration; it was not possible to perform a brain MRI because of the presence of the heart pacemaker. We suggested indomethacin at the dose of $50 \mathrm{mg}$ daily. From the second day of therapy the stabs disappeared. He is still headache-free after 3 months of follow-up.

Primary stabbing headache is classified in ICHD-II within chapter 4 "Other Primary Headaches". The short-lasting duration and the irregularity of its frequency justify the low rate of consultation, despite the relative diffusion of the condition, which occurs in $35 \%$ of the general population, according to recent data. In the same population-based study, the peak was reached in the third decade; the onset in the elderly is rarely reported [1].

The peculiarities of our case are: i) the sudden onset in the elderly; ii) the prompt response to low indomethacin dose. Further studies are warranted to better specify the diagnostic criteria for this form of primary headache and its treatment.

\section{Reference}

1. Sjaastad O, Pettersen H, Bakketeig LS (2001) The Vaga study; epidemiology of headache I: the prevalence of ultrashort paroxysms. Cephalalgia 21:207-215

\section{A MIGRAINE-LIKE HEADACHE}

${ }^{1}$ F. Mainardi, ${ }^{2}$ M. Trucco, ${ }^{1}$ A. Campioni, ${ }^{3}$ F. Maggioni, ${ }^{l} F$. Paladin, ${ }^{3}$ G. Zanchin

${ }^{1}$ Headache Centre, Neurological Unit, SS Giovanni and Paolo Hospital, Venice, Italy; ${ }^{2}$ Headache Unit, Neurological Division, Santa Corona Hospital, Pietra Ligure, Italy; ${ }^{3}$ Headache Centre, Department of Neurosciences, University of Padua,Padua, Italy; e-mail: fmainardi@iol.it

The cornerstone of migraine diagnosis, in the absence of pathognomonic biological or imaging markers, are the anamnestic data, with particular regard to the clinical features of the headache, and the general and neurological examinations. Neuroimaging or laboratory tests are useful to exclude a secondary origin, when: $i$. the characteristics of the headache do not fulfil ICHD-II criteria for the diagnosis of migraine; $i i$. the general or neurological examinations are abnormal; iii. the anamnestic data show the presence of alarm features, the so-called red flags.

A 37-year-old woman presented with a one-month history of daily, severe, diffuse headache with phonophobia, photophobia, nausea, and vomiting. She attributed these symptoms to migraine, a condition she suffered from since her twenties with an average frequency of 2 attacks per month. She decided to undergo a medical consultation because of the lack of response to the usual therapy (triptans and NSAIDs) and the progressive worsening of the symptoms. General and neurological examinations did not reveal any abnormalities. A brain CT scan showed the presence of several metastases in both hemispheres with a dislocation of the mid-line structures. Further investigations demonstrated a primary ovarian neoplasm. The patient died two months later. The possibility of a secondary origin was suggested by the accelerating pattern of a previous well-known headache and the lack of response to treatment, both of them to be considered as red flags. The peculiarity of this case is essentially represented by the normal general and neurological examinations despite the dramatic neuroimaging picture. 
Secondary headaches fulfilling ICHD-II criteria for the diagnosis of a primary headache are described also for other forms (e.g. cluster headache, TACs) [1-2]. The adherence to major TACs ICHD-II criteria and a normal general and neurological visit are necessary, but not sufficient to exclude a secondary process. Thus, the appearance of a "red flag" in a headache patient should always prompt a re-evaluation to exclude the presence of a secondary cause.

\section{References}

1. Mainardi F, Maggioni F, Dainese F et al (2002) Spontaneous carotid artery dissection with cluster-like headache. Cephalalgia 22:557-559

2. Trucco M, Mainardi F, Maggioni F et al (2004) Chronic paroxysmal hemicrania, hemicrania continua and SUNCT syndrome in association with other pathologies: a review. Cephalalgia 24(3):173-184

\section{TRIPTAN OVERUSE AND EPISODIC SEROTONIN SYN- DROME \\ ${ }^{1}$ F. Mainardi, ${ }^{2}$ A. Palmieri, ${ }^{1}$ F. Dainese, ${ }^{3}$ C. Lisotto, ${ }^{1}$ F. Paladin, ${ }^{4} G$. Zanchin \\ ${ }^{1}$ Headache Centre, Neurological Unit, SS Giovanni and Paolo Hospital, Venice, Italy; ${ }^{2}$ Headache Unit, Service of Neurology, Hospital of San Donà di Piave, San Donà di Piave, Venice, Italy; ${ }^{3}$ Headache Unit, San Vito al Tagliamento Hospital, San Vito al Tagliamento, Pordenone, Italy; ${ }^{4}$ Headache Centre, Department of Neurosciences, University of Padua, Padua, Italy; e-mail: fmainardi@iol.it}

Serotonin syndrome (SS) is a rare pathological condition related to the concomitant use of drugs that increase 5-HT availability; the pathophysiology of SS seems to be ascribed to the stimulation in the brainstem of $5-\mathrm{HT}_{1 \mathrm{~A}}$ receptors and the inhibition of $5-\mathrm{HT}_{1 \mathrm{~A}}$ dopaminergic transmission, secondary to serotonergic hyperactivity. SS includes several neurological and autonomic manifestations that follow administration or an increased dosage of serotonergic drugs, and is clinically defined in the presence of at least three of the following signs/symptoms: mental status changes, myoclonus, hyperreflexia, tremor, fever, diaphoresis, shivering, and diarrhoea [1]. Because of their actions, triptans have been implicated in the pathogenesis of SS, in particular, if taken with drugs which increase 5-HT levels. We describe the case of a 61-year-old woman, who presented at a first visit a headache fulfilling ICHD-II criteria for probable chronic migraine without aura and probable triptan overuse headache. She treated her attacks with triptans, taking an average 29-30 doses per month for at least 6 months; sometimes (once a week) she had to take triptans twice daily. Furthermore, she took mirtazapine $30 \mathrm{mg} /$ day for a depressive syndrome. She referred episodes of intense sweating, mental confusion, shiver, tremor, and inconstant diarrhoea, lasting 3-4 hours and leaving her prostrate. She complained of such episodes a few hours after taking a second dose of triptan. She first reported these symptoms five months before our observation, but she attributed them to her depressive status. Symptoms disappeared after the withdrawal of mirtazapine and a detoxifying treatment (follow-up 3 months), whereas the headache frequency remained unchanged; therefore, the final diagnosis was chronic migraine without aura.

The peculiarities of this case are: $i$. the temporal pattern and the spontaneous remission of each episode, indicating a recurrent and mild form of SS; $i i$. the correlation between taking a double dose of triptans and the onset of SS symptoms, documented by a diary; iii. the concomitant use of mirtazapine, a drug rarely implicated in generating this syndrome.

Reference

1. Boyer EW, Shannon M (2005) The serotonin syndrome. N Eng J Med 352:1112-1120

\section{CONTRIBUTION OF NEUROBIOLOGICAL AND PSYCHO- LOGICAL TRAITS IN MEDICATION-OVERUSE HEADACHE (MOH) DEVELOPMENT: A CASE REPORT}

${ }^{1} V$. Rispoli, ${ }^{2}$ R. Iannacchero, ${ }^{2}$ E. De Caro
${ }^{I}$ Department of Pharmacobiological Science University "Magna Grcecia" of Catanzaro, Catanzaro, Italy; ${ }^{2}$ Regional Headache Centre, "Pugliese-Ciaccio" Hospital, Catanzaro, Italy; e-mail: rispoli@unicz.it

Even though it is not yet clear whether the misuse and/or abuse of analgesics or triptans in migraine may be involved in headache chronicity, chronic pain and pain relief are considered the most important features in inducing a habitual behaviour as therapeutic need. It is well known that complex brain areas, as well as different neurotransmitter systems and complex responses participate in the biology of the reward brain system. Comorbidity for psychiatric disorders, such as anxiety and depression, as well as negative life events, exacerbate headache and quality of life in patients suffering from headache [1]. Stressful periods, can induce disruption in these interneural interactions and result in a craving for drugs to relieve the pain and bad feelings. Serotonin plays an important role in migraine pathogenesis, and drugs such as triptans, 5-HT $1 \mathrm{~B} / 1 \mathrm{D}$ agonists, are the first choice for acute migraine therapy. Recent findings support the hypothesis that serotonin could contribute to the development of mechanisms underlying the reward system in the brain, inducing a compulsive habit for consumption of antimigraine analgesics [2]. The present paper would like to contribute to clarifying the above described mechanisms. For this purpose, a case report is introduced and discussed.

A 45-year-old woman complained of primary migraine for many years. The headache occurred two, sometimes three times a week. Prodromic symptoms, such as tension in the orbital region and nausea, preceded the headache. For about two years, the patient abused of analgesics (NSAIDs), and during the last year she took triptans (9 to 10 times a month during the last three months) and analgesics. Despite the drug treatment, headache recurred more frequently, and the characteristics of the pain changed, from being sharp and starting abruptly to often presenting in one hemiscalp and spreading subsequently to the entire head. The clinical story of the patient revealed psychiatric co-morbid disorders for anxiety and depression following a stressful period for jobrelated problems (mobbing?). A genetic study was carried out to investigate inherited predisposition to thrombophilia and verify the possibility of sinus venous thrombosis. A Factor $\mathrm{V}$ gene mutation was found. Moreover, neuroimaging investigations (MRI and intracranial venous MR angiography) did not show any pathological brain conditions but revealed a dominant dilated and tortuous right transverse sinus, whereas the left transverse sinus was hypoplastic. During hospitalization, drug treatment was stopped and prophylaptic therapy was started.

In conclusion, this case report suggests that headache chronicity might be caused by biological as well as psychological traits. Correct management of migraine and headaches may contribute to modifying habitual behaviour and reducing consumption of drugs in chronic sufferers. References

1. Silberstein SD, Lipton RB, Breslau N (1995) Migraine: association with personality characteristics and psychopathology. Cephalalgia 15:358-369

2. Fletcher PJ, Korth KM (1999) Activation of 5-HT1B receptors in the nucleus accumbens reduces amphetamine-induced enhancement of responding for conditioned reward. Psychopharmacology $142: 165-174$

\section{A CASE OF UNSATISFACTORY HEADACHE MANAGEMENT SUPPORTING THE NEED FOR INFORMATION AND EDUCA- TION ABOUT HEADACHE DISORDERS}

E. Tasciotti, B. Cappa, D. Camaioni, M. Evangelista

Institute of Anaesthetics, Intensive Care and Pain Therapy, Catholic University "Sacro Cuore", Rome, Italy; e-mail: edotasciotti@ hotmail.com

Introduction With this case report we want to emphasize how important it is, and how much work still needs to be carried out in terms of patient education and divulgation of information about headaches. 
Case A 41-year-old man suffered from headache (the first episode occurred about 15 years ago). This affection became worse in frequency, intensity and refractory to the rescue dose in the last 12 months, compromising his job. The characteristics of this headache were: daily frequency; right-sided localization, beginning in the posterior region of the head; absence of aura except for tension located in the cervico-occipital area over the paravertebral muscles, associated with pain in the point of emergency of Arnold's nerve; bilaterally lasting $\geq 24$ hours. Pain intensity was measured with Visual Analogue Scale (VAS). Phonophobia and photophobia were so strong during the crises that they forced the patient to abandon his activity. Under prescription, the patient had taken $500 \mathrm{mg}$ effervescent tablet as rescue dose. Consecutively, because the headaches did not resolve, he took $10 \mathrm{mg}$ of rizatriptan RPD, which he defined has being unsatisfactory for pain relief. The patient did not receive any information about the rescue dose nor prophylactic drugs from the previous doctors, even though he had been suffering from headache for the past 15 years and was experiencing increased headache frequency.

The patient underwent algological examination: finger pressure caused pain at the paravertebral cervical muscles conserving flex extension and laterality of the first trigeminal branch. The trigger point was on the right trapezoidal muscle.

Chronic sinusitis was found in the patient, who worked in an area subject to climatic variations. He was allergic to acetylsalicylic acid. Instrumental diagnosis, $x$-rays of the head and cervical spine in two separate moments (23/02/06), showed a reduction of physiological cervical lordosis, which resulted in osteoarthritis. The patient was prescribed the following therapy:

Fluxarten $5 \mathrm{mg}$ at 9 p.m.

Laroxyl $5 \mathrm{mg}$ gtt at 9 p.m.

Sirdalud $1 / 2$ tablet bid

Rizatriptan RPD 10 mg s.l., when needed.

Therapeutic efficacy was and is still being evaluated monthly by VAS. Results The described therapy was followed continuously considering the high tolerability of the prophylactic drugs. After 4 weeks, the monthly frequency of crisis decreased to three, with $\mathrm{VAS}=7$ and the patient responded to rizatriptan taken at the beginning of the crisis. After 8 weeks, the frequency decreased to 2 crises, VAS $=5$, and the patient responded to rescue dose. Currently, the patient is drug free and refers two headache crises per month. He has returned to work and now considers his social life normal.

Conclusions This case represents the paradigm of an incorrect treatment for headache symptoms, regarding both the most suitable strategy to use and when to take the symptomatic drug. It is evident how a symptomatic strategy for daily headache exposed the patient to drug overuse and to an unsuccessful therapy causing social and working problems. It should be mentioned that the lack/absence of correct information regarding early prophylaxis also contributed to the unsuccessful resolution of headache pain.

\section{HEMIPLEGIC MIGRAINE WITH PROLONGED SYMPTOMS IN ATYPICAL STURGE-WEBER SYNDROME}

M.A. Tavoni, N. Zamponi, E. Cesaroni, L. Porfiri, C. Cardinali

Riuniti University Hospitals, Paediatric Neurology Department, Children's Hospital “G. Salesi”, Ancona, Italy; e-mail: npisalesi@ ao-salesi.marche.it

Introduction The Sturge-Weber Syndrome (SWS) is a rare disease characterized by an encephalotrigeminal or leptomeningeal angiomatosis and facial vascular nevus (port-wine stain). It is part of a phenotypic spectrum, which includes other syndromes with capillary angiomatosis malformations in various sites, such as Klippel Trenaunay Weber Syndrome with malformations not only of the capillaries, veins, arteries, and lymphatic vessels, but also of soft tissues and bone hypertrophy [1].

Case report We report a patient with a mixed phenotype, of a facial nevus on the right forehead, right arm and left leg soft tissues and bone hypertrophy, obesity, high blood pressure, and epileptic seizures, who presented at 14-years of age with pulsating unilateral headache with visual disorders and acute - prolonged left hemiparesis. None of the episodes were preceded or accompanied by loss of consciousness or ictal clonic jerks. The EEG was normal during the headache episode, MRI showed the typical Sturge-Weber angiomatosis without ischemic lesions.

Results and discussion Our case report suggests a Sturge-Weber atypical syndrome that could be responsible for symptomatic migraine-like headache. According to criteria of the ICHD-II classification 2004, this syndrome could be clinically diagnosed with probable migraine with aura (subtype 1.6.2) with atypical features.

Vasomotor modifications in venous flow in relation with pial angiomatosis are probably at the origin of these migraine-like episodes. Chronic oligoemia and the pre-existent metabolic cortex damage caused by hypoperfusion would worsen the clinical symptomatology [2].

Epileptic seizures between the prodromic and pain phase of migraine attack are rarely reported in paediatric age and in patients affected by SWS. Probably, the migraine attacks, like the seizures, are to be considered another expression of brain damage as a result of microvascular disturbances.

\section{References}

1. Chabriat H, Pappata S, Traykov L et al (1996) [Sturge-Weber angiomatosis responsible for hemiplegia without cerebral infarction in term pregnancy] Rev Neurol 152:536-541 [Article in French]

2. Lisotto C, Mainardi F, Maggioni F, Zanchin G (2004) Headache in Sturge-Weber Syndrome: a case report and review of the literature. Cephalalgia 24:1001-1002

\section{A CASE OF POSTERIOR SCLERITIS: HEADACHE ATTRIB- UTED TO OCULAR INFLAMMATORY DISORDER}

${ }^{l}$ S. Ruffatti, ${ }^{l}$ L. Santelli, ${ }^{I} F$. Maggioni, ${ }^{I}$ E. Mampreso, ${ }^{2} F$. Dainese, ${ }^{I}$ G. Zanchin

${ }^{1}$ Headache Centre, Department of Neuroscience, University of Padua, Padua, Italy; ${ }^{2}$ Headache Centre, Neurological Unit, SS Giovanni and Paolo Hospital Venice, Italy; e-mail: silvia.ruffatti@unipd.it

Introduction A case of posterior scleritis is described. Posterior scleritis is an uncommon form of scleral inflammation, which can be confused with several inflammatory and non-inflammatory ocular diseases or ocular tumors. Anterior scleritis can be associated with this condition. It may be idiopathic or associated with systemic disease, particularly connective tissue disorders [1]. In the International Headache Society Classification [2] it is included in the chapter "Headache attributed to ocular inflammatory disorder" (11.3.4). Periocular pain, pain on movement and decreased vision are the most frequent symptoms [1]. Pain can also be referred to the forehead, maxilla, or sinuses and is described as penetrating in character. Redness of the affected eye can be observed. Ultrasonography is the key investigation necessary to make the diagnosis of posterior scleritis [1]. CT scan and fundus fluorescein angiography can also be used.

Case history We report a case of a 26-year-old woman who began to complain of right-sided ocular and periocular pain, exacerbated by ocular movements, irradiating to the ipsilateral temple and zygoma, not associated with visual disturbances. Admitted to our Emergency Department, she underwent an ophthalmological consultation, which showed a normal fundus oculi and visual acuity. Trigeminal neuralgia was suspected and she was then treated unsuccessfully with analgesics. Seven days later, she underwent a second ophthalmological and a neurological consultation, which were both normal. After one week, she experienced a worsening of facial pain associated with conjunctival injection, eyelid edema, and developed a central scotoma. She was then admitted to our clinic. Fundus oculi showed in the right eye a hyperemic optic disc with blurred margins. Blurred vision in the right eye with a central scotoma was referred. The remaining neurological examination was normal. A third ophthalmological consultation showed a visual acuity of $1 / 10$ in the right eye (10/10 in the left) with conjuncti- 
val injection. Fundus oculi confirmed the above reported findings and some retinal folds; ultrasonography revealed scleral thickening and exudative retinal detachment, suggesting a posterior scleritis. Chest Xray, routine blood tests, brain MRI with gadolinium and cerebrospinal fluid examination were normal.

The patient was treated with methylprednisolone i.v. $1 \mathrm{gr} / \mathrm{day}$ for 10 days with complete regression of pain and clear improvement of visual disturbances. Ultrasonography performed 10 days after the end of treatment showed a reduction of scleral thickening, fluid in the Tenon capsule, and regression of retinal detachment. Visual acuity was $6 / 10$ in the right eye. A month after discharge the patient began to complain of left periocular pain, without visual loss. Ultrasonography showed posterior scleritis in the left eye; she fully recovered after a further course of steroid treatment.

Discussion Posterior scleritis is a common form of scleritis and should be suspected in patients who present with ocular pain and visual loss. It is one of the most commonly misdiagnosed conditions in ophthalmology, especially when, as in our case, pain is not associated with visual disturbances or ocular signs at the onset of symptoms.

References

1. McCluskey PJ, Watson PG, Lightman S et al (1999) Posterior Scleritis: clinical features, systemic associations, and outcome in a large series of patients. Ophthalmology 106:2380-2386

2. Headache Classification Subcommitee of the International Headache Society (2004) The International Classification of Headache Disorders, 2nd edn. Cephalalgia 24:[Suppl 1]:9-160

\section{HEADACHE AND ISOLATED PARALYSIS OF THE 12TH CRANIAL NERVE AS A PRESENTING SYMPTOM OF DIS- SECTION OF THE INTRAPETROSAL TRACT OF THE INTER- NAL CAROTID ARTERY}

\section{S. Tenaglia, A. Baldi, P. Sarchielli, P. Calabresi}

Headache Centre, Neurologic Clinic, Department of Medical Surgical Specialties and Public Health, University of Perugia, Perugia, Italy; e-mail: baldianto@libero.it

Case description A 60-year-old man came to our attention for the onset of symptomatology characterized by continuous, left-sided, fronto-orbital headache of moderate intensity, associated with photophobia, which did not regress after taking common analgesics. After about three days, the patient presented with a worsening of the headache, which was of stronger intensity and with the appearance of dysphagia and dysarthria.

The neurological examination revealed a deficit of the left XIIth cranial nerve. Brain CT, brain MRI, Echo-colordoppler of the supra-aortic vessels, and ultrasound of the neck were normal. Angio-CT and subsequent angio-MRI of the vessels of the neck showed findings compatible with dissection of the intrapetrosal segment of the left internal carotid artery.

Discussion Carotid artery dissection is an important cause of stroke in the young. Headache and facial pain, often associated with Horner's syndrome, constitute a common onset symptom and may precede stroke by a few days [1]. Headache associated with deficit of the hypoglossal nerve is not a common onset symptom, and only a few cases are described in the literature [2]. More frequently, this symptomatology may be secondary to diseases of the jugular foramen, and in this case, is associated with paralysis of other cranial nerves ( $\mathrm{IX}^{\mathrm{th}}$ and $\left.\mathrm{X}^{\mathrm{th}}\right)$.

In the case described, brain MRI excluded intracranial diseases. EchocolorDoppler of the supra-aortic vessels could not visualise the dissection, in that this involved the intraosseous segment of the internal carotid artery, thus warranting a specific study of the complete intracranial course of this artery.

References

1. Guillon B, Levy C, Bousser MG (1998) Internal carotid artery dissection: an update. J Neurol Sci 153:146-158
2. Knibb J, Lenthall R, Bajaj N (2005) Internal carotid artery dissection presenting with ipsilateral tenth and twelfth nerve palsies and apparent mass lesion on MRI. Br J Radiol 78:659-661

\section{PAINFUL RECURRENT BILATERAL OPHTHALMOPLEGIA SECONDARY TO BILATERAL CAROTID CAVERNOUS FISTULA}

A. Baldi, F. Coppola, C. Rossi, P. Sarchielli, P. Calabresi

Neurologic Clinic, Department of Medical Surgical Specialties and Public Health, University of Perugia, Perugia, Italy; e-mail: baldianto@libero.it

Case description A 68-year-old woman presented to us one week after onset of symptoms characterized by a stabbing headache of strong intensity, in the retro-orbital and left fronto-temporal regions, with nausea, photophobia and phonophobia associated with diplopia. The neurological examination revealed paresis of the left VIth cranial nerve, tactile hypoesthesia, and left-sided pain of the first division of the trigeminal nerve.

Brain CT revealed slight enhancement with gadolinium in the left cavernous sinus. Blood tests, Echo-colorDoppler ultrasound of the supra-aortic vessels and the superficial temporal arteries were normal. The patient was treated with prednisone $50 \mathrm{mg} /$ day for one week and subsequently $25 \mathrm{mg} / \mathrm{day}$. After 48 hours the headache regressed, but the diplopia persisted.

After two months, the patient also presented diplopia on right gaze, eyelid edema, conjunctival injection in the right eye, associated with unilateral retro-orbital pain. The neurological examination revealed: proptosis, eyelid edema, conjunctival chemosis, partial deficit of the right IIIrd cranial nerve, and deficit of the left VIth cranial nerve.

Brain MRI was repeated and in the norm. Subsequently, brain angioMRI was performed, which revealed a bilateral dural fistula of the cavernous sinuses and the occiptal foramen.

The lesion was treated with embolization via the left transjugular approach.

Discussion Dural arteriovenous fistulas (AVFs) are a rare condition, with a rate of incidence of 0.16 per 100000 inhabitants/year; with respect to other intracranial vascular malformations they can be acquired, often as a consequence of cerebral venous thrombosis, previous head traumas, aneurysmal rupture, and local and systemic infections [1]. Cavernous carotid fistulas constitute $20 \%-40 \%$ of all dural AVFs [2], and are formed by an abnormal communication between the carotid artery and cavernous sinus.

Venous hypertension subsequent to shunt formation results in retrograde venous drainage into the ophthalmic venous system, which is responsible for the ocular symptoms, cortical venous reflux, and tendency to haemorrhage.

Headache is rarely a presenting symptom, but when present, it is associated with ophthalmoplegia. The differential diagnosis is with other forms of painful ophthalmoplegia.

Neuroimaging studies are normal or furnish indirect signs (often non-specific) of fistula presence; the only certain diagnostic investigation is brain angio-MRI [1].

In the case of carotid-cavernous fistulas, among the various therapeutic options endovascular embolization is the least invasive method, and when possibile, the transvenous access can lead to complete obliteration of the fistula [1].

References

1. Brown RD (1999) Epidemiology and natural history of vascular malformations of the central nervous system. In: Jafar JJ, Rosenwasser RH, Awad IA (1999) Vascular Malformations of the Central Nervous System. Lippincott Williams \& Wilkins, Philadelphia, pp 129-148

2. Kiyosue H, Hori Y, Okahara M et al (2004) Treatment of intracranial dural arteriovenous fistulas: current strategies based on location and hemodynamics, and alternative techniques of transcatheter embolization. Radiographics 24:1637-1653 


\section{PAINFUL OPHTHALMOPLEGIA WITH CSF ALTERATIONS: A CASE REPORT}

A. Mercurio, G. Caranci, M. Altieri, V. Di Piero

Headache Centre, Department of Neurological Sciences, University of Rome "La Sapienza”, Rome, Italy; e-mail: vittorio.dipiero@uniromal.it

Introduction Painful ophthalmoplegia (PO) is a syndrome characterized by the occurrence of periorbital pain and ophthalmoparesis, with or without the involvement of other cranial nerves, caused by several different etiologies.

Case historty A 46-year-old man was admitted to our ward, with a severe dull and steady pain in the right orbit and in the surrounding area of the forehead, associated a few days later with the appearance of diplopia and right eyelid ptosis. The neurological examinations showed a paresis of both the right third and fourth cranial nerves. Physical and laboratory examinations, trigeminal reflexes, cerebral MRI, arterial and venous MR angiography were all normal.

A CSF sample showed an elevated protein content $(93 \mathrm{mg} / \mathrm{dL})$, with a positive Pandy reaction and alteration of blood-brain barrier indices, and was negative for the presence of common infectious agents. Two days after the clinical admission, the headache disappeared, and the third and fourth nerve palsy improved significantly, with full recovery from diplopia a few days later. The increase in CSF proteins persisted at 2 weeks $(73 \mathrm{mg} / \mathrm{dL})$ and 1 month $(64 \mathrm{mg} / \mathrm{dL})$.

There are several causes of PO: vascular, inflammatory, infections, dismetabolisms, neoplasia, trauma, recurrent demyelinating neuropathy and ophthalmoplegic migraine (OM). OM may be suspected when a severe headache is associated with ophthalmoparesis, normal MRI investigations and a benign course [1]. In our case this diagnosis could be hypothesized. In this regard, it is noteworthy that the IHS criteria (1988) required a single episode (probably because of its rarity), while ICHD-II criteria require its recurrence [1]. In our patient, the CSF abnormalities, the single episode and the "nonmigrainous" features of the headache, except for the severity, are consistent with an uncommon viral infection. Because $\mathrm{OM}$ is more a syndrome than a specific disease, this case suggests that if a diagnosis of OM is suspected, the possibility of a benign inflammatory disease should be considered, in particular, when ICHD-II criteria are not fully satisfied [1].

Reference

1. Headache Classification Subcommittee of the International

Headache Society (2004) The International Classification of Headache Disorders, 2nd edn. Cephalalgia 24[Suppl 1]:9-160

\section{HEADACHE IN A PATIENT WITH CEREBRAL SINUS THROMBOSIS AND SPONTANEOUS INTRACRANIAL HYPOTENSION}

${ }^{1}$ E. Mampreso, ${ }^{I}$ F. Maggioni, ${ }^{I}$ A.P. Burlina, ${ }^{I}$ C. Perin, ${ }^{2} F$. Mainardi, ${ }^{1}$ G. Zanchin

${ }^{1}$ Headache Centre, Department of Neurosciences, University of Padua, Padua, Italy; ${ }^{2}$ Headache Centre, Neurological Unit, SS Giovanni and Paolo Hospital,Venice, Italy; e-mail: edoardo.mampreso@unipd.it

Cerebral venous sinus thrombosis (CVST) is an uncommon, lifethreatening condition that mostly affects middle-aged women. The principal, and also earliest symptom is headache $(80 \%-90 \%)$, followed by other neurological symptoms. The headache has no specific characteristics, is most often diffuse, progressive, severe and associated with other signs of intracranial hypertension. It can also be unilateral and sudden, and sometimes mimick migraine, primary thunderclap headache, cerebrospinal fluid (CSF) hypotension or subarachnoid haemorrhage (ICHD-II, 2004). CVST recognizes a wide variety of risk factors and causes. In $20 \%-35 \%$ of cases, where aetiology remains unknown, CVST is classified as idiopathic.

Intracranial hypotension (ICH) syndrome is a very infrequent clinical condition. Its most common symptoms are orthostatic headache asso- ciated with nausea and/or vomiting, diplopia, hearing disturbances (hypoacusia, tinnitus), neck pain, visual deficit and vertigo.

We report the case of a 43-year-old female who presented with headache, which began three days before, characterised initially by pressing/tightening, and later on, pulsating quality localised at the vertex, of mild intensity, without accompanying symptoms, associated with mild nausea and tinnitus and occurring only on standing. Brain CT scan showed less evident cortical sulci, small basal cisterns, small ventricular cavities, without parenchymal alterations. Neurological examination showed brisk reflexes and bilateral Babinski sign; fundus oculi was normal. Brain MRI with contrast and angio-MRI showed thrombosis of the transverse and sigmoid sinuses and of the internal jugular vein on the left side, along with alterations suggestive of ICH (pachymeningeal contrast enhancement and Arnold-Chiari-like descent of cerebellar tonsils). The clinical history was unremarkable, in particular for thromboembolic risk factors. Complete blood count, thrombophilia tests, and neoplastic markers were normal, as were ECG, chest X-rays, somatosensory evoked potentials, and epiaortic vessels and transcranial EchoDoppler. Spinal MRI with contrast showed signs of CSF leak (deliquoration) in the dorsal spine. Then, the clinical history was specifically reassessed and no possible causes of CSF leak were identified. The patient was treated with anticoagulant therapy: an angio-MRI performed 18 days later showed a partial recanalization. At discharge and at two months follow-up the patient was asymptomatic.

Cases of CVST in patients with ICH secondary to CSF leak are found in the literature, but only 4 cases of CVST have been associated with spontaneous ICH. Based on these observations, and on our case, it could be possible that compensatory venous sinus dilation and consequent hematic stasis conditions associated with ICH may predispose to CVST.

MIGRAINE WITH VISUAL AURA ASSOCIATED WITH ARTERIOVENOUS MALFORMATION

${ }^{1}$ G. Alberti, ${ }^{2}$ A. Giorgetti, ${ }^{1}$ G.M. Patruno, ${ }^{1}$ A. Romorini

${ }^{1}$ Neurology Service, Magenta Hospital, Milan, Italy; ${ }^{2}$ Neurology Unit, Neurology Department, Legnano Hospital, Legnano, Milan, Italy; e-mail: alessandro.romorini@ao-legnano.it

Objective To describe two cases of migraine with aura associated with cerebral arteriovenous malformation (AVM).

Case 1: A 57-year-old woman complained of headache of unusual severity. She had a history of migraine since age 17 , characterized by an aura of scintillating scotoma for 20-30 minutes on the right side. Thereafter, a strictly unilateral (left-sided in all cases) throbbing headache with nausea, vomiting and photophobia occurred. Pain was mild and responsive to simple analgesics. MRI showed an arteriovenous malformation in the left occipital lobe.

Case 2: A 22-year-old woman had a history of migraine since age 15 with visual aura of scotoma and fortification (zig-zag patterns) on the left side, occasionally accompanied by numbness in the left hand and face. Her mother had a history of migraine. Three times she had tongue biting; EEG showed epileptiform discharges. She started valproate therapy and the visual aura terminated. The aura always occurred on the left suggesting a possible right-sided structural lesion. MRI showed a right parietal AVM.

Discussion Arteriovenous malformation is a congenital anomaly that consists of abnormal arteries and veins without the presence of a capillary bed. The majority of patients initially show intracranial haemorrhage, while others may present symptoms of seizures, headaches and progressive neurological deficits. In the general population, AVMs are an extremely infrequent cause of headache. Headache occurs in $10 \%-50 \%$ of patients with AVM. The headache may be typical of migraine. Migraine headaches have a complex pathophysiology, and (both vascular and neuronal mechanisms) have been proposed. One possible scenario begins with brain initiated events evolving to cortical spreading depression (CSD). Susceptibility to CSD appears to be genetically determined. Structural lesions may cause the initial event. 
When a patient with migraine manifests symptoms always on the same side, neuroimaging workup is warranted. This should aid in the early diagnosis of cerebral AVM in order to start treatment before the occurrence of cerebral haemorrhage.

\section{TRIGEMINAL NEURALGIA AS PRESENTING SYMPTOM OF ARNOLD CHIARI TYPE 1 MALFORMATION: A CASE REPORT}

G. Caranci, A. Mercurio, M. Altieri, V. Di Piero

Headache Centre, Department of Neurological Sciences, University of Rome "La Sapienza", Rome, Italy; e-mail:vittorio.dipiero@uniroma1.it

Introduction Clinical presentation of Arnold Chiari type 1 malformation is highly variable. About $13 \%$ of patients are asymptomatic headache may be the sole clinical manifestation of the malformation; however, it is one of the most frequent. No headache type seems to be particular to the malformation; both short- and long-lasting headaches are described [1].

Case history A 36-year-old woman presented with throbbing-stabbing pain in the left supraorbital region, which started 3 years earlier. Pain occurred three or four times a day; the paroxystic pain was severe, and lasted 7-10 seconds. There were no warning symptoms; trigger factors were coughing, sneezing, defecation, and nodding movement. There were no accompanying symptoms but mild nausea. Between attacks, she was completely pain-free. She was treated with NSAIDs and carbamazepine without any improvement. Neurological examination and laboratory blood tests were normal. MRI showed an Arnold Chiari type 1 malformation with downward herniation of the cerebellar tonsils through the foramen magnum into the upper cervical spinal canal. A CT scan of the maxillary and frontal sinuses was normal. Neurosurgical intervention was suggested.

Discussion Our patient satisfied all the criteria for a symptomatic neuralgia probably caused by the Arnold Chiari type 1 malformation. In this view, we excluded other types of headaches, such as rhinosinusitis, short-lasting unilateral neuralgiform headache attacks with conjunctival injection and tearing (SUNCT), supraorbital neuralgia and idiopathic trigeminal neuralgia. The otolaryngologic examination and CT scan excluded the possibility of rhinosinusitis.

Due to the location, duration and frequency of the pain described, it could be compatible with a SUNCT, however, the neurovegetative disturbances, such as conjunctival injection and lacrimation, were absent. Regarding the supraorbital neuralgia, it lacked tenderness over the nerve in the supraorbital notch, and there was no pain in the medial aspect of the forehead in the area supplied by the supraorbital nerve. Unlike trigeminal neuralgia, there were no trigger points, a very rare location only in the first branch, and the lack of any response to high carbamazepine dosage [1].

Conclusions To our knowledge, this is the first case with Arnold Chiari Type 1 malformation and symptomatic trigeminal neuralgia exclusively in the first branch. It is probable that the pain is caused by an anatomical compression on the trigeminal nucleus, and that the difference in pressure found in the intracranial and intraspinal compartments, evoked by the Valsalva maneuvre, emphasises this compression, setting off the short-lasting sharp pains [2].

\section{References}

1. Headache Classification Subcommittee of the International Headache Society (2004) The International Classification of Headache Disorders, 2nd edn. Cephalalgia 24[Suppl 1]:9-160

2. Rosetti P, Ben Taib NO, Brotchi J, De Witte O (1999) Arnold Chiari Type I malformation presenting as a trigeminal neuralgia: case report. Neurosurgery 44:1123-1124

\section{HEADACHE ATTRIBUTED TO BRAIN ABSCESS: A CASE REPORT \\ ${ }^{1}$ A. Giorgetti, ${ }^{2}$ M. Conti}

${ }^{1}$ Department of Neurology and ${ }^{2}$ Department of Neurosurgery, Legnano Hospital, Legnano, Milan, Italy; e-mail:andrea.giorgetti@ao-legnano.it

Brain abscess is a focal suppurative process in the brain parenchyma that still contributes to high mortality rates. Outcome is closely related to correct and early management. In order to evaluate early management, we have reviewed the brain abscesses treated in our Department of Neurosurgery during the last five years, choosing to describe one case among these. We present a 38-year-old male who was admitted to the Emergency Department (ED) complaining of a frontal headache and fever $\left(37.5 \mathrm{C}^{\circ}\right)$, unresponsive to treatment with NSAIDs. The patient described weakness and numbness of the right limb, which resolved in 20-30 minutes. After CT scan resulted normal, the patient was discharged. The day after, the patient returned to the ED complaining of two new episodes, characterized by weakness and numbness involving the right limb with spontaneous resolution in ten minutes, while an intense, diffuse bilateral headache persisted. The patient was admitted to our department. The neurological examination was unremarkable. However, within five days the patient showed right hemiparesis; the inflammatory index was elevated and MRI revealed a productive, expanding lesion with marked edema in the left parietal region. The modality of treatment was surgical excision with catheter drainage-aspiration of the left parietal abscess. Outcome was excellent: the patient recovered promptly from the hemiparesis, and there was progressive attenuation of headache until it disappeared. Brain abscesses are severe infections with lethal outcome in the case of delay in diagnosis and treatment. Predisposing factors are sepsis of adjacent organs and bacteraemia. The most frequent clinical manifestations are fever and headache. Headache is usually the most frequently encountered symptom. When under observation our patient continuously complained of bilateral, constant headache with increasing intensity to severe grade, aggravated by straining and accompanied by nausea. A new type of headache associated with general feeling of illness and/or fever should direct attention toward an intracranial infection.

\section{IS THE TREATMENT OF HEADACHE BY SPONTANEOUS CERVICAL CSF LEAK WITH LUMBAR EPIDURAL BLOOD PATCH A RATIONAL CHOICE?}

${ }^{1}$ E. Ferrante, ${ }^{2}$ I. Arpino, ${ }^{I}$ A. Citterio, ${ }^{2}$ A. Savino

${ }^{1}$ Neurosciences and ${ }^{2}$ Emergency Departments, Niguarda Cà Granda Hospital, Milan, Italy; e-mail: enricoferrante@libero.it

Background Spontaneous intracranial hypotension (SIH) generally results from spinal spontaneous CSF leakage [1]. Treatment is usually conservative. Sometimes an autologous EBP at the site of CSF leakage may be necessary [2].

Objective To evaluate the spread of blood injected into the epidural space during lumbar epidural blood patch (LEBP) for treatment of spontaneous cervical CSF leakage (SCCSFL) by MRI.

Materials and methods We report one case of SCCSFL [among the 32 with this condition evaluated between 1992 and the present].

Results A 45-year-old man presented sudden, intense, gravative fronto-occipital orthostatic headache. Neurological examination, routine blood tests, and brain CT scan were normal. Brain MRI showed diffuse pachymeningeal enhancement. Gadolinium spinal MRI showed CSF epidural collection from $\mathrm{C} 2$ to D3. After one month of bed rest, orthostatic headache was still present. The patient was treated with autologous lumbar EBP (25 mL) at the L2-L3 level. The blood was mixed with $0.05 \mathrm{~mL}$ of gadolinium. During and after injection, he remained in the Trendelenburg position (TP) approximately $30^{\circ}$ for 1 hour. Spinal post-patch MRI showed gadolinium mixed with blood in the epidural space from the lumbar to cervical level (C2-C3). He subsequently remained in the TP for 22 hours. He was asymptomatic within 24 hours after LEBP. At 6-month follow-up he was asymptomatic. Discussion and conclusions We demonstrate that EBP, even though injected at the level of the lumbar spine (i.e., far from the site of CSF 
leak), may move upward reaching the cervical segments. This is possibly favoured by the TP. A rapid coagulation response at the level of the dural hole stops CSF leakage. Thus, EBP at the level of the cervical spine, where the procedure is difficult to attempt, may not be necessary. To our knowledge, this is the first report that shows the spread of blood from lumbar to cervical epidural space with LEBP and demonstrates that the treatment of spontaneous cervical CSF leak with autologous lumbar epidural blood patch is a rational choice.

References

1. Ferrante E, Savino A, Sances G, Nappi G (2004) Spontaneous intracranial hypotension syndrome: report of twelve cases. Headache 44:615-622

2. Berroir S, Loisel B, Ducros A et al (2004) Early epidural blood patch in spontaneous intracranial hypotension. Neurology 63:1950-1951

\section{REFERRED FACIAL PAIN AS A SYMPTOM OF NON- METASTATIC LUNG CANCER: A CASE REPORT AND REVIEW OF THE LITERATURE}

A. Palmieri

Headache Unit, Service of Neurology, Hospital of San Donà di Piave, San Donà di Piave, Venice, Italy; e-mail: antonio. palmieri@assl10.veneto.it

Introduction Facial pain is a common clinical symptom caused by a wide variety of underlying conditions, including neurological, odontological, and otolaryngological pathologies. A patient who developed an intractable facial pain as an unusual first manifestation of a remote lung carcinoma is described.

Case Report A 39-year-old woman presented with a 2-month history of pain localized in the left jaw, with extension into the ipsilateral orbit and temple. The pain was continuous, moderate to severe in intensity, and possibly associated with tearing, eye redness, and mild nausea. She had been smoking 1 pack of cigarettes a day, for at least 20 years. An odontological visit, a brain MRI and a carotid Doppler ultrasound were normal. The pain was unresponsive to a number of analgesics as well as carbamazepine, gabapentin, and prednisone. About 2 weeks before presentation the patient developed cough and poor appetite. The neurological examination was unremarkable. The physical examination revealed diminished breath sounds in the left-upper lobe and right supraclavicular lymphadenopathy. A CT scan of the chest showed a large solid mass in the superior aspect of the left mediastinum surrounding the supra-aortic vessels and hilus. A fine-needle biopsy of the supraclavicular node confirmed the presence of a large cell carcinoma. The ESR was markedly elevated at $83 \mathrm{~mm} / \mathrm{h}$. A few days later, the patient became dysphonic, with laryngoscopic evidence of paresis in adduction of the left vocal cord. The facial pain gradually improved and then spontaneously ceased before starting chemotherapy. The patient was still pain-free after a 6 months follow-up.

Discussion Referred facial pain is a rare symptom of nonmetastatic malignant lung lesions, and was first described by Des Prez and Freemon in 1983. Since then, 36 patients (17 M, 19 F) have been described, including the present patient. The presumed mechanism by which a thoracic mass can provoke a pain referred to the face is compression of the vagus nerve, which drives pain signals originating from thoracic and abdominal viscera. Convergence of the visceral and somatic afferents at the level of the descending nucleus of the trigeminal system may explain the ipsilateral referred facial pain. The most frequent locations are the ear (about $90 \%$ of cases), jaw and temple. The diagnosis can be delayed from 1 month to 4 years after the onset of the pain. All patients described were smokers. ESR is increased in most patients. Unlike the previous cases, in the present case the pain spontaneously disappeared after the patient became dysphonic, indicating the putative importance of the integrity, at least in part, of the laryngeal nerve.

Conclusions Lung cancer should be included in the differential diagnosis of facial pain with atypical signs and/or refractory to treatment.

\section{A CASE OF ATYPICAL INTRACTABLE FACIAL PAIN SEC- ONDARY TO METASTATIC LUNG CANCER}

\section{${ }^{I}$ S. Ruffatti, ${ }^{1} \mathrm{~F}$. Maggioni, ${ }^{2} \mathrm{~F}$. Mainardi, ${ }^{2} \mathrm{~F}$. Dainese, ${ }^{3} \mathrm{C}$. Lisotto,} ${ }^{I}$ G. Zanchin

${ }^{1}$ Headache Centre, Department of Neuroscience, University of Padua, Padua, Italy; ${ }^{2}$ Headache Centre, Neurological Unit, SS Giovanni and Paolo Hospital, Venice, Italy; ${ }^{3}$ Headache Unit, San Vito al Tagliamento Hospital, San Vito al Tagliamento, Pordenone, Italy; e-mail: silvia.ruffatti@unipd.it

Introduction Persistent idiopathic facial pain is defined by the International Headache Society Classification (ICHD-II) (chapter 13.18.4) as a pain in the face, present daily and persisting for all or most of the day, confined at onset to a limited area on one side of the face, deep and poorly localised. According to the classification, pain around the ear or temple may precede the detection of an ipsilateral lung carcinoma [1]. Nearly 30 cases of intractable facial pain secondary to lung neoplasm are described in the literature [2]. We report a case of intractable facial pain associated with metastatic lung cancer. Case history A 70-year-old woman presented to the clinic with a 15month history of constant, right-sided facial pain, with paroxysms of the upper teeth, irradiating to the auricular and temporal areas. Six months after the onset of pain she underwent CT scan and EEG, which were both normal. She was unsuccessfully treated with amitriptyline $(40 \mathrm{mg}$ per day) and gabapentin (up to $2000 \mathrm{mg}$ per day). She had additional complaints of poor appetite with weight loss of $4 \mathrm{~kg}$ in the last 6 months and a 9-month history of persistent cough with recent onset of chest pain on the right side. Auscultation of the lungs revealed abnormal breath sounds on the right hemithorax; neurological examination revealed a sensory loss on the right side of the face. Routine blood tests were normal. Chest X-ray revealed a mass in the right hylar region, confirmed by chest CT. She underwent bronchoscopy with lung biopsy; the histological examination revealed pulmonary adenocarcinoma. Brain MRI with gadolinium revealed small multiple supratentorial and infratentorial brain lesions, none of them localized near the right trigeminal nucleus and pathways. The patient was treated with chemotherapy with slight improvement of facial pain. She died shortly thereafter.

Discussion Facial pain secondary to lung cancer is usually described as a unilateral constant ache, ipsilateral to the lung mass, frequently involving the auricular area, and often associated with signs suggesting a lung cancer (weight loss, digital clubbing, cough, etc.). The pain seems to be due to invasion of the vagus nerve [2]. Our case referred to a metastatic lung cancer, but none of the lesions seen on brain MRI could possibly explain the pain referred by the patient and probably occurred at a distance from the onset of pain. It is important to consider lung neoplasm as a possible cause of facial pain, especially if it presents with the characteristics seen above and in smokers.

References

1. Headache Classification Subcommittee of the International Headache Society (2004) The International Classification of Headache Disorders, 2nd edn. Cephalalgia 24:[Suppl 1]:9-160

2. Eross EJ, Dodick DW, Swanson JW, Capobianco DJ (2003) A review of intractable facial pain secondary to underlying lung neoplasms. Cephalalgia 23:2-5

\section{CONFINIA CEPHALALGICA: 15 YEARS OF ITALIAN PUB-} LISHING ON HEADACHES

${ }^{1,2}$ M. Bartolo, ${ }^{2,3}$ F. Pierelli, ${ }^{1} P$. Rossi, ${ }^{1} S$. Buscone, ${ }^{4} S$. Molinari, ${ }^{I}$ G. Sandrini on behalf of Editorial Board of Confinia Cephalalgica ${ }^{1}$ Headache Unit, and University Centre for Adaptive Disorders and Headache (UCADH), IRCCS Neurological Institute "C. Mondino" Foundation, Pavia, Italy; ${ }^{2}$ University Centre for Adaptive Disorders and Headache (UCADH), Rome, Italy; ${ }^{3}$ Department of Neurology and ORL, University of Rome "La Sapienza", Polo Pontino, ICOT, Latina, Italy; ${ }^{4}$ Direzione Scientifica, IRCCS Neurological Institute "C. Mondino" Foundation, Pavia, Italy; e-mail: michelangelo.bartolo@mondino.it 
Introduction In recent years the quality of medical research in the field of headaches has reached levels of excellence that have amazingly broadened our panorama of knowledge and justified the development in the international publishing field of specialized journals.

Discussion Confinia Cephalalgica was born at the Neurological Institute C. Mondino in Pavia, at the end of the 1980s. At the beginning, it was the official journal of the Italian Society for the Study of Headaches, and then it became the official journal of the University Centres for Adaptive Disorders and Headache. The journal is published 3 times a year and contains original articles, clinical cases of interest, impossible cases, reviews, editorials, therapeutic and historical notes, book reviews and congress reports. The goal of the journal is to constitute a forum for integrating different ideas and expertise through an open dialogue among experts from different disciplines in order to contribute to the advancement and development of knowledge in the field of headaches. The editorial board has always supported the role of the journal as a tool for communication and cultural exchange on headache themes, among Italian-English communities in the world and between Italy and others countries with neolatin language and culture. Not less important is its role as the expression of the patient associations (AL.CE. Group - CIRNA Foundation) that financially supported the journal. During the last ten years (1997-2006), the journal published upon invitation or by direct submission more than 120 papers, furnished by 354 authors. The journal publishes integrated scientific information, promoting a multidisciplinary environment that involves first of all neurologists, but also philosophers and psychiatrists, physiologists and men of literature. After the restyling of the journal cover in 2004, beginning this year, Confinia Cephalalgica is editing, except for special events, only in an online version (free download at the website www.cefalea.it).

Conclusions Although the paper version will be missed, the new electronic online version is looked at as a challenge, with the desire to be of valuable assistance for physicians and researchers. Thus, the future challenge will be the confrontation and exchange of headache information on the Web.

\section{HEADACHES: THERAPEUTIC ASPECTS}

\section{TOPIRAMATE IN MIGRAINE PROPHYLAXIS: AN OPEN STUDY}

R. Iannacchero, F. Peltrone, U. Cannistrà, E. De Caro

Regional Headache Centre, "Pugliese-Ciaccio" Hospital, Catanzaro, Italy; e-mail: rosarioiann@tiscali.it

Introduction Patients who suffer frequent severe migraine or chronic daily headaches are often prescribed prophylactic drugs. A number of beta-blockers, calcium-channel blockers, serotonin agonists, and antidepressants have been tested as prophylactic agents. Topiramate is a new antiepileptic agent that has GABA agonist properties and has few side effects when used in low doses.

Objective and methods The aim of this study was to examine the effects of topiramate on the frequency and intensity of migraine attacks during prolonged use. Patients who demonstrated 3 to 10 migraine attacks per month; in addition to migraine, also had chronic daily headaches from a variety of causes, and all patients had failed treatment with a number of prophylactic agents.

Results We present 35 patients, who kept a daily diary that recorded the frequency, severity of headaches, and the amount and type of rescue medication used. Over a 3-9 months follow-up, 11 patients (30\%) had a very significant improvement in headache frequency (headaches decreased by over $60 \%$ ), and 11 patients had a moderate improvement in headaches (decrease of $40 \%-60 \%$ ). Three patients discontinued therapy due to side effects (lightheadedness, tinnitus, and numbness in hand) and eight patients did not report any improvement despite having been on therapy for over 12 weeks. The remainder of patients had only 8 weeks of therapy.
Conclusions Our open uncontrolled study in patients with resistant headaches suggests that topiramate may be a useful prophylactic medication with limited side effects. A large placebo-controlled study is needed, however, before the results are generalized.

\section{PROPHYLAXIS OF HEMICRANIA CONTINUA WITH TOPI- RAMATE: REPORT OF 2 CASES}

${ }^{I} A$. Palermo, ${ }^{l}$ G. Giglia, ${ }^{2}$ A. Sandullo, ${ }^{l}$ G. Cosentino, ${ }^{l}$ F. Brighina, ${ }^{1}$ B. Fierro

${ }^{I}$ Department of Neurology, Ophthalmology, Otorhinolaryngology and Psychiatry, University of Palermo, Palermo, Italy; ${ }^{2}$ General Medicine Clinic, Sciacca, Agrigento, Italy; e-mail: fbrighina@unipa.it

Introduction Hemicrania Continua (HC) is an uncommon and underrecognized primary headache disorder. It is characterized by a strictly unilateral continuous headache of moderate intensity with possible exacerbations, and it is associated with ipsilateral autonomic features, such as ptosis, miosis, conjunctival injection, lacrimation or rhinorrhoea, that tend to be less pronounced than those seen with cluster headache [1]. The pathophysiology of $\mathrm{HC}$ is poorly understood.

$\mathrm{HC}$ has generally a prompt and enduring response to indomethacin $25-300 \mathrm{mg}$ daily, although $25 \%-50 \%$ of treated patients develop gastrointestinal side effects and unresponsive forms have been described. Patients who cannot tolerate indomethacin pose a difficult management challenge as no other drug is consistently effective in HC. Recently, Matharu et al. [2] reported 2 patients affected by atypical forms of $\mathrm{HC}$ that showed good response to topiramate.

Case reports Here we describe two patients who fulfil both the IHS diagnostic criteria for HC. The physical and neurological examinations and brain magnetic resonance imaging with gadolinium were normal in both patients.

The patients demonstrated a very good response to indomethacin but both developed the most common side effect, gastritis, that led us to stop this treatment. Thus, these patients were started on topiramate with slow titration to $100 \mathrm{mg}$ b.d, and they had a complete benefit with the prophylatic treatment with topiramate after 30 days. The dose of topiramate required to give complete relief of pain was somewhat less than that usually needed for the antiepileptic effect of the drug. There were no side effects during the treatment with topiramate.

Discussion and conclusions Topiramate has multiple mechanisms of action, which include: blockade of the voltage-mediated sodium channels, enhancement of GABA-mediated chloride influx to GABA antagonism of the glutamate kainate/AMPA receptor and carbonic anhydrase inhibition. The mechanism(s) of action responsible for the beneficial effect in various headache syndromes is currently unknown. Whatever the mechanism, if the favourable response observed in our patients can be confirmed in other cases, it would broaden the therapeutic armamentarium available for the treatment of this severe and sometimes incapacitating condition.

\section{References}

1. Headache Classification Subcommittee of The International Headache Society (2004) The International Classification of Headache Disorders, 2nd edn. Cephalalgia 24[Suppl 1]:9-160

2. Matharu MS, Bradbury P, Swash M (2006) Hemicrania continua: side alternation and response to topiramate. Cephalalgia 26:341-344

\section{EFFICACY AND TOLERABILITY OF LEVETIRACETAM IN EPISODIC AND CHRONIC MIGRAINE}

\section{${ }^{I}$ G. Dalla Volta, ${ }^{I}$ S. Griffini, ${ }^{1}$ P. Zavarise, ${ }^{2}$ A. Pezzini}

${ }^{I}$ Neurology Unit, Clinical Intitute, Brescia, Italy; ${ }^{2}$ Stroke Unit, Vascular Neurology, Civilian Hospital, Brescia, Italy; e-mail: dalla@numerica.it

Background Because of its effect in stabilizing neuronal hyperexcitability, the antiepileptic drug levetiracetam is a promising molecule for the prophylaxis of migraine. 
Methods Twenty-five patients with episodic migraine without aura (at least 4 attacks per month of moderate-severe intensity; Group 1) and 18 patients with chronic migraine (no drug abuse; Group 2) were treated with levetiracetam $500 \mathrm{mg} /$ day for 5 days, increased to $1000 \mathrm{mg} /$ day in two administrations, after 5 days. Number of days with headache/month, accompanying symptoms (mild-moderate vs severe), use of symptomatic drugs for migraine attacks (number of drugs), and side effects of levetiracetam were recorded at baseline and at the end of the 3-month treatment period in both groups.

Results One patient in Group 1 did not complete the study because of intolerable side effects (somnolence and asthenia). Patients in Group 1 reported a mean reduction in the number of days/month with headache from 8.4 at baseline to 4.1. In Group 2, 12 patients were nolonger classified as chronic (from 23.8 to 10.3 days/month, mean values), four patients had an improvement of their chronic headache (from 23.8 to 16.2 days/month, mean values) and two had no improvement at the end of the treatment period. Severe accompanying symptoms became mildmoderate in $83 \%$ of patients in Group 1 and in $62 \%$ of patients in Group 2. Drug intake for acute attacks per month changed from 12.3 at baseline to 5.2 drugs in Group 1 and from 32 to 13 drugs in Group 2. Side effects were somnolence (6 patients - 5 patients), asthenia ( 4 and 3 ), postural instability ( 2 and 0 ) in Groups 1 and 2, respectively.

Conclusions This study confirms the role of levetiracetam as a potential preventive treatment for migraine.

\section{COMBINED NEUROMUSCULAR RELAXING AND SHIATSU TREATMENT INTEGRATED WITH MELATONIN IN TEN- SION-TYPE HEADACHE \\ A. Lera, M. Bernardini \\ Headache Centre DSM Giulianova, ASL Teramo, Teramo, Italy; \\ e-mail: antonio.lera@aslteramo.it}

Objective The aim of this study was to evaluate a combined neuromuscular relaxing and Shiatsu programme integrated with melatonin in the treatment of tension-type headache. We tried to consider a possible positive role of physical activity in reducing the frequency and intensity of headache attacks and, in particular, in decreasing activity limitations at a working, social and family level, and therefore, to evaluate the overall improvement in the patients' quality of life.

Patients and methods Before enrolment in the study, 20 middle-aged female subjects underwent psychodiagnostic evaluation with the following scales: MIDAS, MSQOL, MQoLQ, HIT, and MSQ. We utilised the MIDAS scale in this study because it is an objective measure of the severity of a headache attack; it specifies the level of inability and is suitable for identifying the appropriate therapy on the basis of the severity level of each patient. MIDAS scores for our patients were between 6 and 10, which indicated no need for pharmacological therapy, excluding headaches with mild (level 1) and severe inability (levels 3-4).

The study group, consisting of 10 females aged $35-60$ years, underwent combined neuromuscular, relaxing and shiatsu treatment every week, integrated with melatonin retard formulation, 1 tablet per day, for the entire length of the study. For the combined treatment there was a successive prolongation phase: at first, every 15 days for an additional three months, then every month for a further three months. A control group of the same number of subjects did not carry out any treatment programme. At the end of nine months, both the study and control groups underwent another psychodiagnostic evaluation.

Results Comparison of the MIDAS scores between the first and last evaluations showed a decrease of six points in $10 \%$ of cases, five points in $20 \%$, four points in $15 \%$, two points in $25 \%$, with no change in $30 \%$ of cases in the study group. No statistical difference was observed in the comparison of the same group of MIDAS scores in the control group, indicating a positive result only for the combined neuromuscular relaxing and Shiatsu programme integrated with melatonin.

Conclusions On the basis of our results, we can assert that in the study group, which underwent combined neuromuscular relaxing and Shiatsu treatment integrated with Melatonin retard formulation, the quality of life as evaluated by the MIDAS scale was improved significantly. This combination programme seems to have a positive role in reducing the frequency of headache attacks and in alleviating their severity.

\section{EFFECTS OF DENTAL MALOCCLUSION CORRECTION ON} PAIN FROM CHRONIC TENSION-TYPE HEADACHE

L. Di Ianni, E. Tafuri, A. Fabrizio, A. Savini, G. Sidonio, G. Affaitati, R. Lerza, P. de Bigontina, A. Mezzetti, M.A. Giamberardino Headache Centre, "G. D'Annunzio" University of Chieti, Chieti, Italy; e-mail:ldiianni@interfree.it

Introduction Dental malocclusion is a very frequent finding in patients with chronic tension-type headache. The aim of this study was to investigate how malocclusion contributes to both the subjective (number and intensity of attacks) and objective (pain thresholds in muscles of the cervical and upper shoulder regions) painful symptomatology of this type of headache.

Methods Twenty-five patients ( 18 women and 7 men, aged $18-36$ years) were studied. They had been suffering from chronic tension-type headache for at least 1 year prior to examination (diagnosis performed according to IHS criteria) and also presented dental malocclusion. Their headache pain was located in the occipital, parietotemporal and, in 11 cases, also fronto-orbital regions, bilaterally. In all of them pain thresholds to electrical stimulation were measured bilaterally in the trapezius, sternocleidomastoid and splenius muscles before and 30 days after application of the bite to correct dental malocclusion. Measurements were performed in the pain-free interval. Number and maximal intensity (VAS scale) of headache attacks were also recorded in the month of treatment and compared with those relative to the 30 days preceding treatment.

Results Before treatment, pain thresholds in all muscles were significantly lower when compared to thresholds recorded in corresponding sites of 20 non-headache sufferers $(p<0.001)$ (hyperalgesia). In the month of application of the bite there was a significant reduction in the number of headache attacks $(p<0.003)$ but not of their peak intensity; pain thresholds on day 30 were still lower than normal $(p<0.05)$ but significantly increased with respect to pre-treatment values $(p<0.008$ for trapezius, $p<0.004$ for sternocleidomastoid, $p<0.007$ for splenius). A significant linear correlation was found between the decrease in headache pain and the increase in threshold $(p<0.01)$.

Conclusions In patients with chronic tension-type headache, dental malocclusion contributes directly to the triggering of the painful attacks, probably through sensitization of muscles of the cervical/upper shoulder regions.

EFFICACY OF A WORKPLACE COGNITIVE AND PHYSICAL PROGRAMME ON PSYCHOSOMATIC SYMPTOMS IN A WORKING COMMUNITY WITH HEADACHE, NECK AND SHOULDER PAIN: A LONGITUDINAL CONTROLLED STUDY

${ }^{I}$ F. Mongini, ${ }^{I}$ E. Rota,${ }^{2} G$. Ciccone,${ }^{2}$ C. Galassi, ${ }^{1}$ L. Ferrero, ${ }^{1}$ A. Ugolini ${ }^{1}$ Headache-Facial Pain Section, Department of Clinical Pathophysiology, University of Turin, Turin, Italy; ${ }^{2}$ Unit of Cancer Epidemiology, San Giovanni Battista Hospital, Turin, Italy; e-mail: franco.mongini@unito.it

Objective Our purpose was to examine the effect of a workplace cognitive programme on psychosomatic symptoms in a working community. Materials and methods Three hundred and eighty-four employees of the city of Turin, whose working activities involved public contact (registry and tax office), were divided into two groups: study group $(n=192)$ and controls $(n=192)$. In all subjects data regarding headache, neck and shoulder pain, if present, were collected in a standardized fashion. Each subject underwent a physical examination and muscle palpation of pericranial and cervical muscles. The presence of 28 symptoms, mainly psychosomatic in nature, (such as colitis, gastritis, swallowing difficul- 
ties, digestive difficulties, phobias, sleep disorders, palpitations, dizziness, cramps, etc.) were also assessed. These items that belong to those generally investigated when taking a medical history showed in previous studies a significantly higher prevalence in pain patients in respect to a normal population. An instruction programme was then administered to the study group: it consisted of brief shoulder and neck exercises, a relaxation exercise and instructions on how to reduce hyperfunction of the craniofacial and cervical muscles during the day. After six months, all subjects were re-examined in the same way as at baseline and the presence of psychosomatic symptoms was assessed again. In the two groups, the difference between the data at baseline and those at the end was calculated and the data compared (Student's test).

Results In the study group, the mean amount of psychosomatic symptoms decreased significantly $(p=0.002)$ from baseline $(7.27 \pm 5.19 \mathrm{SD})$ to the end $(5.73 \pm 4.77 \mathrm{SD})$. In the control group no significant difference was observed (baseline: $6.65 \pm 5.07 \mathrm{SD}$; end: $6.55 \pm 4.93 \mathrm{SD} ; p=0.78$ ).

Discussion The data demonstrate that the administration of a simple cognitive instruction programme can significantly decrease psychosomatic symptoms in a working community. This finding, in contrast, may be the direct consequence of the cognitive programme, or may be partially due to the beneficial effects of such programme on the headache, neck and shoulder pain in the study population.

Conclusions A cognitive instruction programme can significantly reduce the presence of psychosomatic symptoms in extensive population samples.

\section{EFFECTIVENESS OF AN INTEGRATED APPROACH IN THE PROPHYLACTIC TREATMENT OF CHRONIC TENSION- TYPE HEADACHE}

${ }_{1,2,3}$ B. Ciccone, ${ }^{1,2,3}$ G. Griso, ${ }^{1,2,3}$ S. Lenzuolo

${ }^{1}$ Headache Clinic, Medicine of the Future, Acerra, Naples, Italy; ${ }^{2}$ Medical Diagnostics Clinic, Mercogliano, Avellino, Italy; ${ }^{3}$ Athena Clinic, Saviano, Naples, Italy; e-mail: b.ciccone@libero.it

Introduction Retrospective study of patients from our clinics, suffering from chronic tension-type headache (CTTH), diagnosed in accordance with the criteria of the ICHD-II classification, code 2.3 (OMS G44.2).

Objective The purpose of this study was to demonstrate the effectiveness of an integrated pharmacological and non pharmacological approach compared with an approach that was exclusively pharmacological. Materials and methods We selected 14 patients suffering from CTTH, $14 \mathrm{~F}$, mean age 33 years (range 15-59) \pm 14.1 years, who used an integrated prophylactic therapy, compared with 14 patients suffering from CTTC, $1 \mathrm{M}$ and $13 \mathrm{~F}$, mean age 33 years (range 13-50) \pm 11.7 years, treated solely with medication. The study was carried out over a period lasting from 60 to 180 days. The patients using the integrated approach received daily medication in addition to a weekly session of relational systemic psychotherapy consisting of individual and familial meetings (7 patients) or osteopathic treatment consisting of craniosacral therapy and visceral manipulation (7 patients). The patients in the control group (14) underwent only daily pharmacological prophylactic treatment.

The clinical diary regarding the headache of each patient was reviewed to determine the number of crises per month and to monitor the percentage decrease of the crises.

Results The patient group following the pharmacological prophylaxis had only an average decrease of $64 \%$ in the number of headaches per month.

The patient group using the integrated approach with weekly psychotherapy had an average decrease of $88 \%$, while the patients who underwent craniosacral/visceral manipulation treatment had an average decrease of $92 \%$. Comparison of the two treatments indicated a statistically significant decrease $(p<0.005)$.

Discussion and conclusions The integrated approach in patients with CTTH is more effective than the pharmacological approach alone, as it affects treatment motivation, therapeutic reliability, compliance and the consequent improvement of quality of life.

\section{RATIONAL EMOTIVE BEHAVIOUR THERAPHY (REBT) IN} TENSION HEADACHE

${ }^{I}$ A. Mascaro, ${ }^{I}$ I. Salvaggio, ${ }^{I}$ E. Adducci, ${ }^{I} S$. Barbi, ${ }^{l}$ L. Zappia, ${ }^{2}$ G. Di Trapani

${ }^{1}$ Department of Anaesthesiology and Intensive Care and ${ }^{2}$ Department of Neurology, Catholic University Medical School, Rome, Italy; e-mail:iclar@rm.unicatt.it

Introduction During the last few years, many studies have demonstrated how an emotional condition can influence pain. This aspect is especially evident for headache, in which the connection between emotionality, pain, intensity and frequency is very important. In the Centre for Pathophysiology and Pain Therapy of the Catholic University Medical School of Rome, we studied a Rational Emotive Behaviour Therapy (REBT) that underscores the importance of patients' cognitive processes in the genesis and feeding of emotive and behavioural disturbances. Materials and methods From September 2004 to April 2006, after informed consent, we studied 60 patients ( 35 females and 25 males, mean age 35 years). Their tension headaches (ICHD-II criteria, 2004) were bilateral with occipital localization, without aura, vomiting, photophobia and phonophobia; they had eight crises per month and visual analogue scale (VAS) score $=7$.

Their clinical history, objective and neurological examinations excluded a secondary headache. We had an indepth conversation with each patient, and we noticed that everyone was depressed or anxious or hostile; we gave them some forms to fill out and a headache diary to complete before and after the start of therapy. They were divided into two randomised groups of 30 patients. The first group was treated with cognitive, emotional and behavioural techniques; the second group was treated with pharmacological therapy with amitriptyline $30 \mathrm{mg} /$ day, alprazolam $0.25 \mathrm{mg} /$ day and celecoxib $200 \mathrm{mg}$, if needed. They were treated for 6 months.

Results In the first group, treated with cognitive psychotherapy, VAS decreased from 7 to 3 , the crises decreased from 8 to 2 , and the emotional condition improved with a reduction of depression, anxiety and hostility. In the second group, treated with pharmacological therapy, VAS decreased from 7 to 2, and the crises decreased from 8 to 3 .

Conclusions From our results we observed that patients treated with cognitive psychotherapy showed improvement in VAS scores and their emotional condition, and reduction in the number of montly crises, while patients treated with pharmacological therapy also had improved VAS scores and reduction in the number of crises, but with no modifications of their emotional condition.

\section{GYMNASTIC-PHYSICAL ACTIVITY: PERFECT SHAPE FUNCTION IN MIGRAINE AND CLUSTER HEADACHE}

${ }^{1}$ A. Lera, ${ }^{1}$ M. Bernardini, ${ }^{2}$ C. Germani

${ }^{1}$ Headache Centre DSM Giulianova, ASL Teramo, Teramo, Italy; ${ }^{2}$ Institute of Experimental Research for Motor Sciences Life Quality Project, S. Benedetto del Tronto, Ascoli Piceno, Italy; e-mail: antonio.lera@aslteramo.it

Objective The aim of this study was to evaluate the effects of a programme of gymnastic-physical activity (Perfect Shape System) on the quality of life in a group of subjects suffering from migraine and cluster headache. We tried to consider a possible positive role of physical activity in reducing the frequency and intensity of headache attacks and, in particular, in decreasing inability at a working, social and family level, and therefore, improving overall the patients' quality of life. Patients and methods Before enrolment in the study, forty subjects suffering from primary headaches ( 30 with migraine and 10 with cluster headache) underwent clinical evaluation with the following scales: MIDAS, MSQOL, MQoLQ, HIT, and MSQ. We utilised the MIDAS scale in this study since it is an instrument which objectively measures the severity of a headache attack; it specifies the inability level and is suitable for identifying the appropriate therapy on the basis of the 
severity level of each patient. MIDAS scores for our patient group were between 6 and 10, which indicated no need for pharmacological antimigraine therapy, excluding headaches causing mild (level 1) and severe inability (levels 3-4).

The study group consisted of 20 females aged 35-60 years, who underwent gymnastic-physical activity through the methodical Perfect Shape System, which lasted two months, followed by further activities for an additional six months. A control group of equal number of subjects did not carry out any gymnastic-physical programme at all. At the end of eight months, all 40 patients of both the study and control groups, underwent another clinical evaluation.

Results Comparison of the MIDAS scores between the first and last evaluations showed a decrease of seven points in $5 \%$ of cases, six points in $5 \%$, five points in $15 \%$, three points in $10 \%$, one point in $20 \%$, with no change in $45 \%$ of cases for the subjects who practised Perfect Shape. No statistical difference was observed in the comparison of the same group of MIDAS scores in the control group, indicating a positive result only for the patients who followed the Perfect Shape programme.

Conclusions On the basis of our results, we can assert that in the study group, which followed the Perfect Shape System, the quality of life as evaluated by the MIDAS scale improved, although in a different way, with reduction in negative social, working and family effects. This is in marked contrast to the control group, who showed no improvement in quality of life parameters. Gymnastic-physical activity and, in particular, the Perfect Shape programme, seem to contribute in a positive way to reducing the frequency of headache attacks and to alleviating their severity. However, there were no relevant changes in terms of quality of life parameters between the different forms of primary headaches.

\section{USE OF COMPLEMENTARY AND ALTERNATIVE MEDICINE BY PATIENTS WITH CLUSTER HEADACHE: RESULTS OF A MULTI-CENTRE HEADACHE CLINIC SURVEY}

${ }^{1,2}$ P. Rossi, ${ }^{3}$ P. Torelli, ${ }^{1}$ C. Di Lorenzo, ${ }^{2}$ G. Sances, ${ }^{3}$ G.C. Manzoni, ${ }^{2}$ C. Tassorelli, ${ }^{2,4}$ G. Nappi

${ }^{1}$ Headache Clinic, INI Grottaferrata, Grottaferrata, Rome, Italy; ${ }^{2}$ UCADH Pavia, Italy; ${ }^{3}$ UCADH Parma, Italy; ${ }^{4}$ University of Rome "La Sapienza", Rome, Italy; e-mail: paolo.rossi90@alice.it

The use of complementary and alternative medicine (CAM) in cluster headache $(\mathrm{CH})$ is a phenomenon about which little is known. This study was undertaken to evaluate the rates, pattern, satisfaction and presence of predictors of CAM use in a clinical population of patients with $\mathrm{CH}$. One hundred $\mathrm{CH}$ patients attending three headache clinics were asked to undergo a physician-administered structured interview designed to gather information on CAM use.

Past use of CAM therapies was reported by $29 \%$ of the patients surveyed, with $10 \%$ having used CAM in the previous year. Only $8 \%$ of the therapies used were perceived as effective, while a partial effectiveness was reported in $28 \%$ of CAM treatments. The most common source of recommendation of CAM was a friend or relative $(54 \%)$. Approximately $62 \%$ of CAM users had not informed their medical doctors of their CAM use. The most common reason for deciding to try a CAM therapy was that it offered a "potential improvement of headache" $(44.8 \%)$. Univariate analysis showed that CAM users had a higher income, had a higher lifetime number of conventional medical doctor visits, had consulted more headache specialists, had a higher number of $\mathrm{CH}$ attacks per year with disease and had a significantly higher proportion of chronic $\mathrm{CH}$ vs episodic $\mathrm{CH}$. A binary logistic regression analysis was performed and two variables remained as significant predictors of CAM use, the income level (OR=5.7, 95\% $\mathrm{CI}=1.6-9.1, p=0.01$ ), and the number of attacks per year with disease $(\mathrm{OR}=3.08,95 \% \mathrm{CI}=1.64-6.7, p<0.0001)$.

Our findings suggest that $\mathrm{CH}$ patients, in their need of and quest for care, seek and explore both conventional and CAM approaches, even if only a very small minority finds them satisfactory. Conventional MDs and CAM practitioners should be made aware of this redefinition of the role of modern biomedicine, given that they are in a unique position to provide patients with help and guidance.

\section{STANDARDIZED APPROACH TO PATIENTS WITH MIGRAINE IN A HEART-BRAIN CENTRE: 6-MONTH EXPERIENCE}

${ }^{I} G$. Dalla Volta, ${ }^{1} B$. Troianiello, ${ }^{I} S$. Griffini, ${ }^{1} P$. Zavarise, ${ }^{2} A$. Pezzini, ${ }^{3}$ A.M. Lanzone, ${ }^{3}$ R. Albero, ${ }^{4}$ M. Guindani

${ }^{1}$ Neurology Unit, Clinical Intitute, Brescia, Italy; ${ }^{2}$ Stroke Unit, Vascular Neurology, Civilian Hospital, Brescia, Italy; ${ }^{3}$ St. Rocco Cardiology Clinic, Brescia, Italy; ${ }^{4}$ Clinical Institute S. Anna, Brescia, Italy; e-mail: dalla@numerica.it

Background Because a pathogenic relationship between patent foramen ovale (PFO) and migraine with aura is assumed, percutaneous closure of PFO has been recently proposed as an effective treatment. However, definitive criteria to identify a priori migraine patients who will benefit from PFO closure and those whose migraine will not be influenced by this treatment are lacking. We propose the standardized approach to these patients in use at the Heart-Brain Centre of the Clinica St Rocco of Ome, Brescia.

Methods During a 6-month period, 120 patients with migraine with aura, 48 patients with migraine without aura, and 18 patients with cluster headache were evaluated. All subjects underwent a contrastenhanced transcranial Doppler (TCD) investigation to detect the presence/absence of PFO. This was diagnosed in $62.5 \%$ of cases with aura, $37.5 \%$ of those without aura, and $38.8 \%$ of cluster headache. In the case of a positive finding, a standardized work-up including brain MRI, thrombophilic tests (proteins $\mathrm{C}$ and $\mathrm{S}$, antithrombin III, APCR, antiphospholipid antibodies, total plasma homocysteine, vitamin B12 and folate levels, G1691A polymorphism of factor V, G20210A polymorphism of factor II, C677T polymorphism of MTHFR), ultrasound evaluation of the deep veins of the legs and the carotid-vertebral arteries was carried out. Transesophageal echocardiography was performed in cases of PFO-positivity with curtain effect, MRI evidence of ischemic lesions (MRI+), or evidence of a prothrombotic state $(\mathrm{T}+)$. This allowed the identification of co-existent atrial septal aneurysm (ASA).

Discussion and conlusions Based on these findings, $\mathrm{PFO}$ closure can be considered in cases with co-existent ASA when at least one of the following conditions is present: curtain pattern on TCD, MRI+, or T+. It can also be considered in those cases without co-existent ASA in which MRI+ or T+ are present.

Although the management of PFO in patients with migraine is still a matter of debate, the proposed standardized approach can be of help in selecting patients who will benefit from percutaneous closure in clinical practice.

\section{EPIDURAL BLOOD PATCH IN THE TRENDELENBURG POSI-} TION TO TREAT HEADACHE BY SPONTANEOUS CSF LEAK

${ }^{1}$ E. Ferrante, ${ }^{2}$ I. Arpino, ${ }^{1}$ A. Citterio, ${ }^{2} R$. Wetzl, ${ }^{2}$ A. Savino, ${ }^{1} R$. Sterzi ${ }^{I}$ Neurosciences and ${ }^{2}$ Emergency Departments, Niguarda Cà Granda Hospital, Milan, Italy; e-mail: enricoferrante@libero.it

Background Spontaneous intracranial hypotension $(\mathrm{SIH})$ generally results from spontaneous spinal CSF leakage [1]. Treatment is usually conservative but epidural blood patch (EBP) has emerged as the most important nonsurgical treatment for SCSFL [2].

Objective To evaluate the efficacy of epidural blood patch (EBP) in the Trendelenburg position in the treatment of headache by spontaneous CSF leakage (SCSFL).

Materials and methods We observed 30 patients with SCSFL between 1992 and 2005. Eleven patients (6 women and 5 men; age range 31-66 years, mean age 40) received EBP. All patches were performed in the lumbar region, using 15 to $30 \mathrm{~mL}$ (mean $23 \mathrm{~mL}$ ) of autol- 
ogous blood. All patients mantained a $30^{\circ}$ Trendelenburg position during the procedure and for 24 hours after the procedure. Follow-up ranged from 6 months to 2 years.

Results All patients had orthostatic headaches. Other manifestations were nausea, vomiting, mild neck stiffness, tinnitus, blurred vision, diplopia and bilateral upper limb numbness. CT myelography, spinal MRI or brain MRI or radionuclide cisternography showed CSF leakage sites in 6 out of 11 patients; 2 sites were at the cervical level, the others at the lumbar level. In 10 out of 11 patients, brain MRI showed diffuse pachymeningeal gadolinium enhancement (neuroimaging of intracranial hypotension). All patients failed an initial conservative treatment, which consisted of bed rest and hydration, over a period of 1 to 13 months. All treated patients became asymptomatic, 1 responded only after $3 \mathrm{EBP}$, and 1 had a residual mild headache during the Valsalva manoeuvre for 2 months. Until now, none has had a relapse.

Discussion and conclusions Our data confirm the efficacy of EBP in the SCSFL headache and suggest also the importance of a prolonged Trendelenburg position especially when the leak site is at the cervical level and the autologous blood patch in the lumbar region is performed. References

1. Ferrante E, Savino A, Sances G, Nappi G (2004) Spontaneous intracranial hypotension syndrome: report of twelve cases. Headache 44:615-622

2. Turnbull DK, Shepherd DB (2003) Post-dural puncture headache: pathogenesis, prevention and treatment. $\mathrm{Br} \mathrm{J}$ of Anaesth 91:718-729

\section{HEADACHE IN CHILDHOOD AND ADOLESCENCE I}

\section{CLIMATIC VARIATIONS AND MIGRAINE IN CHILDHOOD}

D. Moscato, M.I. Peracchi

Headache Centre, Saint Charles Hospital, Rome, Italy; e-mail: dmoscat@tin.it

The incidence of migraine in childhood has increased in recent years. Up to now that increase has been attributed to the worsening of childern's conditions: the job of both parents, the long distance to school, the high number of separations, divorces etc. [1, 2].

Regardless, the environmental situation, unexpected climatic variations, and elevated levels of pollutants, may also have had a specific influence on migraine.

We tried to estimate the influence of these factors in a group of 120 children (72 F, $48 \mathrm{M}$, age range 5-17 years) affected by migraine without aura $(n=112)$ and migraine with aura $(n=8)$, assessed from 1995 to 2005 . For these patients the headache triggers were evaluated (Table 1).

No significant variations were found in the percentage of stress as a trigger factor, whereas the number and percentages of patients referring atmospheric variations and solar exposure as a triggering factor increased over time.

Table 1 Trigger Factors

\begin{tabular}{lrrrrrrr}
\hline & 1995 & 1997 & 1999 & 2001 & 2003 & 2005 & Total \\
\hline Emotional stress, \% & 90 & 90 & 80 & 90 & 100 & 70 & 83 \\
Physical stress, \% & 50 & 30 & 30 & 40 & 60 & 30 & 40 \\
Environment stress, \% & 70 & 80 & 90 & 50 & 90 & 60 & 73 \\
Lights, \% & 50 & 40 & 60 & 30 & 70 & 40 & 48 \\
Smell, \% & 40 & 20 & 40 & 60 & 30 & 40 & 33 \\
Smoke, \% & 20 & 40 & 20 & 40 & 10 & 20 & 25 \\
Food, \% & 20 & 20 & 20 & 10 & 00 & 10 & 15 \\
Climatic Variations, \% & 40 & 50 & 60 & 60 & 70 & 80 & 60 \\
Solar exposure, \% & 10 & 20 & 40 & 60 & 90 & 70 & 48 \\
\hline
\end{tabular}

These preliminary data need to be confirmed by further studies. Among triggers examined, solar exposure as a controllable trigger should be avoided or limited to reduce its influence in inducing migraine attacks.

References

1. Moscato D, Scurci M (1987) Headache in childhood. epidemiological study. Cephalalgia 7[Suppl 8]:359-360

2. Moscato D, Falasconi A, Bochicchio F (2003) New characteristics of children's headache. Cephalalgia 23:602

\section{TENSION-TYPE HEADACHE IN CHILDREN}

\section{A. Forabosco, T. Grandi, M. Malavasi}

University of Modena and Reggio Emilia, School of Dentistry, Modena, Italy; e-mail: forabosco.andrea@unimo.it

Introduction Tension-type headache is a common problem among children in primary school. Dental malocclusion can often be the cause of this form of primary headache [1].

Materials and methods Twenty schoolchildren who had already received a diagnosis of primary headache treated with analgesic substances came to our clinic. An orthodontic evaluation and orthopanoramic X-ray film were performed. All patients were found suffering from malocclusion. Limitation of the functional mandible's movements, teeth abrasions, and hypertrophy of the mandibular elevator muscles were present. To treat muscular problems originating from the stomatognathic system, an occlusal splint was performed. Intensity [using a graphic rating scale (GRS)] and frequency (daily, weekly, monthly) of headache before treatment (T0), after one month (T1), after three months (T2) and after six months (T3) were recorded. Wilcoxon test was applied for statistical evaluation.

Results All patients showed a considerable reduction of their symptoms. A significant improvement of the intensity and the frequency of headache attacks was registered after treatment $(p<0.0001)$.

Discussion and conclusions The high occurrence of malocclusion in the subjects with tension-type headache seems to play an important role in the genesis of headache. At present, the treatment for patients who suffer from these disorders has increasingly required the need for a collaborative team approach. An orthodontic treatment should be suggested, both for an improvement of the headache and the functional aspects, but the results may not be significant before 2 years [2]. An application of a plate bite, without any drug therapy, can be the treatment of choice to reduce the symptomatology in children suffering from tension-type headaches.

\section{References}

1. Moscato D, Banaudi E, Zino G (2000) Malocclusion affect the occurrence of child headache: preliminary data. Cephalalgia 20:285

2. Henrikson T (1999) Temporomandibular disorders and mandibular function in relation to Class II malocclusion and orthodontic treatment. A controlled, prospective and longitudinal study. Swed J Dent Suppl 134:1-144

\section{HEADACHE AND QUALITY OF LIFE IN A CHILD AND ADO- LESCENT COHORT}

\section{${ }^{1}$ D. Brovia, ${ }^{2}$ S. Cesi, ${ }^{3}$ M.M. Cainazzo, ${ }^{3}$ L.A. Pini}

${ }^{1}$ School of Specialization in Medical Toxicology, ${ }^{2}$ School of Specialization in Childhood Neuropsychiatry, University of Modena and Reggio Emilia, Modena, Italy; ${ }^{3}$ Toxicology and Clinical Pharmacology, University Hospital of Modena, Modena, Italy; e-mail: pinila@unimore.it

Introduction Up to $10 \%$ of children between the ages of 5 and 15 have migraines [1], while an even higher number of adolescents have migraines [2].

The primary aim of this study was to evaluate the quality of life in young patients, using the PED-MIDAS, the QLH-Y Questionnaire and 
Children's Depression Inventory (CDI) scales. A secondary end-point was to examine the changes in clinical picture and diagnoses related to headache treatment.

Methods and patients The study included 70 patients (32 F, 38M; age range: $8-15$ years, mean age $12.34 \pm 1.91$ years) visited in the Modena Headache Centre between January 2002 and June 2004. At the initial visit, each patient underwent complete history-taking and general and neurological examinations in the presence of a parent. A second follow-up visit was performed in the period January-June 2005; headaches were diagnosed according to the International Headache Society criteria (ICHD-II).

Questionnaire At the follow-up visit, the patients were asked to check MIDAS, QLH-Y and CDI Questionnaires in the presence of a trained doctor. They were urged to express their own judgements, but they could ask a parent for advice.

Results All 70 patients completed the study. Mean illness duration was 44.31 months. Forty-two patients had migraine without aura, 10 had migraine with aura, 18 had tension-type headache (16 with episodic tension-type headache (ETTH) and 2 with chronic tension-type headache (CTTH)). Sixty-eight out of 70 patients confirmed the first diagnosis. The frequency of headache was related to the degree of disability, while the intensity was moderate-severe in almost all patients in spite of disability scores.

Pathological values found in Children's Depression Inventory (CDI) at the follow-up visit showed that almost $50 \%$ of patients had a bad perception of themselves with devaluation and often with feelings of desperation, and thus referred disobedience, asthenia and a negative body image. According to the QLH-Y data, our patients seemed to experience stress, fatigue and somatic symptoms. The most used drug for headache attack was acetaminophen.

Discussion The results of this study suggest that the prevalence of migraine without aura and ETTH decreases with increasing age. Optimal care for children and adolescents with headache can be realized with collaboration among primary care practitioners and headache specialists; behavioural treatment is a foundation for provision of such care. References

1. Abu-Arefeh I, Russell G (1994) Prevalence of headache and migraine in school children. BMJ 309:765-769

2. Split W, Neuman W (1999) Epidemiology of migraine among students from randomly selected secondary schools in Lodz. Headache 39:494-501

\section{HEADACHE AND EPILEPSY IN CHILDREN}

E. Tozzi, R. Savini, I. Florio, A. Di Fonzo, B. Giobbi

Paediatric Clinic, University of L'Aquila, L'Aquila, Italy; e-mail: etozzi@univaq.it

Introduction The association between headaches and epileptic seizures is a well-known phenomenon. The headache could be periictal and interictal, in $50 \%$ of epileptic patients [1]. The incidence of interictal headache is not clear, but many authors have shown that the incidence of interictal migraine headache was significantly higher in patients with migraine-like postictal headache [2]

Materials and methods Forty-three children aged 4-18 years, 22 males and 21 females, affected by epilepsy and suffering from headache, were studied in order to verify the correlation between periictal and interictal headache. Headache was diagnosed according to ICHD-II criteria, and epilepsy according to ILAE 1989 criteria.

Epilepsy was diagnosed as: Benign focal epilepsy with vertex spikes and waves (BVSWE) in 9 children; reflex photosensitive epilepsy in 11 children, occipital epilepsy in 5 children, juvenile myoclonic epilepsy in 4 children, and generalized epilepsy in 12 children.

The headache diagnosis was: migraine without aura (MO) in 18 children, migraine with aura (MA) in 4 children, tension-type headache (TTH) in 4 children, migraine-like and tension-type like headache in 6 children, and stabbing headache in 5 children.
Results In 20 children, the headache started before epilepsy began and in 5 children after epilepsy began. In 26 children, the diseases were contemporary. There was no correlation between the region of EEG anomalies and the side of pain. Only 3 children, suffering from BVSWE, and 5, suffering from generalized epilepsy, showed headache in the vertex region.

In 11 children $(25 \%)$, there was a temporal relationship between headache and epilepsy. In 4 patients with seizures while sleeping, the headache occurred upon awakening, in 3 children with occipital epilepsy the headache was postictal, and in 4 children with photosensitive epilepsy the headache was preictal and was triggered by photic stimuli (TV, play station).

The preictal headache was defined by children as stabbing, the postictal headache as migraine-like.

Conclusions Migrainous-like headache is prevalent among epileptic children during the peri-ictal and interictal periods. In fact, migrainelike headache was found in $50 \%$ of patients with photosensitive epilepsy and migraine in $100 \%$ of children with rolandic epilepsy.

References

1. Bernasconi A (2005) Peri-ictal Headache. Epilepsia 46[Suppl 6]:47

2. Yamane LE, Montenegro MA, Guerreiro MM (2004) Comorbidity

headache and epilepsy in childhood. Neuropediatrics 35:99-102

\section{PREVALENCE OF MIGRAINE IN FAMILIES OF CHILDREN WITH PANAYIOTOPOULOS SYNDROME}

P. Mariotti, P. De Rose, F. Perrino, D. Martinelli, R. Chiera, S. Staccioli, A. Sacco, G. Vasco, D. Lettori, D. Battaglia

Unit of Child Neuropsychiatry, Policlinic "A. Gemelli", Catholic University, Rome, Italy; e-mail: pmariotti@rm.unicatt.it

Introduction Early-onset benign occipital seizure susceptibility syndrome or the "Panayiotopoulos type" of childhood epilepsy with occipital paroxysms (PS) is a partial form of epilepsy that occurs in $13 \%$ of children aged 3-6 years with one or more non-febrile seizures [1]. Although many authors have studied the relationship between headache and PS, there do not seem to be any clear results [1].

Aim The aim of our study was to determine the relationship between migraine and PS in childhood and the prevalence of family history of migraine and epilepsy in patients with PS.

Patients and methods This was a retrospective study conducted at the Child and Adolescent Neuropsychiatry Unit of our University Hospital, in patients recruited between 1999 and 2006, diagnosed as PS on the basis of the ILAE Task Force on Classification and Terminology criteria. Anamnestic, clinical, and instrumental data were collected to clear the semiology and the frequency of the seizures. All patients underwent a video-EEG during wake and sleep cycles with HP and ILS, and a brain MRI. All patients were given the specific record for headache in children and adolescent patients of the Italian Child and Adolescent Neuropsychiatry Society Group. The diagnosis of migraine was based on the criteria of The International Classification of Headache Disorders, 2nd edition (ICHD-II).

Migraine prevalence in children with PS and in their families was compared with a member of the general population of the same age group. Results We evaluated 20 patients with PS, 11 males and 9 females, mean age at the end of follow-up $=8.5$ years, range 4 to 14 years. Among these, $7 / 20(35 \%)$ had migraine (six migraine without aura and one with visual aura). Regarding family history: $13 / 20$ cases $(65 \%)$ were positive for migraine, 5/20 (25\%) for epilepsy, $4 / 20$ (20\%) for both, and $4 / 20$ for febrile seizures.

All children with migraine also had a family history of migraine. The headache usually started in the same year in $2 / 7$ cases $(29 \%)$ or after the diagnosis of epilepsy in $5 / 7$ (71\%).

Conclusions So far, no study has showed a clear relationship between a family history of migraine and PS. In particular, Panayiotopoulos says "there is no family history of similar disorders of benign childhood seizure susceptibility, epilepsies or migraine" in children with PS [2]. 
To our knowledge, this is the first retrospective study to report a prevalence of $65 \%$ for migraine, $25 \%$ for epilepsy, and $20 \%$ for both in family members of affected children. PS and migraine are probably genetically determined. Our study supports the hypothesis of a clinical continuum between migraine and epilepsy, particularly PS that share similar characteristics and a familial spectrum.

References

1. Andermann F, Zifkin B (1998) The benign occipital epilepsies of childhood: an overview of the idiopathic syndromes and of the relationship to migraine. Epilepsia 39[Suppl 4]:S9-S23

2. Panayiotopoulos CP (2005) Benign childhood focal seizures and related epileptic syndromes. In: Panayiotopoulos CP (ed) A clinical guide to epileptic syndromes and their treatment. Oxford: Bladon Medical Publishing, 2002, pp 101

\section{HEADACHE AND CARDIO-EMBOLIC STROKE: THE IMPOR- TANCE OF CLINICAL SUSPICION. REPORT OF A PAEDI- ATRIC CASE}

${ }^{I}$ G. Indolfi, ${ }^{1} S$. Trapani, ${ }^{I}$ C. Massai, ${ }^{2}$ L. Calistri, ${ }^{2}$ C. Scalas, ${ }^{1} M$. Resti ${ }^{I}$ Department of Paediatrics, University of Florence, Anna Meyer Paediatric Hospital, Florence, Italy; ${ }^{2}$ Children's Headache Centre, Paediatric Neurology Clinic, Anna Meyer Paediatric Hospital, Florence, Italy; e-mail: g.indolfi@meyer.it

A 14-year-old boy, with an underlying history of asymptomatic ostium secundum atrial septal defect and a family history negative for vascular diseases or coagulation disorders, was admitted to the emergency department of a general hospital because of a severe headache and visual disturbance that began during a school examination. As brain CT scan and fundoscopic examination were normal, he was dismissed with the diagnosis of migraine with aura and symptomatic therapy. Twelve hours later for the persistence of the same complaints, he was newly admitted: physical examination showed right homonymous hemianopsia and a systolic ejection murmur, but no other abnormalities. EEG showed bioelectric abnormalities on the left temporal and occipital regions and magnetic resonance imaging revealed acute left occipital infarction and normal head and neck vessels. He was then transferred to our tertiary care paediatric hospital; in the following days his clinical conditions were stable. An expanded investigation for an underlying prothrombotic tendency showed increased plasma homocysteine (29 micro$\mathrm{mol} / \mathrm{L}$ ) and homozygosity for the methylenetetrahydrofolate reductase gene $\mathrm{C} 677 \mathrm{C}>\mathrm{T}$ polymorphism. Anticoagulant therapy, folic acid and vitamin B12 supplementation was given and the 6-month follow-up was uneventful: right temporal hemianopsia persisted. Atrial septal defect was subsequently closed via percutaneous approach.

Arterial ischemic stroke is being increasingly diagnosed and recognized in childhood. Recognition of clinical stroke may be difficult, particularly in infants and young children. Ischemic events are usually suspected when a child presents with abrupt onset of a hemiparesis but the initial symptom can be a common complaint, such as isolated headache and/or subtle and difficult to recognize focal sensory deficits or visual disturbances.

A detailed history focused on the most frequent risk factors, such as congenital thrombophilic states, cardiac diseases, infections, trauma and sickle cell anemia, represents the initial screening for the diagnosis of paediatric stroke since multiple risk factors commonly coexist in individual patients [1]. As demonstrated by our case report, magnetic resonance is the imaging modality of choice to confirm that an acute ischemic event has occurred and to determine the extent and location of acute cerebral injury, the patency of major neck and intracranial arteries and relative cerebral perfusion.

It is crucial to increase clinician awareness of stroke also in children as clinical suspicion can reduce the time lag to diagnosis and to appropriate treatment of this disease [2].

\section{References}

1. Lanthier S, Carmant L, David M et al (2000) Stroke in children: the coexistence of multiple risk factors predicts poor outcome. Neurology 54:371-378

2. Gabis LV, Yangala R, Lenn NJ (2002) Time lag to diagnosis of stroke in children. Pediatrics 110:924-928

\section{HEADACHE IN ADOLESCENTS: SYMBOLIC MEANING BETWEEN CLINICAL DATA AND INNER PSYCHIC LIFE} D. Ragusa, D. Puma, V. Raieli

Division of Child Neurology and Psychiatry, "G.F. Ingrassia" Hospital, AUSL 6, Palermo, Italy; e-mail: donatellaragusa@inwind.it

Introduction Primary headaches can present occasional and nonspecific anatomical and/or functional anomalies, but although they do not have an aetiological and pathophysiological role in producing painful attacks, they can influence the symptomatic course through the symbolic meaning that they have for the patients and their family. We present and analyse three cases of adolescents with these characteristics. Case 1: A twelve-year-old boy with a story of migraine-type attacks occurring every weekend for the last three months; brain MRI showed a pineal cyst and for this reason he underwent neurosurgical consultation and another MRI with gadolinium; during the clinical evaluation, migraine appeared the day before every examination and afterward, he was migraine-free for long periods.

Case 2: An eleven-year-old boy suffering from tension-type headache and depressive symptoms: his father was operated on for cerebral aneurysm, the boy's EEG showed positive occipital sharp waves, evoked by all IPS frequencies; undergoing MRI, which was normal, and clinical interviews regarding the family's fears, in the last two years, the boy nolonger suffered from headache.

Case 3: A fourteen-year-old girl presented with very frequent and severe migraine-type attacks for about two months; she had been wearing an orthodontic appliance for over two years. The decision to remove the orthodontic appliance resulted in the disappearance of the headache.

Discussion The nonspecific anomalies, considered as symbols, found in these three clinical cases are "dumb elements" [1], but they represent an important meaning in the patients' inner psychic life and may induce the manifestation of some developmental emotional problems. In the first case, pineal cyst may represent a need of dependence in the relationship between the patient and his busy family and help him to receive attention and be listened to. In the second case, the abnormal EEG and the father's history of cerebral aneurysm produced in the boy an identity problem with a father who was always absent. The clinical and psychological therapy and normal brain MRI examination disintegrated the "symbol" with the disappearance of the headache. In the third case, the relationship with a very perfectionist mother resulted in the onset of migraine-type attacks. The removal of the orthodontic appliance allowed the patient to become independent without being forced to accept a selfimage that the mother imposed.

Conclusions The three cases, with their different data and life events, confirm that nonspecific signs need attention, because their symbolic meaning may be significant and influence the course of pain.

\section{Reference}

1. Freud S (1915-1918) Vorlesungen zur Einfuhrung in die Psychoanalyse. G.W. vol. XI, 151

\section{PRESENT AND PAST PSYCHOPATHOLOGY IN CHILDHOOD MIGRAINE: A STUDY WITH THE CHILD BEHAVIOUR CHECKLIST}

F. Salvadori, V. Gelmi, F. Muratori

Division of Child Neuropsychiatry, Scientific Institute Stella Maris, Department of Neurosciences, University of Pisa, Pisa, Italy; e-mail: francesco.salvadori@inpe.unipi.it 
Introduction A strong association has been observed between migraine and psychiatric problems such as depression and anxiety.

Objective The aim of this study was to describe the premorbid state of migraine in children by using a structured questionnaire, designed to obtain standardized reports of children's behavioural problems and capabilities, as observed by parents.

Subjects and methods Thirty subjects with migraine and 30 healthy subjects were enrolled in a case-control study. Using the Child Behaviour CheckList (CBCL), ratings were obtained for maternal memories of the past for two age periods ( $0-3$ years and $4-6$ years) and for the present state (7-16 years). The CBCL scores of the migraine group (MG) were compared to those of the control group (CG) for the three periods. A longitudinal analysis was performed to evaluate the evolution of psychopathology, by comparing the CBCL scores of the MG in the three age periods.

Results In the 7-16 year group, children of the MG had significantly higher mean CBCL scores - i.e., nearer to the clinical threshold - than those of the CG in several scales: total problems $(p<0.01)$, internalizing $(p<0.001)$, somatic $(p<0.001)$, anxious/depressive $(p<0.01)$, thoughts $(p<0.05)$ and attention problems $(p<0.01)$. At $4-6$ years, significantly higher scores between the MG and the CG were found in the total problems scale $(p<0.001)$, internalizing $(p<0.001)$, somatic $(p<0.001)$, anxious/depressive $(p<0.001)$, attention $(p<0.01)$, social problems $(p<0.01)$ and withdrawn $(p<0.05)$. At $0-3$ years, significantly higher scores between the MG and the CG were found in the total problems scale $(p<0.01)$, internalizing $(p<0.05)$, somatic $(p<0.001)$, and anxious/depressive $(p<0.01)$. In a longitudinal perspective we did not demonstrate statistically significant differences between 7-16 and 4-6 year age periods. Comparing the age periods of $4-6$ years and $0-3$ years, significant differences were found in total problems $(p<0.01)$, internalizing $(p<0.001)$, somatic $(p<0.01)$ and also in externalizing $(p<0.01)$ and aggressive syndrome scale $(p<0.01)$, but not in the anxious/depressive scale.

Discussion Our study suggests the association between migraine, depression and anxiety during childhood and adolescence. The internalizing component (depression and anxiety) may represent a persistent factor that feeds the migraine. The demonstration of an internalizing score in a headache patient, by means of the CBCL, could be of the utmost importance to prevent migraine from becoming chronic.

Conclusions Migraine may begin during childhood/adolescence with a symptomatology, which may have its roots in previous behavioural problems. Even if they rarely reach the clinical threshold with the CBCL questionnaire, children who develop migraine are different from healthy children for the higher rate of behavioural problems. Migraine could be considered the expression of a previous vulnerability.

\section{HEADACHE: WHAT DO CHILD AND MOTHER WANT FROM THEIR PHYSICIAN?}

V. Raieli, G. La Franca, M. La Vecchia, E. Pandolfi, D. Puma, D. Ragusa, R. Ceraulo, M. Eliseo

Division of Child Neuropsychiatry "L. Biondo", Ingrassia Hospital, A.U.S.L.6, Palermo, Italy; e-mail: vinzi.raielk@inwind.it

Introduction To establish a good "strategic compliance" between the doctor, and the young headache patient and the patient's family, it is important to know what the mothers or the children want from paediatricians and neurologists.

Objective The aim of this study was focused on child's and mother's expectations regarding the diagnostic and therapeutic approaches from the paediatrician and neuropsychiatrist. Moreover, we wanted to know the doctors' opinions.

Methods We selected 100 consecutive children older than 10 years with primary headaches, admitted for the first time to our department. A questionnaire was administered individually to the children and to all 100 mothers before the visit. It had several multiple choice questions about the child's and mother's fears, their expectations and opinions regarding medical visits, and symptomatic and prophylactic therapy. A short questionnaire was also administered to 50 paediatricians, having common characteristics previously selected with a questionnaire.

Results The principal reasons for seeking consultation were because the pain was more frequent and severe ( $72 \%$ and $24 \%$, respectively). The mothers' main questions concerned the reasons for headache and reassurance about their fears (respectively $61 \%$ and $46 \%$ ). Fiftysix percent of the mothers thought that the use of symptomatic drugs was necessary, while only $33 \%$ thought that preventive drugs were useful and $34 \%$ of mothers feared that they cause dependency or serious side effects. The children wanted to be mainly reassured $(60 \%)$, know the reasons for the headache $(45 \%)$, and if they would suffer from headaches in adulthood (36\%) (mothers and doctors only $4 \%)$. The children thought that the use of symptomatic drugs was necessary $(68 \%)$ and that preventive therapy was useful $(61 \%)$. Fifty-eight percent of doctors believed that the principal reasons for the consultation were the frequency $(58 \%)$ and intensity $(26 \%)$ of headache, and the fear of tumour $(24 \%)$. In the opinion of the doctors, the more important mothers' questions concerned reassurance about their fears and the prescription of medical diagnostic tests (60\% and 50\%, respectively). They believed that the children wanted to be mainly reassured, cured and to have explanations about the headache $(62 \%, 38 \%$ and $44 \%$, respectively). The paediatricians $(90 \%)$ believed that symptomatic drugs were necessary and that preventive therapies were useful $(74 \%)$.

Discussion These data suggest that the child's and mother's main concerns pertain to explanation of headache and reassurance about it, while the prescriptions of drugs or medical tests are not believed necessary. The doctors' opinions seem in part similar. The main differences regarded the use of diagnostic tests and pharmacological therapies and the question about headache progression into adulthood made by children.

\section{HEADACHE IN CHILDHOOD AND ADOLESCENCE II}

\section{DEPRESSION AND CHRONIC HEADACHE IN CHILDHOOD AND ADOLESCENCE}

M.A. Tavoni, O. Papa, E. Cesaroni, C. Cardinali

Riuniti University Hospitals, Paediatric Neurology Department, Children’s Hospital “G. Salesi”, Ancona, Italy; e-mail: npisalesi@ ao-salesi.marche.it

Introduction The role and importance of the psychiatric and psychological aspects in determining and influencing the insurgence and evolution of chronic headache in childhood and adolescence have been the object of numerous studies. Many authors have tried to analyse the possible correlations between depression, anxiety disorders and headache. Objective The aim of our study was to analyse the possible correlations between chronic headache and depressive disorder with or without anxiety disorder.

Materials and methods Sixty-two children (38 males, 24 female; mean age 9 years, 5 months; range: 6-13 years) were enrolled from 493 subjects seen from 2003 to 2005 among the outpatients from the Departments of Headache and Psychology of the "Ospedali Riuniti "G. Salesi" Children's Hospital. Fifty-two patients affected with chronic headache were subdivided into 3 subgroups: chronic tension-type headache $(\mathrm{CTTH})$, chronic daily headache $(\mathrm{CDH})$ and mixed headache (CTTH and headache); 10 patients affected from episodic tension-type headache (ETTH) were identified according to IHCD-II 2004 criteria. The patients under investigation were evaluated by the juvenile headache flow-chart (haematological, clinicians, neurophysiologic and neuroimaging exams) and by a psychodiagnostic protocol that we use in our centre.

Results Following the DSM IV criteria the subjects of the two groups were subdivided in: 
- Depressive Disorder Mood (DDM);

- Depressive Disorder Mood + generalized anxiety disorder (DDM+GAD);

- Depressive Disorder Mood + Learning Disorder Not Otherwise Specified (DDM+LDNOS);

- Depressive Disorder Mood + Oppositional Defiant Disorder (DDM+ODD);

- Generalized Anxiety Disorder (GAD); Others.

The clinical history of the 62 subjects was negative for psychiatric pathologies or history of acute traumatic events. Depressive disorders were comorbid in $37 / 52$ of the subjects with chronic headache, primarily represented by DDM + GAD (30/52). The GAD subgroup was present in 13/52 chronic headache patients and in 3/10 ETTH patients. Male gender was more represented, but female gender prevailed in the group of chronic headache with DDM + GAD. Chronic headache was not associated with DDM + ODD but was present in 2 patients affected by ETTH. The prevalence of depressive disorders was greater in chronic headache $(71.1 \%)$ than in ETTH $(40 \%)$. DDM was more frequently associated with GAD $(57.6 \%)$.

Conclusions Chronic headache is frequently associated with depressive and anxiety disorders both in adult and paediatric patients. Headache can be the first symptom silently expressed by the depressed child.

Therefore, headache that evolves to acquire chronic characteristics must be carefully evaluated because it could mask important psychiatric disorders that should be considered for the diagnosis and therapy.

\section{PSYCHOLOGICAL ASSESSMENT OF MIGRAINOUS CHIL- DREN AND ADOLESCENTS}

${ }^{I} P$. Mariotti, ${ }^{I} R$. Chiera, ${ }^{l} F$. Perrino, ${ }^{I} S$. Staccioli, ${ }^{I} P$. De Rose, ${ }^{2}$ C. Vollono, ${ }^{2}$ G. Della Marca, ${ }^{3}$ G.M. Vecchio, ${ }^{4}$ C. Pastorelli

${ }^{1}$ Unit of Child Neuropsychiatry, and ${ }^{2}$ Unit of Neurology, Policlinic "A. Gemelli", Catholic University, Rome, Italy; ${ }^{3}$ Faculty of Psychology 2, and ${ }^{4}$ Department of Psychology, University of Rome, "La Sapienza", Rome, Italy; e-mail: pmariotti@rm.unicatt.it

Introduction General population studies suggest a non-casual association between headache, major depression and anxiety disorders [1]. The consensus is that migraine sufferers are at higher risk of depression, bipolar disorders and certain forms of anxiety - this is particularly true for migraine with aura. Co-morbidity with psychiatric disorders has also been described for chronic tension-type headache and for chronic daily headache [2]. The data available in the literature are still relatively scarce and contradictory, especially for young people.

Objective The aim of our study was to evaluate the association between headache and different psychological characteristics, including tendencies toward depression, perfectionism and repressed aggression, in children and adolescents.

Methods Clinically referred children and adolescents $(n=45)$ 8-18 years of age, subdivided into two age groups: $8-10$ years $(n=19)$ and $11-18$ years $(n=26)$, suffering from primary headache according to the diagnostic criteria of the International Headache Society, 16 with migraine with aura (MA), 29 with migraine without aura (MO), and 1021 normal controls without headache (NC), were assessed using the Parent Child Behaviour Checklist (CBCL), the Youth Self Report (YSR), Children's Depression Inventory (CDI), and the Centre for Epidemiological Studies Depression Scales (CESD).

Results According to the literature, our data confirm that children with migraine have significantly higher levels of total internalizing, particularly for somatic complaints with respect to those without headache. Attention has been focused on the YSR interview, a self-report assessment, never used until now in this field, that was administered to adolescents aged 11-18 years. Both thought problems and internalizing symptoms in migraineurs have been demonstrated by YSR. We wish to emphasize that thought problems are not significantly different between the general paediatric population and migraineurs in some studies in parental reports (CBCL). Moreover, CDI (self rating of depression) results show statistically significant differences between migraine and non-headache paediatric subjects, in comparison with other studies published in the literature.

Conclusions We stress the importance of the use of YSR for the assessment of psychological traits in migrainous children and adolescents, because generally parents have been found to be poorer at internalizing symptoms than the child, and parents' reports of CBCL may underestimate anxiety symptoms. This holds not only for the diagnostic and therapeutic implications but also for the prognostic aspects.

References

1. Radat F, Swendsen J (2004) Psychiatric comorbidity in migraine: a review. Cephalalgia 25:165-178

2. Oedegaard KJ, Neckelmann D, Mykletun A et al (2006) Migraine with and without aura: association with depression and anxiety disorder in a population-based study. The HUNT Study. Cephalalgia 26:1-6

\section{EFFICACY OF TOPIRAMATE IN PAEDIATRIC MIGRAINE PROPHYLAXIS}

${ }^{1}$ E. Conicella, ${ }^{2} \mathrm{~F}$. Vigevano, ${ }^{2} \mathrm{M}$. Valeriani

${ }^{I}$ DEA and ${ }^{2}$ Headache Centre, Division of Neurology, "Bambino Gesù" Paediatric Hospital, IRCCS, Rome, Italy; e-mail: m.valeriani@tiscali.it

Introduction Migraine is the most common type of primary headache in childhood and often hampers common daily routines.

Objective The aim of our study was to investigate the efficacy and safety of topiramate in the prevention of paediatric migraine.

Materials and methods Twenty-five children (mean age 11 years, range 5-16 years; 10 females, 15 males) were recruited. In 23 children, migraine without aura was diagnosed according to the International Headache Society classification (ICHD-II, 2004), while the remaining 2 children had migraine with aura.

Results Before treatment, our patients showed $8 \pm 5.7$ (mean \pm standard deviation) pain attacks per month. The children received a mean topiramate dose of $1.3 \mathrm{mg} / \mathrm{kg} /$ day, ranging from 0.5 to 1.8 $\mathrm{mg} / \mathrm{kg} / \mathrm{day}$, for a period of $3.2 \pm 1.2$ months. Treatment was effective (reduction in attack number $\geq 50 \%$ ) in 19 out of 25 children $(76 \%$ ). In particular, 3 patients (12\%) became free from migraine episodes. One patient $(4 \%)$ interrupted the treatment for adverse side effects (excessive drowsiness). Seven patients in which the initial treatment was effective underwent a worsening in migraine frequency after topiramate interruption and needed a further treatment after $4.3 \pm 2.8$ months. Six patients had a reduction in the frequency of pain episodes greater than $75 \%$, while in one child the therapy was ineffective. Two patients had a third treatment at 3 and 4 months, respectively, after interrupting the second topiramate cycle. The therapy was ineffective in both patients. Adverse events were observed in 6 out of 25 children (24\%). They consisted of drowsiness (3 patients), anxiety (1 patient), weight loss ( 2 patients), and behavioural disturbances ( 3 patients).

Discussion and conclusions Our results suggest that topiramate is a useful and safe tool in paediatric migraine prophylaxis. Even low doses allowed us to control migraine in most of our paediatric patients, thus improving their quality of life.

\section{MELATONIN, 3 MG, IS EFFECTIVE FOR MIGRAINE PRE- VENTION ALSO IN SCHOOL-AGED PATIENTS}

M. Carotenuto, F. Tagliente, F. Ruju, A. Pascotto

Department of Developmental Neurology and Psychiatry, Second University of Naples, Naples, Italy; e-mail: marco.carotenuto@unina2.it

Background Hypothalamic dysfunction may be present in migraine and in circadian periodicity of headache attacks, more specifically, the 
suprachiasmatic nucleus and melatonin have been suggested to play a role in the pathogenesis [1]. Altered melatonin levels have been found in cluster headache, migraine with and without aura, menstrual migraine, and chronic migraine. Melatonin may have many metabolic effects, such as anti-inflammatory effects (melatonin and indomethacin share similar chemical structure), reducing the up-regulation of proinflammatory cytokines, and inhibiting nitric oxide synthase activity and dopamine release. Melatonin administration is thus a possible candidate for migraine prevention [2].

Objective The aim of this open trial was to test the hypothesis of the potential effectiveness of melatonin for migraine prophylaxis also in school-aged children.

Patients and methods We performed an open-label trial of melatonin, $3 \mathrm{mg}$, for migraine prevention. Twenty-three patients (11 M, 12 F) aged 6-15 years (mean 12.8 years, $\mathrm{SD} \pm 1.38$ ) with episodic migraine with or without aura according to ICHD-II 2004 criteria, were screened for the baseline period. All patients started prophylactic treatment with melatonin, $3 \mathrm{mg}, 10$ minutes before bedtime for 3 months. A complete clinical interview and examination as well neurophysiological recordings (wake and sleep EEG) were performed for each patient. Study participants experienced between two and six attacks per month. Exclusion criteria included the presence of sleep disorders, as confirmed by the mothers of all subjects who filled out the Sleep Disturbances Scale for Children [SDSC]. The SDSC is a sleep questionnaire that consists of 26 items subdivided into 6 sleep disorder subscales according to American Sleep Disorders Association criteria: DIMS (disorders in initiating and maintaining sleep), SDB (sleep disordered breathing), DA (disorders of arousal), SWTD (sleep-wake transition disorders), DES (disorders of excessive somnolence), SHY (sleep hyperhydrosis).

Results Twenty out of 23 patients completed the study $(86.95 \%)$. That patients $(85 \%, 17 / 20)$ who completed the study had at least a $50 \%$ reduction in intensity and frequency of headache attacks. Nobody reported an increase in headache frequency. Complete $(100 \%)$ response was achieved in 12 patients $(60 \%)$ and no adverse effects were recorded.

Conclusions There is increasing evidence to indicate that the hypothalamus plays a major role in the pathophysiology of several of the primary headache disorders, such as migraine, and hypnic headache $[1,2]$. Melatonin, an indole compound derived from serotonin, was reported safe and effective in adult patients [2], but no data were available for school-aged children. This is an original study to assess melatonin, $3 \mathrm{mg}$, for prophylactic treatment of headache also in childhood.

\section{References}

1. May A (2006) Functional anatomy of headache. Neurol Sci 27[Suppl 2]:S103-S106

2. Peres MF, Zukerman E, da Cunha Tanuri F et al (2004) Melatonin,

$3 \mathrm{mg}$, is effective for migraine prevention. Neurology 63:757

\section{EMERGENCY IN CHILDREN. BRAIN TUMOUR: CLINICAL CASES \\ D. Moscato, M.I. Peracchi \\ Headache Centre, Saint Charles Hospital, IDI Rome, Rome, Italy; e-mail:dmoscat@tin.it}

Brain tumours in children, due to their characteristic of slow invasiveness and easy infiltration in the nervous tissue, tend to give specific symptoms only when they are of a large size.

We present a few cases which came to our attention last year.

Martina is an eight-year-old girl, who came to us due to widespread headache that started three years before. She was a child presenting a conflicting separation since the age of 3 , which she responded to initially with hyporexia; she was anxious, agitated, very attached to her mother. The symptoms seemed linked more to the psychological aspects. Neurological testing was negative. During Day Hospital she presented an intense headache crisis, which receded without treatment with NSAIDs. A MRI identified a new formation in the posterior cranial fossa. Surgical intervention revealed an adenoma, 2 months later, the headache had fully disappeared.

Giorgia is 13-years old, suffering from migraine for two years. Her parents are separated, and she is very attached to her mother, who still sleeps with her. In the past 3 months, she has suffered from sleep disturbances, sleep talking and frequent accentuated awakenings, with intense headache almost daily during the same period. Vomiting with coughing was present, but the girl had been following a diet for one month. The neurological examination was negative. Emotional tension was evident in the family. During hospitalisation she experienced slight difficulty in climbing the stairs. TAC shows a $6 \mathrm{~cm}$ hypodense formation in the temporal occipital area. Surgical resection of a grade 1 medulloblastoma was followed by one cycle of chemotherapy.

Gianluca is 5-years old, and had been complaining of intense cluster headache for a few months after occipital cranial trauma without loss of consciousness. The headache worsened overtime with an increase in severity. During Day Hospital, 10 days after the trauma, he complained of diffuse violent headache, with crying. Neurological examination was negative except for slight fluctuations on Romberg. CT showed a voluminous new formation in the posterior cranial fossa. He was transferred to the Neurosurgery Unit, where neurological signs began with vomiting, drowsiness, dysmetria, and dysarthria. Surgical resection of a Grade 4 medulloblastoma was followed by chemotherapy and radiotherapy. The following year he relapsed, and was operated on again, followed by another cycle of chemotherapy, but his condition worsened. Sixteen months after being diagnosed, he died.

Conclusions In the cases presented above the specific symptoms of intracranial pressure, vomiting and papilledema stasis without headache, as well as those linked to focality, are absent. Also, the neurological examination yielded no precise indications. The same psychological characteristics could have led to the wrong diagnosis. Observation overtime of the reactions of children to headache during Day Hospital had led us to select neuroimaging testing, which led to the diagnosis. Therefore, we believe that a child with an important headache must always be visited during a crisis.

\section{HEADACHE IN A PAEDIATRIC EMERGENCY DEPARTMENT}

${ }^{1,2}$ L. Calistri, ${ }^{2}$ M. Guasti, ${ }^{1,2}$ C. Scalas, ${ }^{2} M$. de Luca, ${ }^{3} F$. Festini, ${ }^{2}$ S. Masi, ${ }^{2}$ F. Mannelli

${ }^{1}$ Headache Centre for Childhood and Adolescence, Paediatric Neurological Clinic, ${ }^{2}$ Emergency Department, ${ }^{3}$ Department of Paediatrics, University Hospital “A. Meyer”, Florence, Italy; e-mail: l.calistri@meyer.it

Objective The aim of this study was to determine the prevalence of headache as the chief complaint in patients presenting to a paediatric Emergency Department (ED), to assess its characteristics, to study the spectrum of primary and secondary headache diagnoses and to analyze the diagnostic tests and the treatment applied.

Methods We retrospectively analysed the clinical records of patients presenting to the ED of the Paediatric Hospital A. Meyer of Florence, Italy with the chief complaint of headache from January 1, 2005, to June 30, 2005. All the patients underwent a clinical evaluation/case history, and if necessary also had laboratory or imaging tests performed. The ED records were searched for triage color code, age, gender, location of pain, associated symptoms, case history, physical examination (particularly neurological examination), assessments and specialists' visits, therapy at the ED, time spent at the ED, and outcome (discharge - hospitalization - further assessments at the Centre for Headache in Childhood and Adolescence).

Nosology was established using the International Classification for Headache Disorders ICDH-II. 
Results Two hundred and twenty-three clinical records (2.2\% of the total number of visits) concerning 203 patients (121 male and 82 female) were analyzed; the average age was 8.9 years (range 2.1-17.1 years). Primary headaches (migraine and tension-type headache) were recorded in 33 subjects (16.2\%): 10 patients with migraine without aura, 10 subjects with probable migraine, 2 cases of migraine with aura, 2 subjects with benign paroxysmal vertigo of childhood; from all the tension-type headaches, 7 were frequent, 4 infrequent, and 1 chronic. Three subjects were found to have both migraine and tension headache (mixed headache). Secondary headaches were mainly attributed to infections in 80 subjects $(39.4 \%)$ and to sinusitis in 16 patients $(7.9 \%)$. Five cases $(2.5 \%)$ were found to be attributed to serious neurological conditions: 1 cerebral empyema, 1 cerebral ischemia, 1 intracranial haemorrhage attributed to arteriovenous malformation, and 2 patients with intracranial hypertension already treated at the Oncohematology Centre for cerebral expansive lesion. After the clinical assessment/case history, 144 patients $(64.6 \%)$ were discharged from the ED without further tests. Radiological and laboratory assessments and/or specialists' examinations were performed in $35.4 \%$ of the patients. Neuroimaging tests were performed in 27 patients of whom $44.4 \%$ presented pathological results. Eighteen subjects (8.8\%) were hospitalized.

Conclusions This study confirms that headaches in the paediatric ED have different etiology. In 57 subjects $(28.1 \%)$ headache could not be classified. Future prospective studies may be useful to assess the follow-up in this group of patients.

\section{HEMORRHAGIC PROLACTINOMA IN THE PAEDIATRIC AGE GROUP: TWO CASES WITH DIFFERENT CLINICAL PRE- SENTATION}

${ }^{l}$ P.A. Battistella, ${ }^{2}$ G.B. Pozzan, ${ }^{I}$ A. Permunian, ${ }^{1}$ I. Toldo, ${ }^{l}$ S. Sartori, ${ }^{3} R$. Faggin

${ }^{1}$ Department of Paediatrics, University of Padua, Padua, Italy; ${ }^{2}$ Paediatrics Unit, Hospital of Mestre, Mestre, Venice, Italy; ${ }^{3}$ Service of Paediatric Neuro-Surgery, University of Padua, Padua, Italy; e-mail: battist@pediatria.unipd.it

Introduction Pituitary juvenile adenomas are rare (2\% to $6 \%$ of all supratentorial neoplasms) and prolactinoma is the prevalent tumour [1]. Subjects and methods We present two paediatric cases of hemorrhagic prolactinoma. The first case was a 7 -year-old boy, with an acute left-sided visual loss (acuity 1/10 and fundus oculi was normal). Visual evoked potentials (VEPs) showed increased latencies after left eye stimulation. MRI showed a suprasellar lesion hyperintense on T1- and T2-weighted images without enhancement and bordering on the optic chiasm. The serum prolactin was $439 \mu \mathrm{g} / \mathrm{L}$ (normal $<15)$, with decreased cortisol $(88 \mathrm{nmol} / \mathrm{L}$, normal $>198)$ and ACTH $(3 \mathrm{ng} / \mathrm{L}$, normal $>10)$. After steroidal therapy, visual acuity improved (8-9/10). He then underwent a transphenoidal complete resection of the prolactinoma and subsequently presented panhypopituitarism. The second case was a 17-year-old girl with a three-month last recurrent, moderate, biparietal headache associated with photophobia, especially in the afternoon. In the previous year an oligomenorrhea was treated with hormonal therapy. This treatment was stopped because of headache, which was unresponsive to pill withdrawal. Neurological examination was normal. MRI showed a T1and T2-weighted hyperintense suprasellar lesion without enhancement. As in the first case, these findings indicated a hemorrhagic pituitary macroadenoma. The serum prolactin was $203 \mu \mathrm{g} / \mathrm{L}$ (normal $<25$ ). Visual acuity and visual field were normal. After the surgical resection, she needed substitutive hormonal therapy and, since then, she has never suffered from headache.

Discussion and conclusions Hemorrhagic prolactinomas are rare in children [2]. Patients usually present headache and visual field deficits. Other findings are gynecomastia and puberal delay in males and amen- orrhea in females. The cases reported are characterized by peculiar clinical features. The first one presented a pituitary apoplexy, rare in paediatric age group, and a monolateral visual loss with VEPs abnormalities consistent with an optic neuritis. In the second one, instead, the oligomenorrhea although of a mild pattern was the key-sign for the correct diagnosis of a secondary headache.

References

1. Arginteanu MS, Post KD (2001) Pediatric pituitary tumors. In: Keating RF Goodrich JT and Packer RJ (eds) Tumors of the pediatric central nervous system. Thieme, New York, NY, pp 292-301

2. Sugita S, Hirohata M, Tokutomi T et al (1995) A case of pituitary apoplexy in a child. Surg Neurol 43:154-157

\section{SPHENOID MUCOCELE IN CHILDREN: DESCRIPTION OF TWO CASES}

P.A. Battistella, L. Bianchin, S. Sartori, A.M. Laverda, R. Faggin, I. Toldo, P. Drigo

Department of Paediatrics, University of Padua, Padua, Italy; e-mail: battist@pediatria.unipd.it

Introduction Sphenoid mucoceles are very rare, benign cystic lesions (1\% of all localizations) $[1,2]$. Due to connection with several structures (cavernous sinuses, pituitary, orbits and choanas), clinical manifestations can be extremely variable.

Subjects and methods Case 1: An 8-year-old male presented with a subcontinuous mild-moderate, bifrontal, pressing headache on awakening for seven days, without any associated symptoms. On the eighth day, an acute left III cranial nerve palsy with exotropia, ptosis and mydriasis appeared. CT scan showed an erosion of the parasellar structures and MRI revealed a lesion of the sphenoid body, hyperintense on both T1-T2 sequences without enhancement. The maxillofacial CT scan confirmed the sphenoid body lesion. Hormone levels, visual fields and acuity were normal. After ten days the headache spontaneously disappeared, but nerve deficit persisted. Endoscopic trans-sphenoidal permitted total removal of the lesion, followed by histological confirmation and complete resolution of the third cranial nerve palsy. He was asymptomatic at the 5-year follow-up.

Case 2: A 12-year-old male since last year suffered from recurrent, pulsating, weekly headache, localized to the right frontal-ocular region, with photophobia and phonophobia, sometimes nausea, rarely vomiting and responsive to NSAIDs. His father suffered from migraine without aura. During the recent admission to our hospital the neurological examination was negative. Brain MRI showed hypointense signal on $\mathrm{T} 1$ and hyperintense signal on T2 in the left part of the sphenoid sinus, with partial involvement of the ipsilateral ethmoid and maxillary sinuses. Sinus CT confirmed a left-sphenoidal mucocele, with indication for an endoscopic sphenoidotomy.

Discussion and conclusions The first case presented a painful ophthalmoplegia which, considering only the clinical picture, could be diagnosed as ophthalmoplegic migraine (ICHD-II, 2004 classification, point 13.17), a very rare condition in young patients, or as Tolosa-Hunt syndrome (ICHD-II, 2004, point 13.16), which also presents with similar features, although with unilateral pain. Neuroimaging, however, documented a rare benign pathology.

In the second case, the patient may be affected by migraine without aura, considering the family history, the type and stability of attacks over time, the resolution with vomiting after the attacks, and the lateralization of the sphenoid lesion, contralateral to the pain. Thus the discovery of a sphenoid mucocele at an early stage can be considered accidental, even if this diagnosis is confirmed after surgery.

\section{References}

1. Prepageran N, Subramaniam KN, Krishnan GG, Raman R (2004) Ocular presentation of sphenoid mucocele. Orbit 23:45-47

2. Moriyama H, Nakajima T, Honda Y (1992) Studies on mucocoeles of the ethmoid and sphenoid sinuses: analysis of 47 cases. J Laryngol Otol 106:23-27 


\section{TWO CLINICAL CASES OF RED EAR SYNDROME (RES) CONNECTED WITH HEADHACHE}

C. Termine, C. Pintacrona, G.A. Magro, A. Vecchio

Multispecialty Paediatric University Department, Maternity Unit, AUSL 6, Child Neuropsychiatry Division, Palermo, Italy; e-mail: chiaratermine@virgilio.it

Introduction The Red Ear Syndrome (RES) is characterized by sudden events when the patient feels a kind of uneasiness (sometimes a real painful sensation) mainly localized in one ear, which becomes red and warm. Sometimes it happens in a spontaneus way, sometimes it is caused by triggers, like touching the ears, feeling warm or cold, moving the neck, chewing, drinking, coughing or sneezing, The first description of RES dates back to 1996 thanks to Lance [1] who studied its characteristics in 12 patients, some of them having a past of headache. More recently, Raieli et al. 2002) [2] discovered a closer connection between RES and migraine in eight patients. Here, we present two clinical cases that might confirm what is reported in the recent literature. The first one concerns a little boy of 11 years affected by an almost daily headache bilaterally localized to the forehead, causing heaviness of variable intensity lasting about 30 minutes together with nausea, vertigo, phosphenes, pallor, perspiration and hyporexia. The second case concerns a little boy of 7 years who was observed because of a symptomatology of weekly headaches, localized on the parietotemporal side, characterized by pulsating, variable intensity, and lasting no more than 30 minutes. In both cases, the headaches were accompanied by the reddening of a single ear.

Materials and methods Before admittance a careful clinical history was taken according to our protocol for headache diagnosis, and through an accurate clinical and instrumental neurological examination, following the guidelines of the Italian Headache Society for the Study of Headache (SISC).

Results This diagnostic approach allowed us to place the patients' symptomatology in a context of headache connected with RES, bypassing the difficulty in classifying the kind of headache they were affected by, as often happens in patients of that age.

Conclusions Our experience is further proof of the observation made by other authors regarding the connection between RES and headache symptomatology. This suggests the possibility that RES, when present in patients suffering from headache, is epidemiologically understated at present, and the debate is still open on the possibility of including RES in an unclassified category of headache disorders according to the IHS classification.

\section{References}

1. Lance JW (1996) The red ear syndrome. Neurology 47:617-620

2. Raieli V, Monastero R, Santangelo G et al (2002) Red ear syndrome and migraine: report of eight cases. Headache 42:147-151 